\title{
Walking with perturbations: a guide for biped humans and robots
}

Jacques Duysens ${ }^{1,2} \mathrm{MD}, \mathrm{PhD}$ and Arturo Forner-Cordero ${ }^{1, *} \mathrm{PhD}$

1 Biomechatronics Lab., Mechatronics Department

Escola Politécnica da Universidade de São Paulo.

Av. Prof. Mello Moraes, 2231 - Cidade Universitária 05508-030 - São Paulo-SP - Brasil

Tel. +55 (11) 3091 9965/6011 (office/lab)

*Corresponding Author email: aforner@usp.br; arturoforner@gmail.com.

2 Dept. Of Kinesiology, FaBeR, Katholieke Universiteit Leuven. Belgium

Jacques.Duysens@faber.kuleuven.be 


\section{Summary}

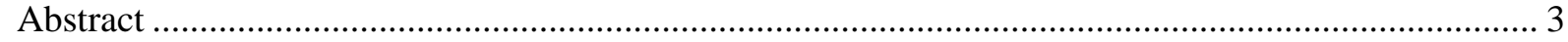

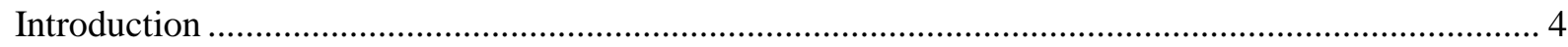

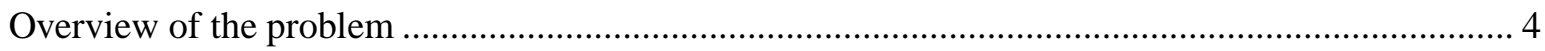

Generation of the biped gait patterns during unperturbed gait ........................................................... 5

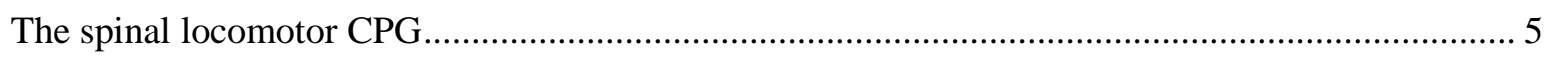

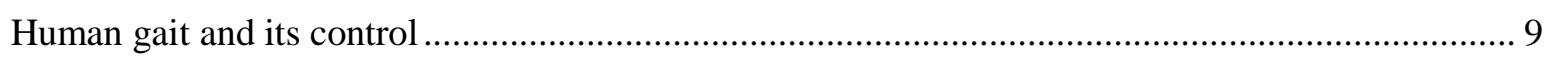

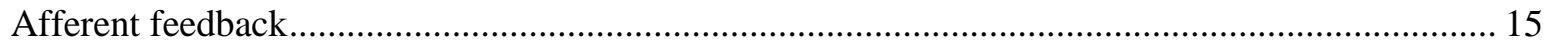

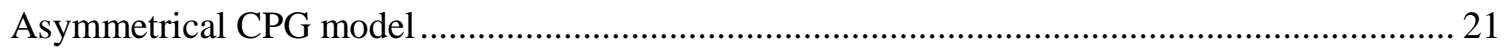

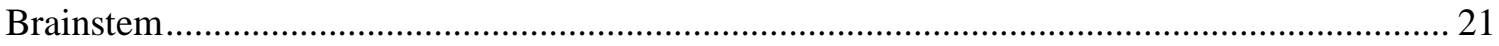

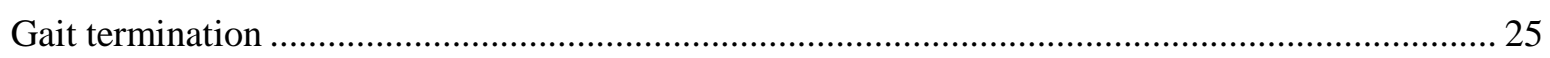

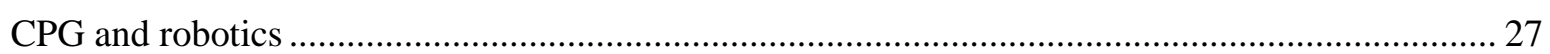

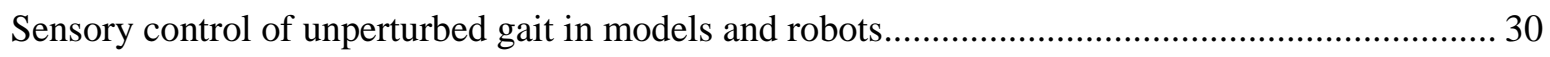

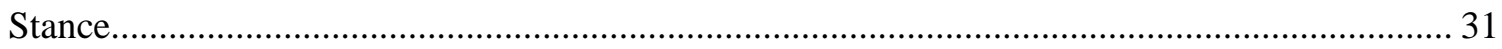

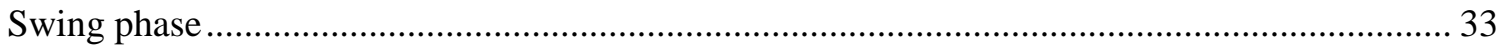

Phase-dependent modulation in models and robots .................................................................. 35

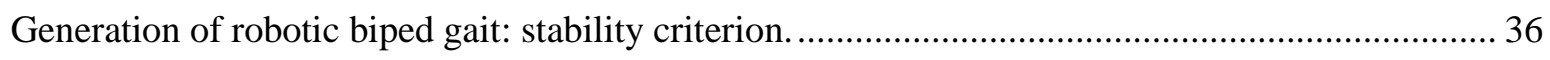

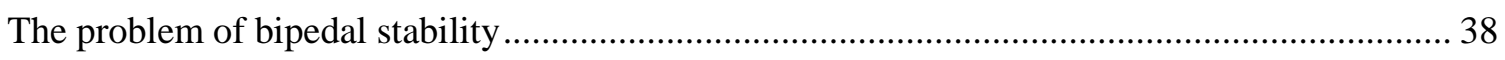

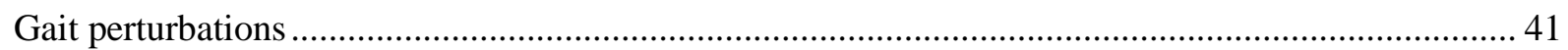

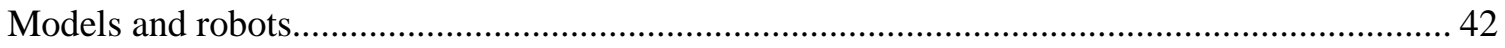

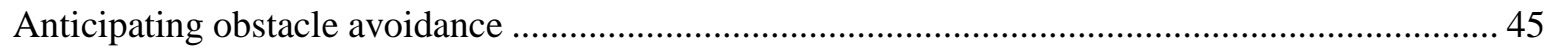

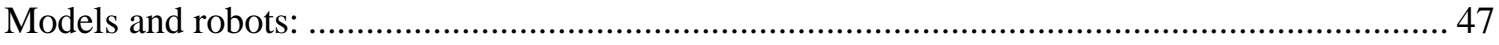

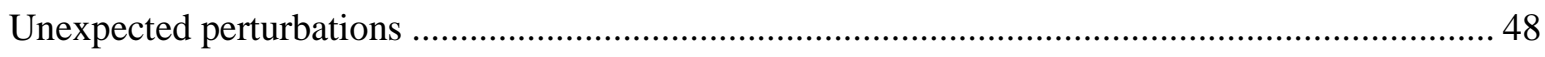

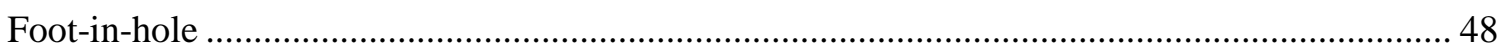

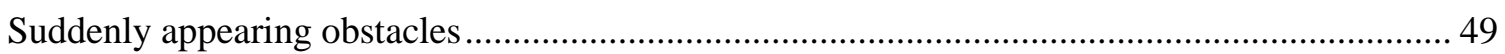

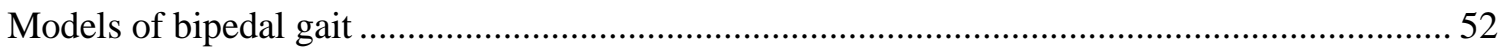

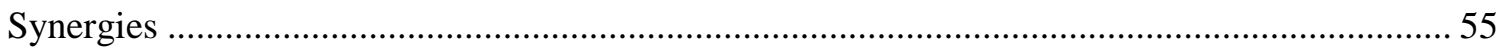

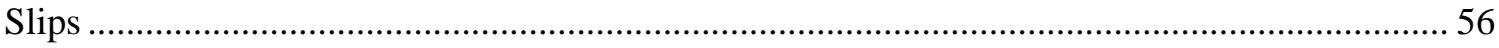

Recovery responses from gait perturbations in robots; a question of stability ................................ 60

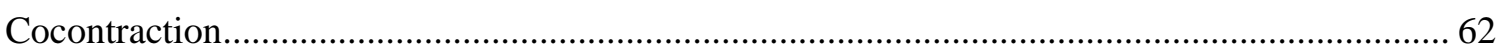

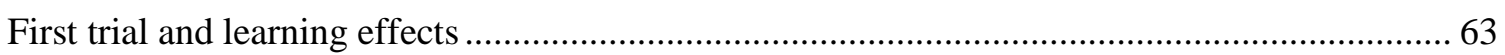

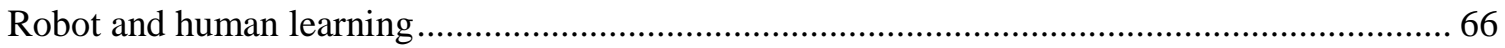

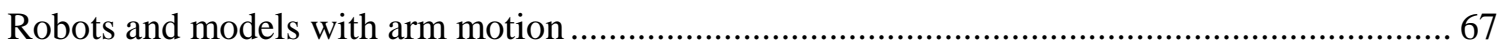

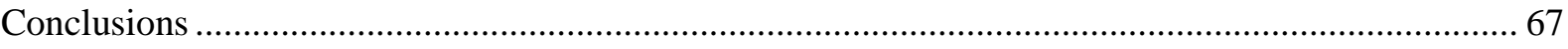

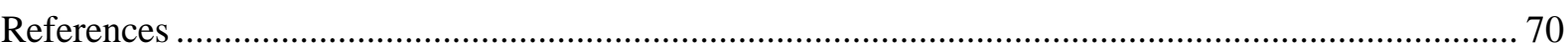




\section{Abstract}

This paper provides an update on the neural control of bipedal walking in relation to bioinspired models and robots. It is argued that most current models or robots are based on the construct of a symmetrical CPG (central pattern generator). However, new evidence suggests that CPG functioning is basically asymmetrical with its flexor half linked more tightly to the rhythm generator. The stability of bipedal gait, which is an important problem for robots and biological systems, is also addressed. While it is not possible to determine how biological biped systems guarantee stability, robot solutions can be useful to propose new hypothesis for biology. In the second part of this review, the focus is on gait perturbations, which is an important topic in robotics in view of the frequent falls of robots when faced with perturbations. From the human physiology it is known that the initial reaction often consists of a brief interruption followed by an adequate response. For instance, the successful recovery from a trip is achieved using some basic reactions (termed elevating and lowering strategies), that depend on the phase of the step cycle of the trip occurrence. Reactions to stepping unexpectedly in a hole depend on comparing expected and real feedback. Implementation of these ideas in models and robotics starts to emerge, with the most advanced robots being able to learn how to fall safely and how to deal with complicated disturbances such as provided by walking on a split-belt. 


\section{Introduction}

\section{Overview of the problem}

Often, the field of artificial generation of biped gaits, the gait synthesis both in virtual and physical robots, is inspired by ideas from biology [1]. However, these ideas change over time and therefore it is essential to update the knowledge and provide new insights. This is the first aim of the current review. In particular, major advances were made thanks to new techniques. For, example, optogenetics allows for the selective activation of cell populations. These methods are based on a combination of genetics and optics and can be used in the behaving (walking animal) by delivering light into selected locations in the brain. The basis of the method in the use of light-activated proteins (opsins) inserted in viruses. Such genetically engineered fluorescent proteins change their fluorescence when bound to a given neurotransmitter such as glutamate. While biology inspires robotics, the reverse is not so obvious. However, robots can implement biologically plausible stability criteria and serve as physical models to validate the hypotheses about biological motor control. A third aim is to extend the field to include gait perturbations, while previous reviews focused on steady state gait. It is important to note that biology can inspire engineering design at several levels, related to the different levels of insight about the biological mechanisms, building on the distinction between biomimetism and bioinspiration [2]. Bioinspiration is defined by the ISO/TC266 as a design method based on the observation of biological systems [3]. This approach does not need to understand the goals and mechanisms of the biological system. On the other hand, the biomimetism does require a deep understanding of the biological system to solve a technological problem. The biomimetic approach uses models of biological systems in order to transfer these models to appropriate solutions. 
In previous reviews the work on insects was included as well [4-6] suggesting the application of insect locomotion to the design of robots [7]. Here this work is not discussed in detail since the focus is on data on mammals in relation to human or robot biped simulations. Some excellent reviews on "lessons from biology" are available $[6,8,9]$. However, an update is needed because of new insights and because of the inclusion of gait perturbations, an emerging area in robotics, as robots go from the lab to our daily lives and walk around in any environment.

Following this idea, the paper is structured as follows. In the first place we will review the new biological concepts that have inspired robotic walking such as the pattern generators. Afterwards, we will look at an aspect of robots that has served to understand human gait: the stability criteria proposed for biped robots. Once we have reviewed the normal gait we will look at perturbed gait, considering both expected and unexpected perturbations, and the different strategies to implement recovery.

\section{Generation of the biped gait patterns during unperturbed gait}

\section{The spinal locomotor CPG}

In order to understand the neural control of locomotion it pays to look back in evolution. As walking vertebrates, we evolved from primitive vertebrates such as the lamprey which use undulatory motions to swim $[10,11]$. These motions were caused by using a rostrocaudal wave of excitation along their spinal cord. Hence a first question arises: Is this rostrocaudal gradient preserved in mammals such as humans?

To answer this question, we can look at the next evolutionary step towards quadrupedal vertebrates, the development of appendages (fins or legs), which appeared first in the elasmobranchs (sharks and rays) some 420 million years ago [11]. The neural locomotion networks of the skate Leucoraja erinacea, a well-studied elasmobranch, were found to be similar 
to those of mammals. However, instead of legs, these animals moved fins in a forwardbackward motion with left-right alternation, that also allows locomotion on land [12]. It is indeed known that some fish can walk, and this strongly suggests that mammal CPG circuits were already basically present in these marine vertebrates. In this view the human arms and legs stem from the paired fins of primitive fish [13].

What remained from the rostrocaudal activations in further evolution? In walking vertebrates such as humans there is also evidence for rostrocaudal waves in experiments where gravity is relatively unimportant, such as in air-stepping [14]. Similarly, in ventral root recordings at thoracic levels in the isolated rodent spinal cord during fictive locomotion there is a similar wave-like sequence of motor neuron activation [15]. Other species show the same phenomenon, for example in the frog where a rostrocaudal traveling wave activates the synergies in the proper sequence for locomotion [16].

The rostrocaudal activation then had to be translated into motions of legs. The first force to be dealt with by these primitive legs was gravity in order to lift the legs from the ground. Therefore, flexor pattern generation emerged, presumably first by co-opting primitive swim circuits [17]. In the latter work on genetic manipulation of mice, the evolutionary primacy of Flexor Pattern Generation was demonstrated by the prevalence of flexor firing in FoxP1MND preparations, in which the identity of limb-innervating motor neurons was reverted to a state resembling that of motor neurons that direct undulatory swimming in primitive aquatic vertebrates. After reversion of motor neuron identity, almost all firing patterns became flexor like [17].

If flexor activity is the crucial element in locomotion one may expect that flexor centers are most closely situated to the main brain structures. Indeed, in the cat or mouse, the flexor centers for the hind legs are located rostrally (L1-L3) while the extensor centers are situated more caudally (L4-L6). The threshold for evoking rhythmic activity is always lower rostrally, 
suggesting a closer link with the rhythm generator structures. This is true for chemical activation [18-20] as well as for opto-genetic stimulation [21-23]. In the latter study [23], it was found that caudal stimulation yielded a lower frequency of rhythmic activity indicating a weak link to rhythm generation. In the neonatal rat the key structure for rhythm generation is clearly located in the rostral lumbar segment [18]. The emerging picture in the neonatal rodent is that the more rostral segments of the lumbar cord can drive the more caudal segments [24]. The rostrocaudal gradient for rhythm generation potential was also seen in studies on cat scratching, where the pattern-generating capacity of the anterior half of the hindlimb enlargement was found to be greater than the posterior half [25]. A similar asymmetric distribution of pattern-generating elements in the spinal cord has been described in the turtle $[26]$.

Where does extensor activity enter the stage? Here one needs to go back to the events that took place when fishes became land animals, from lobe-finned fish (sarcopterygians) to limbed vertebrates (tetrapods) [27]. Flexion of the limbs had to be complemented by extension to support the animal. Hence extensors had to be activated in relation to the load to be supported. Load receptors could provide the necessary signals, and these could provide reinforcing feedback to the extensors. It is striking that this organization seems to be present in all legged walking animals [4], including insects [28]. It seems likely that these events helped shape a neuronal extensor center, which could elicit synchronous extension of the whole limb. This extension is basically working with gravity at the end of swing, therefore there is less need to be activated by a rhythm generator. Once on the ground, the load feedback reinforces extension.

This leads to a basic asymmetry because the flexor center is much more tightly coupled to rhythm generator circuits than the extensor center. This fits nicely with the rostrocaudal wave of activation, described above. 
This new picture of the CPG was schematically summarized in Figure 1a, based on the model presented by Brownstone and Wilson in a study on neonatal mouse spinal cord [29].

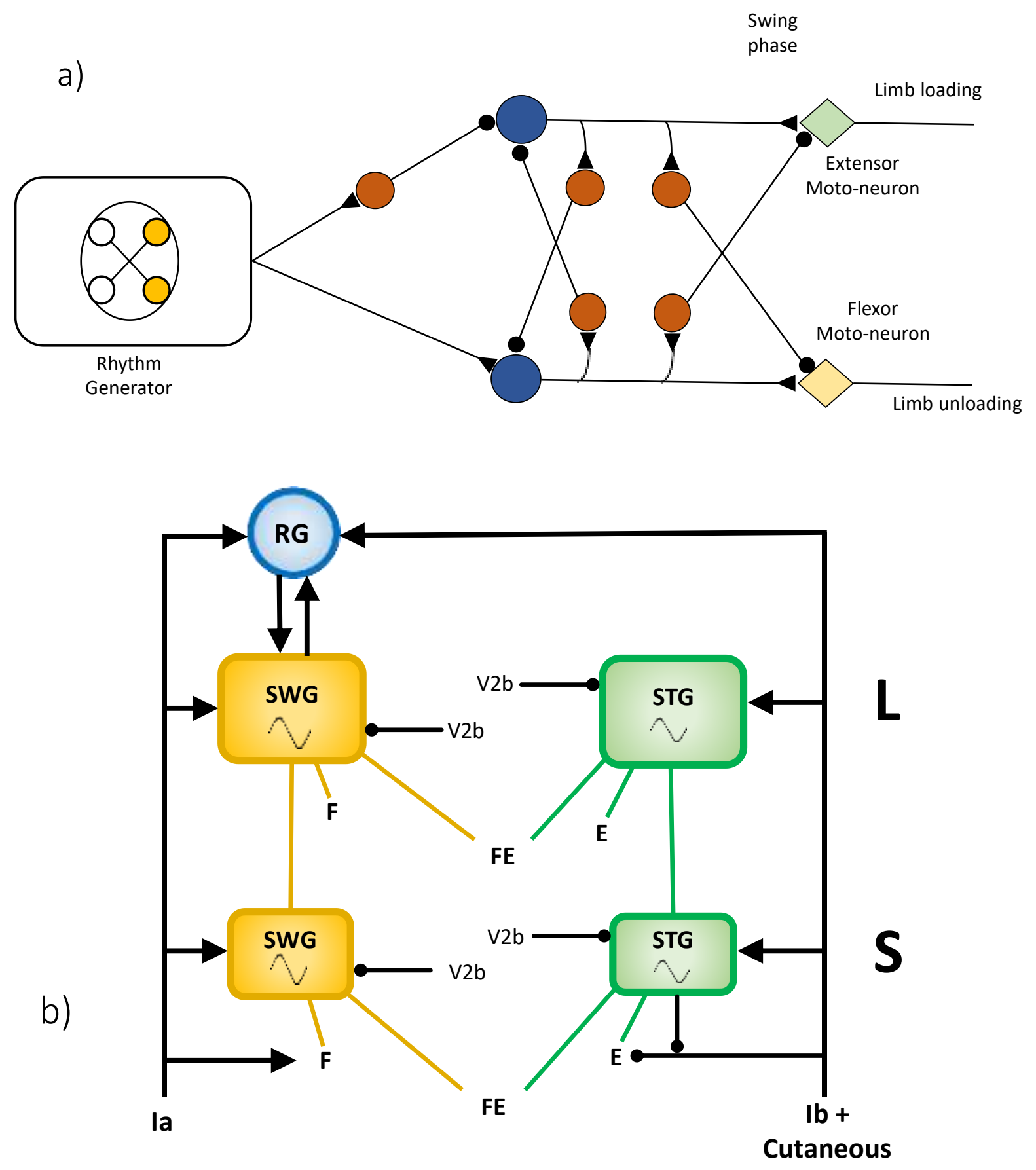

Figure 1. Central Pattern Generators of gait:

a) Model of an asymmetrical CPG as proposed for the neonatal mouse spinal cord (adapted from Brownstone and Wilson [29]). A rhythm generator is thought to send excitatory commands to a flexor 
half center while sending in parallel inhibition to the extensor half center. When the latter inhibition subsides, the extensor center is activated which leads to limb loading in the stance phase.

b) Model of the human CPG (as based on the model proposed by Ivanenko et al. [30]) containing a rhythm generator (RG) and CPG nodes for swing (SWG) and stance (STG), with lumbar (L) and sacral (S) levels. There are outputs for flexor (F), extensors (E) and biarticular muscles (FE). The Ia and $\mathrm{Ib}$ afferents are connected to the swing and stance half centers, respectively. The V2b indicate the role of this group of spinal interneurons. This human CPG model, proposed in [30] ,consist of two interconnected CPGs, a dominant lumbar (L) one and a second sacral (S) one. These CPGs consist each of two parts which are mutually inhibitory (push-pull). One half-center is the swing generator (SWG) the other the stance generator (STG). The swing generator controls mostly flexor (F) muscles, while the stance generator controls extensors (E). The lumbar CPG is connected to upper leg muscles and the sacral CPG is linked to the distal muscles. Biarticular muscles (FE) such as the hamstrings receive input from both. These CPGs control various muscle synergies. Simplified one can assume that in the step cycle there is a rostrocaudal sequence of activations, starting with the lumbar SWG. At onset swing the hip flexors such as Iliopsoas are active (L1-2; synergy 4 in [30]), while the swing phase is terminated by activity in hamstrings (synergy 5). The latter activity is also crucial for onset stance in conjunction with activity in tibialis anterior (TA) to decelerate the body (synergy 1). During stance the ankle extensors are needed as antigravity muscles and for propulsion (S1-2; synergy 2). Hence overall a rostro-caudal sequence is seen with the exception of the end swing TA burst. This exception is well-understood in human physiology since humans mostly land on the heel and require TA to brake the foot landing. Hence in humans there are 2 bursts in TA, one at onset swing and one at end swing: there is an initial burst needed to accelerate the foot upwards. In mid swing the leg acts as a pendulum without any need for added TA activation. In animals that have less pendulum swing the flexor activity occurs throughout the swing phase, like the cat, mouse or insects such as the stick insect $C$. morosus [31]. Figure adapted from the human CPG model of Ivanenko et al. [30].

Triangle-ended lines mean excitatory connections while circle-ended ones mean inhibitory synapses.

\section{Human gait and its control}

While it may be assumed that there are many similarities in the control of gait in mice and in man, it is important to include the differences as well. In human overground walking the rostrocaudal traveling wave is also present, however it is supplemented by two periods of motoneuron activation related to two modules involved in support and decelerations in the 
loading response in early stance [14,32]. This support module depends on load feedback while the deceleration module depends on cortical activation of tibialis anterior, which in humans is an important muscle for the smooth landing of the foot after heel strike. Hence overall a rostrocaudal sequence is seen with the exception of the end swing TA burst. This exception is wellunderstood in human physiology since humans mostly land on the heel and require TA to brake the foot landing. Hence in humans there are 2 bursts in TA, one at onset swing and one at end swing. These activations can be understood in terms of the biomechanics of the human leg. Given the weight of the leg there is an initial burst needed to accelerate the foot upwards. In mid swing the leg acts as a pendulum and there is no need for added activation of TA. In animals that have less pendulum swing the flexor activity occurs throughout the swing phase, for example in the cat or mouse but also in insects such as the stick insect $C$. morosus [31].

The swing stance sequence needs to be repeated, hence the coupling of the swing and stance centers to a rhythm generator, as could be borrowed from the swimming animals (fish and lamprey CPG). Not only the flexor centers are more closely related to rhythm generator circuitry than the extensor centers, they also may be relatively independent for different joints. In mice, for example, the individual flexor motor neuron pools can be recruited into bursting without any activity in other nearby flexor motor neuron pools [23]. This organization comes very close to the unit burst generator model proposed by Grillner [33]. In this model there are premotor centra for flexors and extensors for each joint of the leg. These centra can work independently or in a mutual inhibitory pair. It is still to be proven that independent bursting is functional during normal locomotion. It seems more likely that these "unit CPG synergist groups" can in turn be coupled to similar units at different joints thereby providing the possibility of recruitment in different orders (forward versus backward locomotion coordination). Grillner indicated that these units can possibly be further subdivided [33]. This fits well with the data of Hägglund that showed that the bursting could be evoked independently 
in flexors or extensors by excitatory input (activation of spinal glutaminergic cells) [23]. This seemingly contradicts the original half-center model which depends heavily on mutual inhibition between flexor and extensor centers. On the other hand, the bursting was observed in immobile animals while under natural locomotor conditions it appears that mutual inhibition of flexor and extensor motoneurons is indeed an essential feature. It was therefore suggested that the mouse CPG has a modular structure [23]. In this view the modules can be combined in different ways making it possible to support different gaits (alternating, in phase, backward, biarticular double bursting). In particular, gait speed seems to be organized in a modular fashion. This came to light with new techniques allowing identification or ablation of selective types of interneurons. For example, in mice the different types of gait (walk, trot, bound, gallop) were found to depend on different subpopulations of commissural interneurons [34].

For humans, as for quadrupedal animals, such as cats and mice, it seems likely that they also use a dominant flexor center, closely coupled to a rhythm generator but also linked to an opposing extensor center (Fig 1b). The mutual inhibition between flexor and extensor centers is the basis of one of the attractive CPG features, namely the "push-pull" mode of operation. An extensor center needs to be activated at times when the flexor center is inactive, which is readily achieved by having inhibitory connections between the flexor and extensor center. The activity of this extensor center could then be suppressed by the periodic activation of the flexor center (assisted by the rhythm generator) and driven by the decrease in load feedback that reinforces extension (see further). Flexion arises both from the activation from a flexor rhythm generator and from the release of suppression from the extensor center. Evidence for such "push-pull" operation of locomotor CPG was obtained by measurements in motoneurons during locomotor-like activity in the isolated neonatal mice lumbar spinal cord preparation $[35,36]$ or in immobilized decerebrate cats fictive locomotion [37-40]. In [35] it was noted that there was a marked asymmetry in the flexor and extensor drives. In particular, when CPGs were activated, 
the excitatory drive to extensors was much weaker than the one to the flexors. This arrangement makes sense in the view that the flexor centers need to be driven mostly centrally while extensor centra depend primarily on peripheral feedback [41-43]. All these features are summarized in figure $1 \mathrm{~b}$, which depicts the spinal locomotor circuitry in humans although it has many features borrowed mostly from rodent models (but not completely established for humans yet).

1) First, following the human CPG model of Ivanenko and colleagues [30], there are 2 CPG levels in figure $1 \mathrm{~b}$, one lumbar and one sacral, both controlling the legs. The first level controls mainly upper leg muscles, while the second one the lower leg muscles. According to cat studies, these 2 CPG levels are organized hierarchically and it is suggested that the L3-L5 segments are normally the "leading" ones, i.e., they determine the rhythm of activity in the whole spinal hindlimb center [25]. Furthermore, the organization is modular and the two parts of each CPG, namely the SWG and the STG, swing and stance generators, respectively, have the potential to be rhythmic autonomously. This follows from observations in the neonatal rat explored with optogenetic techniques [23]. The idea that there are several CPGs for each limb goes back to the idea of unit burst generators as explained above [33]. Each part of the CPG controls a set of muscles (either Flexors or Extensors) but a few muscles receive input from both, such as the biarticular muscles; see [44]. In Figure 1b the lumbar SWG has a larger symbol for oscillations to indicate that normally, according to the Rybak models, only flexor populations operate in the intrinsic bursting regime while the extensor populations are usually in a state of tonic activity, periodically inhibited by the flexor centers $[45,46]$. Nevertheless, under specific conditions, the STG can produce rhythmicity on its own, independently of the flexor rhythm [23]. It is presently unclear what role this extensor rhythm has in a functional context.

2) Second, the CPG's in figure $1 \mathrm{~b}$ are connected to a separated rhythm generator (for review see [47]). A separation of CPGs and rhythm generators was proposed because 
spontaneous deletions (failures) of activity bursts can be either resetting or not $[48,49]$. The lack of rhythm resetting during most of these deletions prompted the idea that these deletions were at the level of the pattern generator part of the CPG, not at the RG part [50]. On the basis of such evidence it was suggested by Rybak and colleagues and McCrea and Rybak that the spinal CPG has a two-level functional organization consisting of a rhythm generator (RG) and pattern formation $(\mathrm{PF})$ circuits $[49,50]$. With the new optogenetic methods available it was possible to identify at least one group of neurons involved in rhythm generation [51-53]. Indeed in the neonatal mouse spinal cord, glutaminergic $\mathrm{Hb} 9$ interneurons have been proposed to be part of the rhythm generator system (for review see [29,54]).3) In Figure 1b, the swing generators (SWG) are interconnected with a rhythm generator (RG), not to the stance generators (STG), leading to an asymmetrical configuration. These asymmetrical features are derived from various animal experiments. In premammillary cats walking on a treadmill, it was seen that spontaneous deletions occur much more frequently for extensors than for flexors, suggesting a weaker drive to extensors [55]. Flexor burst deletions are accompanied by tonic extensor activity while flexor bursting continues despite extensor burst deletions [42,55]. In fictive locomotion, the results were similar although occasionally there were exceptions (lack of tonic extensor activity with flexor deletions $[48,49])$. The mouse locomotion data further supported the picture since flexor and extensor deletions showed asymmetry [56]. While flexor deletions were accompanied by sustained ipsilateral extensor activity, the rhythmic flexor bursts continued without interruption during extensor deletions. This type of results supported the idea of an asymmetric CPG organization $[42,43,57]$. These finding led to models in which the flexor motoneurons receive stronger CPG input than the extensor ones (see e.g. Figure 8 in [35]). More generally the concept of asymmetry was part of other advanced CPG model $[45,47,56,58-$ 61]. It is of interest to note that the original idea for an asymmetric CPG, with dominance of flexor rhythmicity, actually came from studies on insects [41], For instance, Pearson and Iles 
(1970) studied the cockroach (Periplaneta Americana) locomotor system [62]. Discharges of coxal levator (flexor) motoneurons could be seen to remain rhythmic even in the case of deletions of extensor bursts $[62,63]$. This led to the idea of a flexor burst generator CPG with excitatory connections to flexors and suppressive effects on extensors [62,64]. From these observations and from those on deletions in the cat arose the general idea of an asymmetric CPG model [57], as further developed by Brownstone and Wilson [29] based on monosynaptically driven flexors and disynaptically inhibited extensors (figure 1a). In particular, the latter authors suggested that $\mathrm{Hb} 9$ interneurons in the neonatal mouse could be involved in an asymmetric model of locomotor rhythmogenesis through projections of electrotonically coupled rhythm-generating modules to flexor pattern formation half-centers.

4) In figure $1 \mathrm{~b}$ the symbol for intrinsic rhythmicity is larger for the swing generator than for the stance generator. This is based on the evidence that rhythmicity is always present in flexor parts of the CPG while the rhythmicity of extensor circuits is only present under some conditions (see [45-47]).

5) The lumbar CPG (larger in figure 1b) dominates the sacral one [24].

6) The SWG and STG are mutually inhibited, presumably through V2b interneurons. This is based on the evidence that alternating activity vanishes when these interneurons are inactivated (see [47] for review). Opto-genetic evidence also indicates that the mutual inhibition between flexors and extensors during locomotion relies on V2b interneurons in the neonatal mouse $[65,66]$. 


\section{Afferent feedback}

It has been emphasized that the CPG structures cannot be seen in isolation from sensory input since, under natural conditions, such CPGs work in close interaction with afferent activity during gait $[67,68]$.

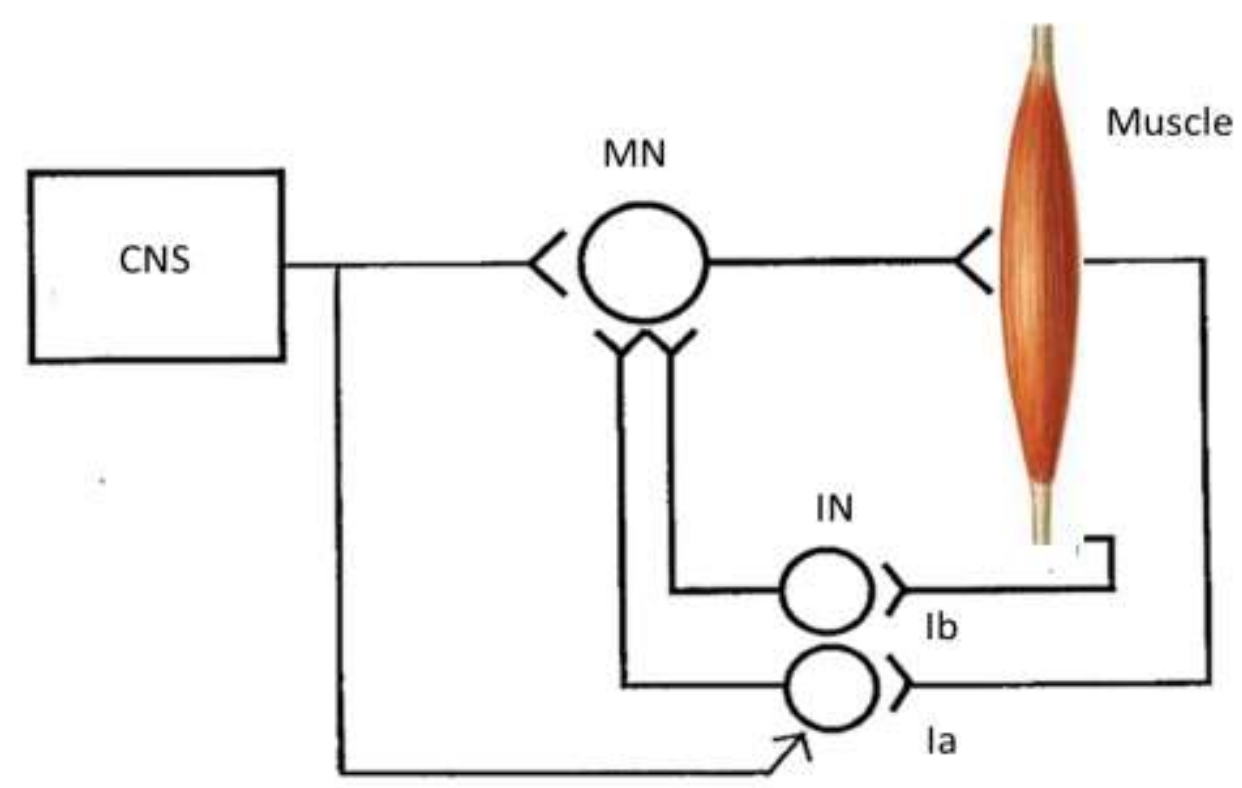

a) 
b)

Figure 2. Reflex and afferent information in the control of gait:

a) Gating of reflex pathways by the central nervous system (CNS). The central idea is that the CNS not only controls motoneurons (MN) and the connected muscles but also the afferent activity resulting from interaction with the environment (i.e. load receptors and Ib afferents from extensors, Ia afferents from hip flexors). This efferent control is schematically represented as input to interneurons (IN) although it is known that there are presynaptic interactions on the terminals of these afferents as well.

Figure 2b. Input/output of the CPG in relation to loading of the limb. During the gait stance phase, the load signals increase extensor activity and suppress the onset of the swing phase $[3,68]$. At the end of stance, the limb loading decreases and swing can be initiated. A second signal relates to hip angle [69]. At the end of stance, the hip extension is thought to assist the initiation of the swing phase using signals from the stretch of hip flexor muscles [70].

The interaction is bidirectional. Afferent activity modulates the CPG activity while the CPG funnels the afferent activity. These features are illustrated in figures $1 \mathrm{~b}$ and $2 \mathrm{a}, \mathrm{b}$ and will be discussed here in detail. Although the focus is on human physiology, we also refer to animal 
work, which was at the basis of some of the features (as previously described by Edwards and Prilutski [69] in their figure 9.9):

1) During stance, the load receptors input resulting from contact with the ground is, undoubtedly, the most important feedback (figure 2b). This is true for humans [70], as it is for the animal models $[4,68]$. In mammals, cutaneous receptors on the foot sole may be important as load receptors [41]; nevertheless, a main role is attributed to force sensors in muscles, the Golgi tendon organs with Ib afferents. Reflex pathways from the Ib afferents involve inhibitory connections in the resting state [71]. However, it was shown that the effects are excitatory during gait conditions [72]. These latter findings were confirmed by another group [73]. The switch from inhibitory to excitatory feedback is discussed below (see point 3)). In humans, some evidence for the suppression of inhibitory Ib actions during gait was obtained as well $[74,75]$.

2) An important feature presented in Figure $1 \mathrm{~b}$ is the Ib positive (reinforcing) feedback connection to the stance generator (STG). When there is extra loading during stance, the support phase needs to be prolonged and the onset of swing delayed. This was observed in the walking premammillary cat [72]. Later cat work demonstrated rhythm entrainment and resetting effects [76-79].

In humans some evidence is available as well. In infants, it was shown that sudden loading of the leg prolongs the stance phase and delays the next swing phase [80,81]. More continuous loading in adult humans had a similar effect [82]. Experiments with unloading during gait provided further evidence for facilitatory Ib effects to continue stance, a positive force feedback. In the study by Grey et al (2004) [83] a rapid plantar flexion perturbation was applied to the ankle during the stance phase. The EMG of the ankle extensor soleus began to decline approximately $52 \mathrm{~ms}$ after the unloading perturbation, even when an anesthetic nerve block eliminated cutaneous input. It was argued that load feedback from Ib afferents of the 
soleus was the most likely source of muscle activity during the stance phase of human walking [83].

3) So far, the CPG has been considered to be regulated by afferent input. However, this is a too simplistic view, as emphasized by Pearson in 2004 [67]. Indeed, the CPG affects afferent transmission (see output from STG inhibiting the inhibitory pathways of $\mathrm{Ib}$ and cutaneous afferents in figure $1 \mathrm{~b}$; figure $2 \mathrm{a}$ ). Hence the better proposal is that there is a bidirectional interaction between CPG and afferent input (also proposed by Cohen in 1992 [84]). This fits well with recent proposals about the feedforward action of the CPG in motor control models, for instance in [85], the CPGs generate oscillatory signals more to assist the sensory information decoding rather than to produce feedforward motor commands. At the end of the stance phase there is a facilitation of afferent input which induces the swing phase (figure 1b). Inversely, at end swing the sensory input related to limb loading is facilitated. These actions can explain the phase-dependent reversal seen both in cat $[41,86]$ and in man $[87,88]$. While the reversal is well-documented (see further) it is admitted that the connections shown in the figure are hypothetical and need to be confirmed. They are partly based on similar schemes of Büschges and Manira [68] and of Pearson and Ramirez [89].

In humans it is even more difficult to prove these connections, although the phenomenon of reflex reversal is well-documented $[87,88]$. For example, the stimulation of cutaneous input from the foot sole leads to flexion in early swing but leads to extensor reflexes and flexor suppression at end swing and onset stance[87,88]. The CPG has an element of feedforward control since the inhibition of one generator is followed by post-inhibitory rebound, a process which Sherrington in 1913 [90] described as "successive induction". In humans and cats, the release of inhibition of the STG presumably results in extensor EMG activity even prior to touchdown near the end of swing, in the so-called E1 phase, another convincing example of feedforward control $[6,91]$. 
In figure $1 \mathrm{~b}$ it is suggested that the STG part of the CPG inhibits the afferents. This is partly speculative but there is some indirect evidence both from cats and humans. From the cat work it is known that excitability in cutaneous afferents is phasically modulated during the step cycle during fictive locomotion, when only CPG action can explain the results [92]. In humans there is clear evidence for phase-depending modulation of cutaneous afferents but there is no proof that the CPG is involved $[87,88,93,94]$. For Ib afferents it is again the cat work that provided the best evidence for modulation by the CPG [95]. In spinal cats injected with nialamide and L-DOPA, it was possible to evoke spontaneous fictive locomotion, during which stimulation of ankle and knee extensor group I afferents evoked oligosynaptic excitation of extensor motoneurons, rather than the "classical" Ib inhibition [96]. Similar results were obtained using clonidine to evoke fictive locomotion [97]. In humans it was shown that Ib inhibitory reflexes are suppressed during the stance phase of gait [74] or during loading, while facilitatory responses are evoked during gait [75].

4) At the end of stance the leg load diminishes and the suppression of the flexor center is relieved, thereby starting the swing phase, $[4,43,67,91,98,99]$. This is a process of disinhibition related to the removal of suppression of the SWG by the STG. The unloading is brought about by the extension of the hip but in addition some proprioceptive signal from the hip facilitates the onset of swing [100]. Indeed, later work showed that stretch of hip flexors facilitates the onset of swing [101] and the Ia afferents are the most likely contributors as detailed in [4]. These hip flexor afferents affect not only the levels of the motoneurons and pattern generators but also the rhythm generator level (figure 1b). Again, this is based on cat studies, showing in this case that entrainment of the rhythm is possible with manipulation of hip afferents $[102,103]$. For humans, the best evidence is from neonates as they lack a fully developed descending input from the brain. Indeed, in infants, hip extension induces locomotor 
movements [81]. In addition, in humans with spinal cord injury it was shown that stimulation of thigh afferents improved locomotor output [104].

Summarizing, proprioceptors (Ib and Ia afferents) play a key role in shaping the locomotor output in mammals, including humans. Work on transgenic mice has confirmed this picture [105]. In this latter study on Egr3 mutants (Early growth response protein 3, related to the regulation of biological rhythm) it was found that Group Ia/II from muscle spindles have a dominant effect on shaping the activity of flexor muscles, while Ib and group Ia/II are important for the patterning of extensor bursts (for an extensive review of this work see [106]). In the new flexor-dominated architecture of the CPG the extensor-center has limited capacity to produce intrinsic rhythmic activity. Rather the rhythmic extensor bursts originate from afferent feedback related to loading and from release of inhibition from the flexor center. This new notion fits well with the observation that the flexor burst is rather constant and that variations in speeds are achieved by changing the duration of the extensor phase $[45,91]$. In the light of these new insights it can be stated that locomotion is basically a periodic interruption of standing, whereby there is mainly a need for the periodic activation of flexors while extensors rely mostly on a mixture of load feedback from sensors and supraspinal facilitation.

Finally, a word of caution is needed. While for humans there is ample indirect evidence for a contribution of spinal CPGs [107], one should not overlook important differences. Bipedal gait in humans is slow to develop as it requires an upright posture, based on input from descending pathways $[6,108]$. Hence the contribution of descending input (Figure $2 b$ ) is likely to be especially important for human gait. Finally, as human gait is usually alternating, one needs to take into account the interlimb inputs as well (Fig 2b; see [106,109] for review). 


\section{Asymmetrical CPG model}

So far, most biped gait models based on CPG assume a symmetrical one with equal flexor and extensor parts. However, this situation is no longer valid. Recent physiological findings favor instead asymmetric models [43,110]. Based on animal data, Pearson and Duysens introduced a swing generator model [57]. In essence, this swing generator (or flexor burst generator) is tightly coupled to the rhythm generating system of the CPG (see figure $1 \mathrm{~b}$ ). The extensor generating neurons are activated by sensory feedback (especially from load receptors) and by a release of inhibition from the flexor burst generator $[4,55,67,70,72]$. In this new notion of CPG, the flexor circuits play a dominant role. The original support for this notion came from observations on the output of the CPG in various mammalian preparations such as cat or mouse. Flexor or extensor bursts can rarely occur without activation of their antagonist burst (deletions). Flexor burst deletions are accompanied by tonic extensor activity while instead flexor motor neuron bursting continues to occur during extensor burst deletions $[55,56]$. The evolutionary primacy of flexor pattern generation was confirmed with other techniques as well [17]. Not surprisingly, these new data have led to a change in the most advanced models of the CPG [45-47].

In the new flexor-dominated architecture of the CPG the extensor-center does not normally produce intrinsic rhythmic activity. Rather the rhythmic extensor bursts originate from a release of inhibition from the flexor center.

\section{Brainstem}

The new view of the spinal CPG comes in parallel with new insights about the role of the brainstem in the neural control of locomotion. Basically, the circuitry in the spinal cord allows for periodic flexion and extension of the legs and it has been argued that this circuitry could serve locomotion in various directions [111-113]. This was confirmed by work on 
decerebrate cats with epidural stimulation of the spinal cord. The animal's walking direction adapted to changes in treadmill direction, indicating that the sensory input changes were sufficient to adjust the locomotion mode [114]. Under natural conditions it is possible to hypothesize that this sensory bias is supplemented by descending commands from the brainstem [111]. In cats the evidence for this brainstem control was only obtained for one type of gait (forward gait), which depends on commands originating from the mesencephalic (or midbrain) locomotor region (MLR), as was shown originally by Shik et al., (1966) [115] and confirmed by others [114]. However, in recent years the role of the MLR has proven to be more complex than was suggested in earlier studies. The type of electrical stimulation, as used in the original studies [115] was rather crude as it recruited a number of cell populations. In recent years it was possible to make more selective activations or ablations and a new picture emerged (Figure $3 a)$.

In mammals, the MLR consists primarily of the dorsally located Cuneiform Nucleus (CNF) and the more ventrally situated pedunculopontine nucleus (PPN). Both areas are involved in slow speed locomotion, but CNF specializes in producing high speed gaits (as used in flight from a danger). The MLR contains 3 types of cells, namely glutamatergic (both in CNF and PPN, see figure 3a), GABAergic, and cholinergic cells [116].

Glutamatergic PPN neurons were seen to play a role in slow exploratory locomotor behavior [117] and these cells probably correspond best to the ones primarily stimulated electrically by Shik [115]. They receive input from basal ganglia nuclei, various midbrain sensory-motor nuclei and the motor cortex. In contrast, stimulation of the MLR GABAergic population caused cessation of locomotion and it was suggested that these cells are involved in freezing and fearrelated behavior [118]. These neurons receive input from limbic centers (amygdala, periaqueductal gray). 


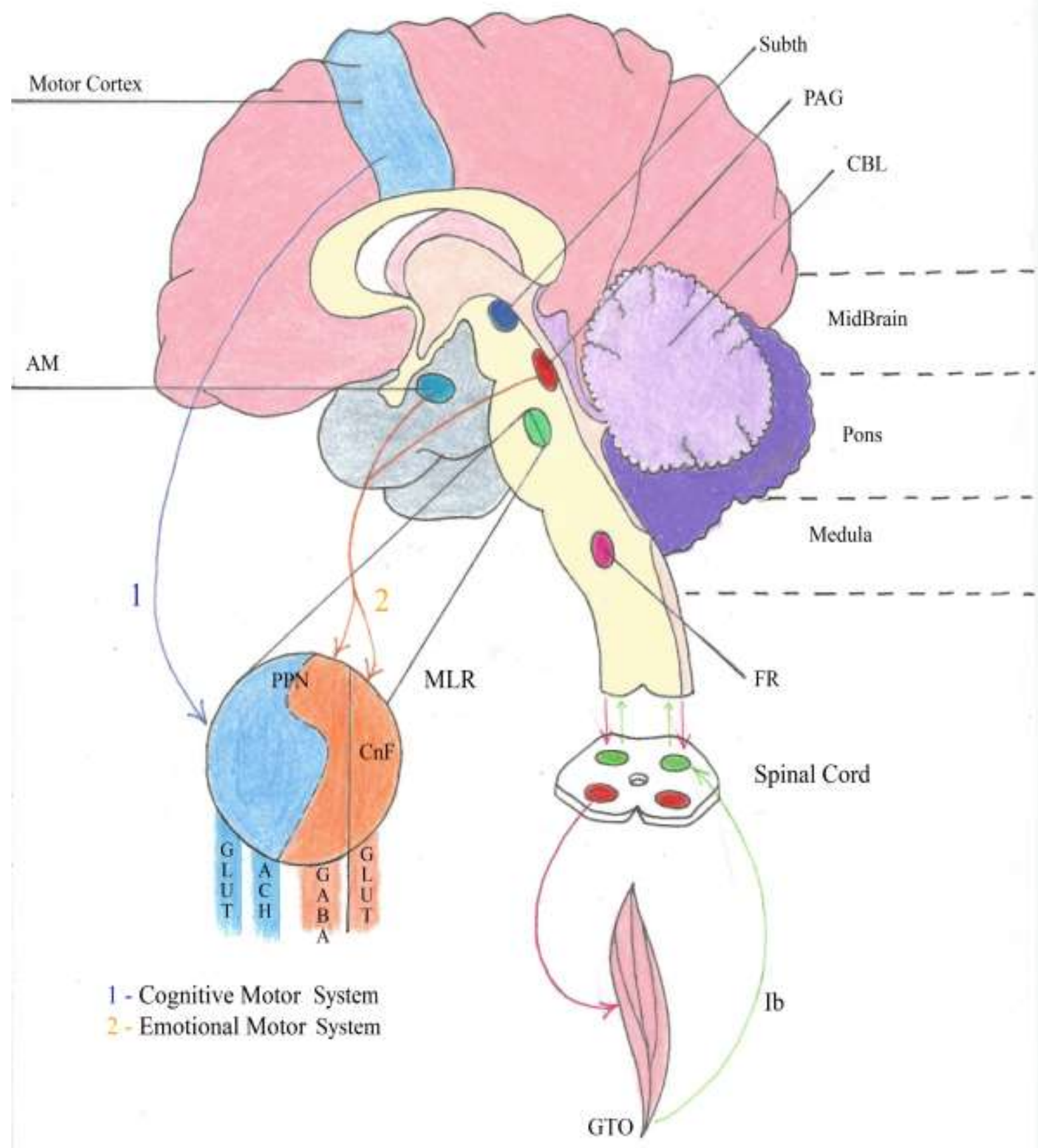

a) 


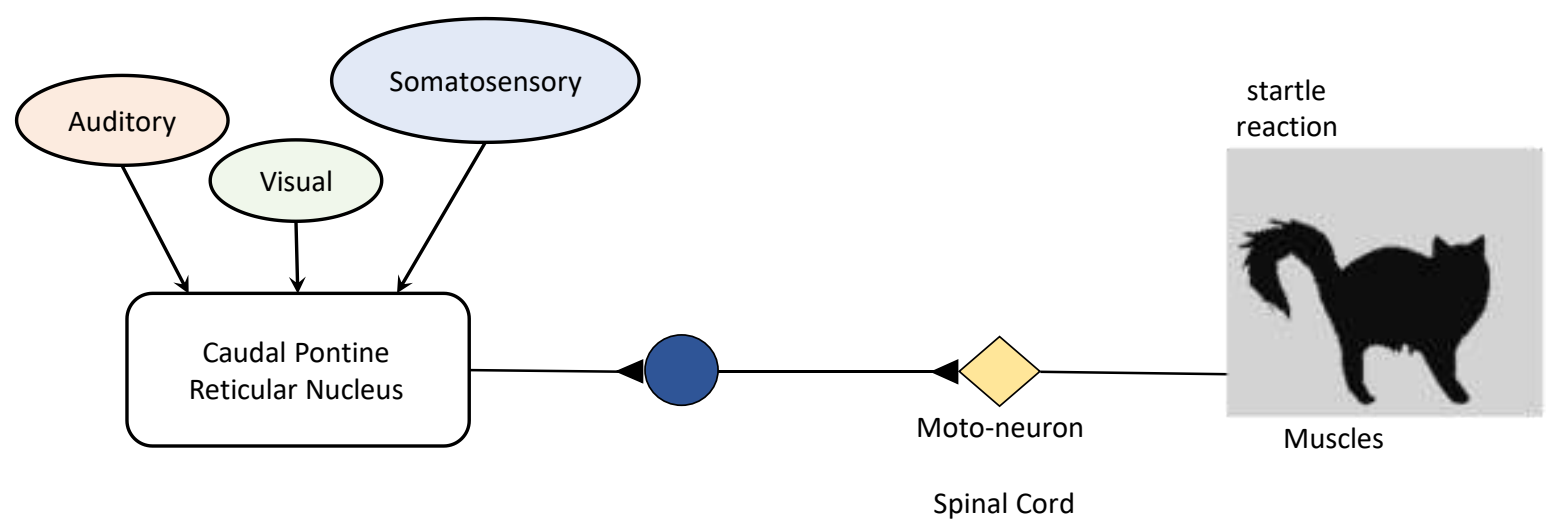

b)

Figure 3:

a) Schematic presentation of the various subpopulations and their function in MLR (mesencephalic locomotor region).

Under the control of the emotional motor system, the glutamatergic (GLUT) CnF (Cuneiform Nucleus) initiates and accelerates locomotion. These cells are linked to escape and flight reactions, as shown by the link with the Amygdala (AMG) and the periaqueductal gray (PAG). In the pedunculopontine nucleus (PPN) there are 3 groups of cells:

i. Glutamatergic PPN regulates slow-walking gaits;

ii. Cholinergic (Ach) PPN modulate speed and;

iii. Gabaergic (GABA) are responsible for gait termination.

The first 2 are part of the cognitive motor system (with input from the motor cortex) while the GABA part is responsible for freezing responses (emotional motor system). MLR receives input from the subthalamicus nucleus ("subth") and projects to RF (Medullary Reticular Formation) and from there the reticulospinal fibers descend to the CPGs (central pattern generators). Based on text from [119,120]. CBL: cerebellum. Load feedback is represented by $\mathrm{Ib}$ afferents.

b) Basic circuitry of the startle reflex. The nc gigantocellularis in the caudal and ventral part of the nucleus pontis caudalis receives input from various sensory sources and is involved in producing startle [121,122]. Adapted from [123].

Speed is another important variable to be controlled. In quadrupeds the slow speeds result in alternating gaits (walk and trot) while fast speeds result in gallop and bound (escape behavior). As mentioned above, the CNF is the part of MLR specialized in fast gait, in particular the Glutaminergic neurons (GlutNs) in that region. In contrast, slow gait depends on GlutNs in 
both of these regions (CNF and PPN). Other methods have confirmed and extended these findings. Using chemical stimulation, it was found that there is a topographical organization of the MLR.

For example, Sherman and colleagues found that there is an important difference between the ventral and the dorsal MLR [124]. Chemical stimulation of the dorsal MLR (including the CNF) induced walking, whereas stimulation of the ventral MLR caused standing and cessation of gait.

\section{Gait termination}

The termination of gait can be seen in three types of conditions, namely goal completion, fear and startle [125]. Termination of locomotor activity is a distributed function in the brainstem. At the midbrain the ventral MLR is concerned [124] (see figure 3a).

At the lower brainstem (pons and medulla) there is a contribution of the nucleus Gigantocellularis (Figure 3b). This structure is involved both in startle and gait termination. For the present review it is the startle stopping that is very relevant since it is a reaction to an unexpected perturbation, as can occur for robots as well. Such events lead to visual, auditory or somatosensory activations, all of which can influence the nc. Gigantocellularis as part of the medullar reticular formation (Figure 3b). It typically results in a brief interruption of ongoing gait [126]. In the section on perturbations several examples will be given of startle-like coactivations. As seen in Figure 3b, the three modalities project to the giant neurons in the lower pons (auditory over the cochlear nucleus, visual over the colliculus superior and somatosensory over the principal trigeminal nucleus for face input).The two levels (midbrain and pons) involved in stopping, are connected (Figure 3a). The reticular formation in the Rostral Medulla and Caudal Pons (with the nc Gigantocellularis) has been studied in detail in mice using new techniques, including optogenetic activation. Recently, a genetically identified group of V2a 
brainstem cells in mice was described as halting locomotion when activated optogenetically [127]. In other studies, it was shown that freely moving mice briefly stop walking when stimulating GABAergic cells in the pontine reticular formation $[118,128]$. These brainstem GABAergic cells project to the intralaminar thalamic nuclei (IL) and through this relay they can affect the cortex, more specifically, the frontal areas. These projections can ensure a complete transient interruption of ongoing behavior [128]. The lower brainstem, which receives input from the colliculus superior and the MLR [125], is also linked to higher centers in the brainstem. Hence, the new picture of the MLR is that it is heterogeneous and does not only control the onset of locomotion but also its termination by controlling the stop cells in the lower brainstem (Figure 3a).

This picture in mammals fits with recent observations made in invertebrates. For example, in lampreys Juvin et al. [129] found the so called "stop cells", a population of reticulospinal neurons that are activated at the end of a locomotor bout. These cells can be stimulated pharmacologically (using D-glutamate) and this stops ongoing swimming. In contrast, inactivation of the same cells impairs fast stopping. Hence across very different species it seems that "stop neurons" represent a glutamatergic descending pathway that favors locomotor arrest [130]. These reticulospinal cells are in turn controlled by the MLR, both in mice and in lamprey. In addition, as illustrated in Figure 3a, both the MLR Cuneiform Nucleus and the Gigantocellularis nucleus in the brainstem are connected to a "fear induced freezing circuit" including the amygdala and the midbrain periaqueductal gray (PAG) [131]. It is thought that such system could cause a brief freeze followed by a fright/flight reaction or described as "fast run following immobility" in [132].

How are these circuits affecting spinal cord neurons? Experiments with chemical stimulation throw light on this issue [124]. These authors studied the connections with the spinal cord. Their data indicate that flexor and extensor muscles were influenced by two separate 
neural circuits. One, which they termed an "extensor circuit" that regulates posture (mainly limb extensors) involving projections from the $\mathrm{SNc}$ (substantia nigra pars compacta) to the ventral MLR, a region also involved in termination of locomotion. The other, termed "flexor circuit", is essential for locomotion and is connected to the dorsal MLR. Again, this situation is consistent with the flexor centers being central to the generation of CPG activity.

The control of gait termination is important not only for humans but also for robots, especially when facing large perturbations. For example, it was found that stopping can be beneficial under certain conditions for robots. In one advanced robot, large instabilities were used to trigger a "stop walking reflex", involving a stop and a crouched posture, as is seen in humans in the startle reflex [133]. This "reflex" prevented the robot from falling.

\section{CPG and robotics}

Up to date, one of the most successful biomimetic models in the field of legged robots is the Central Pattern Generator (CPG). Such CPGs have originally been proposed for the cat [134] but they can be found in other species [135] and there is evidence for CPGs in humans as well [107]. Hence not surprisingly, many gait models include a CPG notion [136-148] and it was argued that the addition of a CPG helps to stabilize gait against perturbations [140]. Dzeladini et al. (2014) came to the same conclusion and they emphasized that CPGs offer the advantages of combining feedforward and feedback mechanisms, an essential feature in human motor control [149]. Furthermore, it is noteworthy that the idea of separate swing and stance generators (Fig 1b) was implemented in the latter model [148].

Nevertheless, there have been biped walking robots and models without CPGs. It is possible to implement a "feedback based locomotion" without the need of a CPG [150-152], although recently it was admitted by some of these authors that addition of a CPG could be beneficial to their models [153]. On the other hand, some CPG-based models claim that stable 
locomotion can be produced even with a large reduction in feedback activity [149]. A good reason to include a CPG in the models is that it allows the easy implementation of speed changes. In one of the earliest models the speed issue was solved by adding a tonic supraspinal drive $[136,154]$. From the cat work it is known that, depending of the intensity of the electrical stimulation applied to the mesencephalic locomotor region during locomotion, it is possible to produce gait at various speeds [115]. Hence this is an example how a spinal CPG can be controlled simply by one descending type of command, as initially proposed in the model of Taga $[136,154]$. Moreover, it is very interesting to see how the CPG can also be used to elicit the recovery responses by means of phase changes of the CPG, such as phase resetting (see further). The combination of reflex responses to modulate CPG phase in order to adapt to the environment or react to perturbations was previously described in different invertebrate and vertebrate preparations $[155,156]$.

There are two aspects in the CPG that are very important for its implementation that will be discussed in the text. First, the role of CPG as a local "internal model" of a rhythmic task, such as gait [85]. Second, in biped gait, there is a need to integrate information from different sources to maintain stability, hence the need for extra control systems. In contrast, in multilegged animals, CPG's have a more prominent role or even become the center of the locomotion control (such as in insects [6]). For an in depth review of the differences between human and quadrupedal gait and reflexes see [109].

There are many models of locomotion that use the CPG to generate gaits in robots and there are good reviews about this topic $[6,139,157]$. From a robotics perspective, a CPG is a non-linear oscillator or combination of oscillators that provide autonomously a rhythmic output pattern. The inputs to the CPG can turn the oscillations on and off, change its frequency and phase (including phase reset) or even change some of the parameters of the oscillators. These oscillators solve the problem of generating or synthesizing cyclic joint trajectories in real-time. 
When there are two or more legs with multiple degrees of freedom that have to be coordinated it is possible to couple the oscillators, so they have a certain phase lag and provide interlimb coordination. Moreover, when the robot has to move in unstructured environments with continuous variations of the speed, direction, and locomotor behavior [158,159] the motion of the legs follows stereotyped patterns with small variations from cycle to cycle related to the adaptation or entrainment with the environment, provided by the sensory feedback to the CPG [160].

From an engineering perspective, the CPG presents interesting properties, such as distributed control with fast control loops, capability to solve redundancies, disturbance rejection and modulation of locomotion with simple control signals [161]. These properties stem from the double nature of CPG. On the one hand, it can generate a pattern autonomously while on the other hand it can be modulated both by sensory inputs and by descending commands. One of the properties of the CPG models is to exhibit limit cycle behavior and produce stable rhythmic patterns. When there is a small perturbation that takes the system out of the nominal limit cycle but within the basin of attraction, the system returns to its rhythmic behavior thus providing robustness against perturbations. The artificial CPG, as its biological counterpart, has the advantages of robustness in the generation of patterns and can produce coordinated patterns of rhythmic activity while being modulated by control parameters [162].These properties are usually formulated as mathematical models and make CPGs very useful building blocks to implement locomotion controllers of biped walking machines $[163,164]$

The major problem in the use of CPG in robotics is the definition of the oscillator parameters, due to the non-linear behavior of the system and the frequent coupling between amplitude, frequency and phase. It is difficult to tune the CPG parameters of the robots. The 
techniques range from manual tuning to parameter optimization, using criteria such as a minimal energy consumption or maximal speed [165].

The implementation of CPGs in robots should consider the new level of understanding of the biological CPG structure and the interaction with sensory information and descending inputs from the central nervous system, in particular, the CPG asymmetry.

In conclusion, it is clear that there are plenty of examples of an application of a biological mechanism that was not completely understood, and the application was used differently in simulations and robotics.

\section{Sensory control of unperturbed gait in models and robots}

Not only is the notion of CPG now well-integrated in most model studies, there is also a lot of attention given to sensory input during gait, including descriptions in several detailed and recent reviews of the physiology $[6,8,109]$. Hence only a brief account is given here with emphasis on new data. A first distinction needs to be made between local and global control, a distinction that has only rarely been implemented in models, for an exception see [151]. This distinction is important, but implementation is often difficult since some afferents provide both local and global feedback [166]. The contribution of various receptors (tactile receptors, muscle spindles and Golgi tendon organs) to load feedback in stance has been extensively described by different research groups $[4,8]$.

The work in previous reviews was mainly focused on data from the cat. While humans and cats (and other mammals) share similar neural structures for locomotion control, there are a few relevant differences. Probably, the most important difference is related to the control requirements imposed by bipedal gait stability that is very different from quadrupedal gait stability. The increased complexity of bipedal gait stability is associated to a larger involvement of higher nervous system centers in the control of gait. Therefore, the focus of the present 
review is on humans along with an overview of the progress made in implementing the insights from physiology. Since the work of Frigon and Rossignol in 2006 [8], there have been many examples of models that incorporated some of these feedback features in simulations $[167,168]$ or in real robots $[140,152]$.

\section{Stance}

Role of Ia, II afferents. Position, speed feedback.

A first candidate for proprioceptive feedback during stance is provided by the Ia or group 11 afferents from the spindles in extensor muscles that provide, respectively, information about the rate of muscle length change and muscle length. When extensor muscles are stretched because of the weight-bearing at early stance, this local reflex prevents further lengthening by contracting the agonist muscle. The evidence that this is an important element in normal human walking is limited. Some groups estimated that, in the cat, a large percentage of the EMG of ankle extensors during stance is derived from this type of spindle feedback, up to $30-60 \%$ of EMG in [169] but others believe that it is relatively unimportant in humans [170]. The latter group showed that unloading the ankle led to a major reduction in EMG amplitude, even after blocking the transmission in Ia afferents that carry information about the rate of change in length $[83,171]$. This suggested that these Ia afferents did not contribute importantly to soleus activity during the stance phase of unperturbed gait [171]. In contrast, most experiments suggested a more important role for $\mathrm{Ib}$ afferents, that provide information about the muscle force from the Golgi tendon organs [83,172]. In the paper by Grey et al (2004) it was argued that length feedback is less important than load feedback for soleus muscle activity during human walking [83]. 
Role of Golgi tendon organs. Force feedback.

As explained above, the basic idea from the cat work is that during the stance phase the load feedback from ankle extensor muscles activates the extensor part of the CPG and suppresses the flexor burst generator [72]. At the end of stance the leg load diminishes and the suppression of the flexor center is relieved, thereby starting the swing phase $[4,43,67,91,98,99]$. It was suggested to use these insights in gait models $[1,6,9]$ and it is gratifying to see that there are now many models using some type of force feedback both in quadruped $[9,173-175]$ and in biped models [167,176-179] as well as in exoskeletons [180]. These models range from those that used only the inhibitory pathway [177], to others that used both inhibitory and excitatory connections [178] and some that use primarily positive force feedback [179]. In some recent models the feedback system worked so well that, initially, a CPG was not deemed necessary [179]. However, pure reflex models have difficulty explaining the changes occurring at different speeds. Indeed, it was shown that including a CPG in such cases improved the models dramatically as explained above [149]. Interestingly, the added CPG contained a feedforward component, in agreement with the model proposed by Kuo [85]. In this CPG model the feedforward component predicts the sensory feedback. This approach has a physiological basis and it reconciles two opposing views in motor control theories. On the one hand, the equilibrium point theories can easily integrate a $\mathrm{CPG}$ as a neural structure modulating the muscle activity [181]. On the other hand, the CPG, as an autonomous oscillator predicts the expected leg behavior acting as a local internal model of the task.

Some of the models mentioned above include an inhibition of the flexor center of the CPG by load feedback, e.g. for quadrupedal gait see the model of Fukuoka [175]. Finally, these concepts, borrowed from biology, are entering the realm of biped robots and some of them have load sensors to mimic Golgi tendon organs [140]. In this "neurorobotic" model the positive force feedback was one of the key features. This is in sharp contrast to the early attempts at 
modeling, where feedback from stretch reflexes dominated [182]. Even in this case, it was realized that some type of extensor reinforcing feedback was needed as well and it was provided by input from the vestibulospinal reflexes [182].

\section{Stance to swing transition}

At end stance there is not only unloading of extensors but also stretch of hip flexors. There have been quite a few examples of models and real robots using hip position or stretch as feedback signals $[138,152]$. Other models use primarily velocity of hip angle as feedback. For example, Verdaasdonk and colleagues simulated a semi- passive dynamic walker coupled to two CPGs controlling two hip actuators, one for each leg, and showed that delayed feedback of the angle and angular velocity of the legs helped in reaching a resonant state of CPG activation with optimal energy characteristics [176].

In summary, in robots and models the reflex-based gait control should take into account two important sources of afferent input: load receptors and hip afferents. The most important control involves signals about the loading and unloading of the limb. During the gait stance phase, the load signals increase extensor activity and suppress the onset of the swing phase $[4,72]$. At the end of stance, the limb loading decreases and swing can be initiated. A second signal relates to hip angle [102]. At the end of stance the hip extension is thought to assist the initiation of the swing phase using signals from the stretch of hip flexor muscles [101]. When both hip afference and unloading signals were compared in models, either of cats $[9,173]$ or of humans [183], only the unloading rule allowed the models to produce more robust walking.

\section{Swing phase}

An important notion for gait models is that the swing phase is not a uniform phase but consists of sub-phases $[184,185]$. Lifting the leg to provide foot clearance is but one aspect. Foot placement during touchdown is a crucial event. If the foot is placed too far forwards, then 
braking is too strong while if the foot is placed too far backwards, there is no adequate propulsion in the next phase. Hence it may be too simplistic to consider swing or stance as uniform entities. In models, it seems convenient to have separate systems or synergies for the various phases [186].

Such subdivision has a physiological basis. For example, in the respiratory system it is known that the generator for inspiration consists of separate groups of neurons for the initiation and maintenance of inspiration [187]. In particular, a distinction is made between earlyinspiratory neurons and late-E, late-expiratory neurons. This construction helps explaining some well-known reflexes as the Hering-Breuer reflex in which stretch receptors in the lung, thus sensitive to lung expansion, terminate the inspiration at the right moment, and not at another part of the cycle, when a different part of the generator is active.

During gait, the transition from flexor to extensor activity can be precipitated by foot contact but normally, the transition can be seen well before foot contact in what is termed the first extension phase (E1), the last part of the swing phase. Moreover, this can occur without supraspinal input. In spinal cats the extensor muscle activity was activated well before foot (paw) landing and able to produce enough force to support the body [86]. The signals needed to switch are likely related to hip flexion but the precise origin for the control of the swing-tostance transitions is currently unknown [188]. Possible candidates are the hamstring proprioceptors acting as hip extensors since their spindle Ia afferents are activated during the flexion phase of swing by flexion at the hip in conjunction with extension at the knee [189].

There is empirical evidence showing that these hamstring proprioceptors contribute to some of the hamstring activity in this part of the swing phase. Hamstring spindles are lengthened at the end of swing (in the first extension phase) and the EMG in these muscles is largely due to stretch reflexes [190-192]. It is a functional feedback loop since faster speeds need a stronger activation in this period. Since faster speeds mean faster stretch the result is an 
increase in activation with speed. Moreover, hamstrings are hip extensors needed for stability in early stance co-contracting with hip flexors. This appears as spring-like behavior and, in fact, modeling hamstrings as biarticular springs proved to be very successful [193]. The latter model demonstrated that ground clearance, leg retraction, reaching the desired angle of attack and preventing knee hyper extension can largely be achieved using biarticular springs tuned semipassively. Some other models also use hamstrings length feedback in swing successfully [179].

In models, it is common to postulate an anterior extreme angle or anterior extreme position to control the transition. The idea of an Anterior Extreme Position from hip signals, introduced by Cruse [194] was used in derived models [140,151]. Others are inspired by insect models and use local feedback as a main driving force without the need for assistance by CPG input [151].

The final phase of the swing, just before footfall, is particularly important in humans because, due to the foot anatomy, an uncontrolled heelstrike would lead to a sudden drop of the foot flat on the ground, named "foot slap". Instead, in healthy people, a smooth foot landing is performed. The available evidence indicates that for this sensitive action the role of supraspinal centers is essential, in particular the corticospinal pathway [195]. In this phase, the reflexes from various sources show phase-dependency because they are actively suppressed as if to avoid perturbation of landing [196].

\section{Phase-dependent modulation in models and robots}

An important element of feedback during gait is that one needs to control that feedback, depending on the phase of the step cycle. For example, a stretch reflex in soleus during stance is meaningful but not during swing [197]. Frigon and Rossignol [8] described how various models came very close to the cat physiology, in particular, the models of Taga $[154,160]$ and of Paul [178]. However, they also pointed out that there was not much progress in implementing 
the feature of phase-dependent modulation of reflexes. In particular, the models without CPG suffered from this omission. From the physiology it is known that feedback pathways depend heavily on the phase of the step cycle not only in cats $[57,198]$ but also in humans $[87,88,94,197,199,200]$. This feature is very important as it guarantees that reflex actions are appropriate for the phase of the cycle (i.e. a flexor reflex is fine in early swing but not at end swing when the foot is ready to be placed). This type of modulation depends either on spinal CPGs or on supraspinal influences [195]. In a later section the topic of gait perturbation is considered and again, the importance of the phase dependency of reflexes will be demonstrated.

\section{Generation of robotic biped gait: stability criterion.}

The generation of biped gait in robots is a major engineering challenge. Current biped robots are still far from their biological counterparts. Up to date there are many bioinspired solutions but only a few can be considered fully biomimetic. The field is so active that there have appeared tools to develop neuromechanical models that can be used to design robot controllers in a biomimetic fashion [201]. There are several control agents that should be considered in the design of a biped robot controller (see figure 4). Note that the center of the motion planning consists of a layer of rhythm/pattern generation (CPG-like) and a layer of step planning (equivalent to the motor cortex planning activity for foot placement, see above). As we have seen in previous sections, the CPG was proven to be a useful tool to generate coordinated step patterns with a certain level of adaptability to environmental changes and easy control from higher levels. However, to synthesize gait, these patterns should be integrated within a more complete structure that supervises the goal of the task or the path planning (both topics which are out of the scope of this review). 


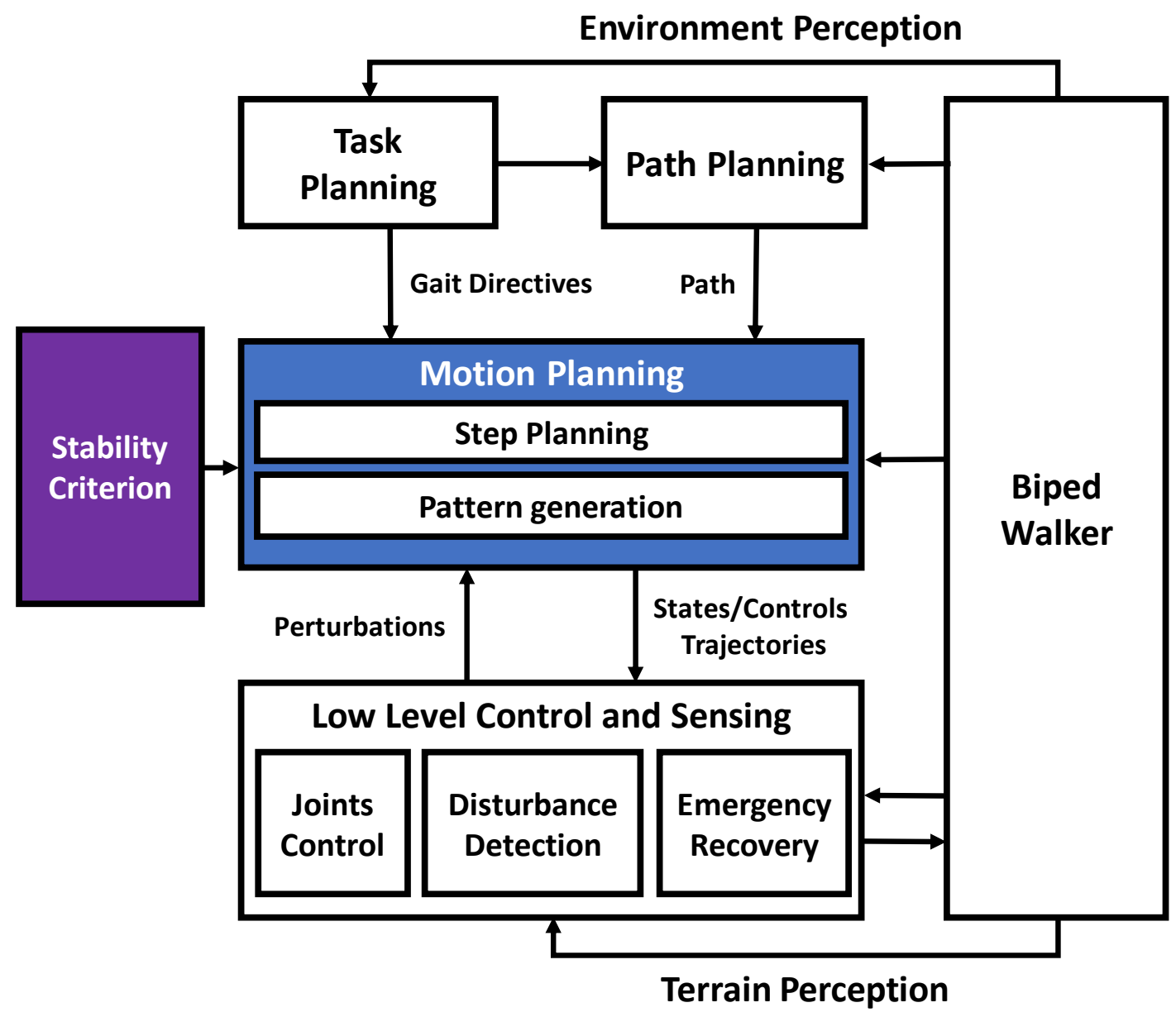

Figure 4. A general model of a controller for a Biped Walker, either a model or a physical robot. There are several levels in the controller. At the higher level the Task Planning defines the actions of the robot. From that information the Path Planning defines the robot motion considering the environment information. The intermediate Motion Planning module is responsible of the lowlevel motion of the robot, and defines the joint torques and trajectories taking into consideration the physical dynamics, constraints, torque limits, stability and environment characteristics. In this module there are two blocks, one to generate the step and the other to generate the rhythmic patterns (e.g. a CPG). The Step Planning needs to consider a Stability Criterion to guarantee that the biped will not fall. The Motion Planning provides the reference joint trajectories to the low level to effectively control the joints to follow the planned motion.

There were recent reviews comparing the different approaches used to generate stable locomotion in robots and in animals [6,202], hence a brief account and update is sufficient. 


\section{The problem of bipedal stability}

The analytical approaches are based on a model of the robot that allows the statement of the motion equations and the formulation of a walking algorithm based on these equations. This defines the biped trajectories mathematically and different techniques can be used to implement the control of the actuated joints. A stability criterion can be used to prescribe feet placement and reference trajectories for the joints in such a way that the robot can walk without falling. In robotics or models, most of the systems used analytical techniques in combination with another method, such as Neural Networks, in order to improve gait behavior [165].

There are three main groups of stability criteria in biped robots, (i) the zero-moment point (ZMP) that prescribe local stability at every point of the cycle [203,204], (ii) global stability methods that guarantee stability in the whole cycle [205] and (iii) the most recent approach using step capturability methods [206,207].

The first biped robot, WABOT 1, developed by Prof. Ichiro Kato in 1969, was able to walk a few steps based on a prescribed trajectory. In the same years, a theoretical development on the stability of dynamic systems was the Zero Moment Point, ZMP [203,208]. They proposed a sufficient stability condition for biped machines in which the point of application of the resultant forces under the feet lies within the convex hull defined by the feet [204]. This stability criterion has been widely used up to date to generate biped gait in robots since the eighties [209] until more recently [210-213] and it has been subject to several modifications in recent years [214]. The ZMP can be combined with other techniques to improve performance. However, due to the ZMP constraints, the robots walked with an unnatural slow pattern and with higher energy consumption than biological bipeds. In the ZMP, the trajectories of each joint should be closely tracked, thus requiring a precise trajectory control, this is very different from human gait, in which trajectories show some variability [184,215,216]. 
Lessons can be learned from the downhill walking robots consisting of passive biped machines that walk down a ramp with a stable limit cycle inspired another line of research [217]. The walking patterns are similar to those of a human both in appearance and in energy consumption [218]. Inspired by the passive limit cycle walkers, it was possible to develop Orbital Stability of periodic motions for biped robots. It must be noted that these robots do not need to be completely passive and the joint trajectories can show some variability (like in humans) provided that they remain within a certain basin of attraction around a Limit Cycle.

Feet placement plays an important role in human stable walking and recently there have been proposed several stability criteria based on the definition of adequate alternate feet placement, such as the "extrapolated Center of Mass" [219] or the step capturability [220,221]. The location of the feet also determines the position of the ZMP [222]. While in the classical ZMP approach it should be under the base of support so the biped could be stopped at any moment of the cycle without falling, in the step capturability, this point where the biped can stop is "somewehere ahead", much like in human gait.

Step placement is critical for successful bipedal gait in humans, either under normal circumstances, as proposed already in the eighties of the last century [223], or during the recovery from a trip [224]. The step placement techniques are based on calculating the points where the feet should be placed to avoid falling in both the sagittal and frontal planes. Usually, they are based on a simplified model of the body dynamics, such as an inverted pendulum. In the "extrapolated" CoM the stability criterion relies on an estimation of the predicted trajectory of the CoM and to maintain a certain relation with the Center of Pressure $(\mathrm{CoP})$, thus defining the feet placement at each step [219]. This stability criterion, based on the human gait, was based on the trajectory of the body center of mass $(\mathrm{COM})$ with respect to the base of support (BoS), using a simple model of the body as an inverted pendulum [219,225]. 
The most recent stability criterion is the N-step capturability [206,207,221]. In the capture point, the criterion is defined mathematically as the point on the ground where the foot should be placed in such a way that the robot can be brought to stop asymptotically [220,221].

Although the step capturability is not bioinspired, the concept seems biologically plausible. It is based on stepping in a certain region on the ground where the robot can be brought to a stable configuration. This idea paved the way to define another group of criteria for biped gait stability by means of appropriate foot placement [220]. These techniques are based on the definition of a set of points on the ground where the foot can be placed in order to perform a stable step. This idea, that is similar to the biological stepping strategy to regain stability, evolved towards the N-Step Capturability concept [221]. This criterion, that computes the steps a biped needs to stop from a given gait condition, is similar to the way as humans behave when they try to maintain or regain stability during gait [224,225]. Later, Englsberger used the "Divergent Component of Motion" [226] that can be extended to walking on an uneven terrain, as it allows the placement of the capture point anywhere in space, not restricted to the ground [227]. The step placement criteria have also been used to generate stable gaits in robotic exoskeletons [228]. Moreover, these criteria can be successfully applied to generate gait that can reject or recover from perturbations. For instance, the biped Atlas [229] or the DRCHUBO+ [230].

In the development of a stability criterion for biped robots, it is useful to understand human gait in order to mimic several desired features for biped robots such as robustness, versatility and energy efficiency [205,218]. It could be hypothesized that humans learn to walk in such a way that each step is completed safely and, in addition to it, the step should reach a certain position that allows future stable steps. The initial conditions for the next step allows for subsequent stable steps. This sequence of events is difficult to observe in normal walking conditions but it is apparent under certain conditions, such as after the recovery reaction from 
a trip [224]. Moreover, Matthis and Fajen [231] proposed that human beings achieve this behavior with the use of visual information to initialize the mechanical state of the body before the beginning of each step. Although it is not possible to determine which stability criterion is followed by humans, in this case, robot models and simulations can be useful to propose criteria that are biologically plausible and that can be used to help new concepts in the neurophysiology of gait control [232]. Therefore, a more detailed analysis of the stability criteria for the generation of gait will be provided in the Gait Perturbations section.

\section{Gait perturbations}

Maintaining gait when perturbations are present constitute a major challenge for both robots and humans. The term perturbation is used here in the broad sense for all conditions that require a deviation from normal gait to maintain stability. The perturbation origins (Figure 5) can vary from uneven or slippery terrain, to obstacles or pushes to the body. Irregular terrain poses many challenges as landing occurs on surfaces that are either higher or lower than expected.

It is essential to distinguish between expected perturbations (i.e. obstacles viewed long before a crossing is needed) and unexpected ones (suddenly appearing obstacles). In the former case the robot or human can anticipate the perturbation and adapt the gait accordingly (feedforward control). In the case of unexpected perturbations, the control is basically of the feedback (reactive) type, usually consisting of a series of actions described as "strategies". These recovery strategies, aimed at restoring the gait pattern or at avoiding the fall, share some features, and there are stereotyped responses to similar perturbations occurring in the same gait phase for different subjects. However, this must be taken with care because the responses can be adapted either to specific circumstances or to prior subject experience (learning effect). 
Here another distinction needs to be made, namely between small and large perturbations. Small perturbations do not need a complete phase-resetting of the gait cycle, while a large perturbation would require a change of the step strategy. In other words, small perturbations do not need to reset the CPG and the gait pattern can continue with small adjustments. In contrast, large perturbations require a phase resetting. In all these cases the reactions of the human subjects consist of a series of muscle activations, many of which are below the threshold latency of voluntary reactions [233,234]. These reactions are a first line of defense and therefore worthwhile considering when designing equivalent reactions in models or robots.

\section{Models and robots}

As many models deal only with unperturbed gait there are relatively few authors that have tackled the problem of gait perturbations in simulations. Initially only small perturbations were considered $[136,161]$ but gradually more attention has been given to larger events such as slips, obstacle avoidance and tripping and pushes [152,160].

Various types of perturbations (Figure 5) will be discussed one by one. First an overview of the physiology is given and then the implementation in models and robots will be discussed. One great challenge for models and robots is to overcome obstacles while walking. Here a distinction has to be made between successful avoidance and failed avoidance (tripping). Failure depends on whether obstacles can be anticipated or whether they appear suddenly such that anticipation is minimal because of time pressure. 


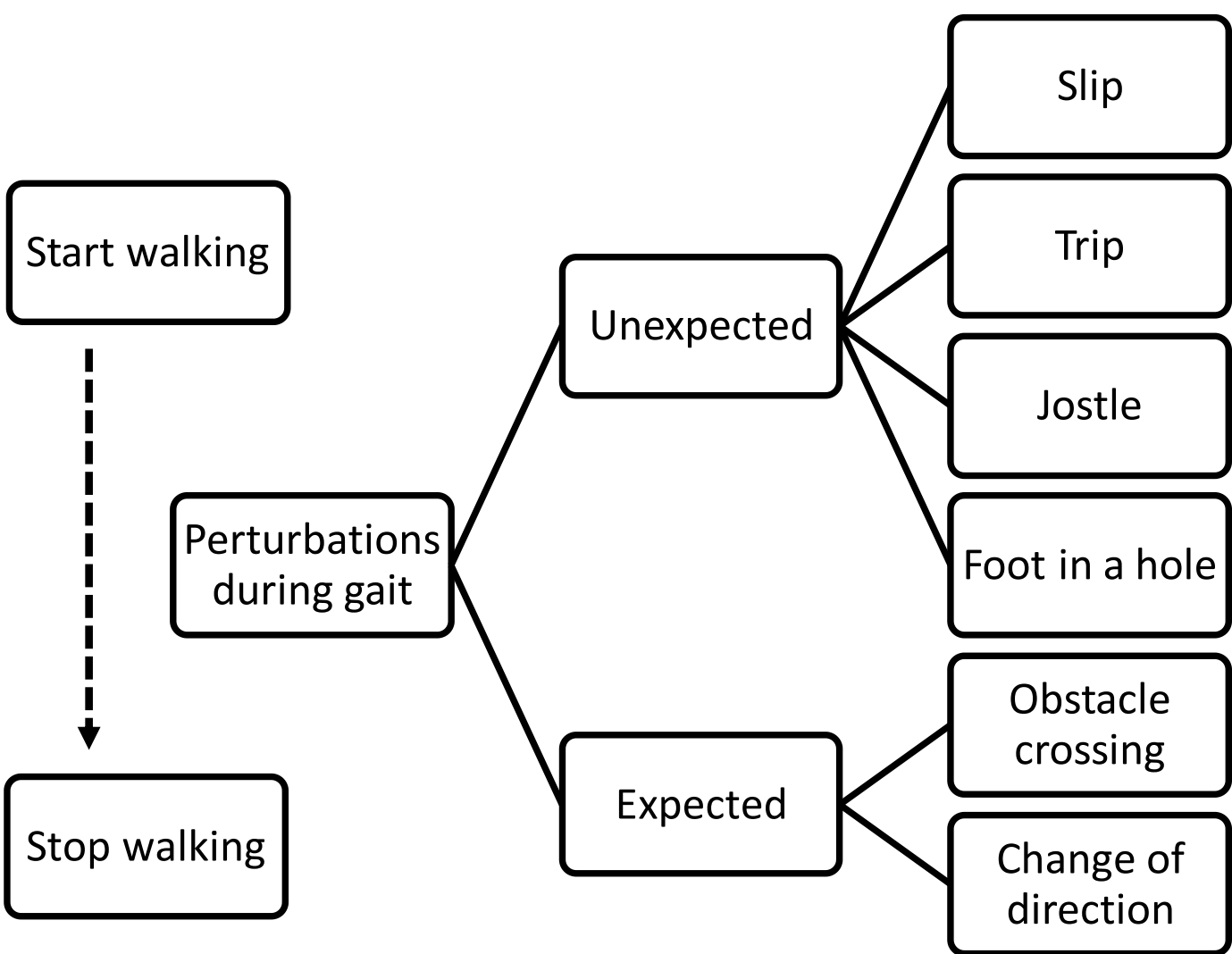

Figure 5. Taxonomy of gait and perturbations during gait.

The study of gait perturbations also allowed to propose theories about the control of the step, which have been used to control biped robot stability. As mentioned above, initially the emphasis was on the zero moment point (ZMP) as a representation of the point of application of the ground reaction forces that should not go beyond the borders of the support to maintain contact with the ground [208]. Although this notion has been very helpful it could not account well for dynamic stability challenges (such as a push given during walking). This issue of dynamic stability is of primary importance both for robots and humans faced with sudden gait perturbations. It is therefore perhaps not surprising that key notions about balance control appeared almost simultaneously in the field of robotics and biomechanics. In both cases the starting point was the linear inverted pendulum model. In biomechanics, Hof and colleagues introduced the now widely used notion of 'extrapolated center of mass position', named XcoM 
[225]. It was proposed that for stable gait the position of (the vertical projection of) the CoM plus its velocity times a factor (square root $1 / \mathrm{g}$ ) should be within the BoS, 1 being leg length and $\mathrm{g}$ the acceleration of gravity.

Slightly later, but independently, a similar notion was developed in the robotics field. Pratt and Tedrake introduced the 'Capture Point' (CP) as the point on the floor where the robot should place its foot to stop despite perturbations, in other words "the region on the ground where a humanoid must step to in order to come to a complete stop" [220]. Hence the approach taken by Pratt [220] and later by Englsberger [235], was mainly aimed at bipedal push recovery, while Hof used his concept more broadly [225], an example followed by many biomechanics authors since then. Later, Hof proposed that for stable walking after a disturbance, the foot should be placed such that the center of pressure CoP lies at a certain distance behind and outward of the extrapolated center of mass at the time of foot contact. More recently the work on Capture Point dynamics was extended by the notion of model predictive control (MPC), a control method based on the modeling and anticipation of future events to take relevant control actions on time [222]. MPC was applied to push recovery control in gait as well [236]. These developments in forward control show that robotics is indeed approaching neural control principles at a fast rate.

One of the most important neural principles was proposed in 1950 by von Holst and Mittelstaedt, the efference copy [237,238]. This is a copy of a given motor command, allowing to compare predicted sensory feedback with the real sensory feedback. This is particularly useful to suppress self-generated afferent input (see figure 2a). Such neural forward models have been applied to biped robots such a RunBot [239,240]. 


\section{Anticipating obstacle avoidance}

Visual input is especially important when walking over uneven terrain and avoiding obstacles [241]. When sufficient time is available the subject can prepare the avoidance. Adult humans direct their gaze to an obstacle some 2 steps in advance [242-244]. Nevertheless, this is not an absolute figure because, when the terrain is more complex, gaze fixations become closer [244]. During obstacle crossing, the role of vision depends on the leg used [245]. In the lead limb visual information can be available for on-line control while this is not the case for the trail limb. For the lead limb the timing of the visual input is critical for accurate foot placement. Visual information about the ground surface or obstacle has to be sampled after midstance of the preceding single-support phase. It is not needed after foot liftoff [231].

Peripheral vision is also important in obstacle avoidance, especially vision from the lower hemifield [246]. This is especially true when time-pressure is involved [247], such as in fast avoiding reactions to an approaching obstacle [248]. A special role is attributed to peripheral vison in the context of evaluation of the position of the lead leg with respect to the obstacle. The term "visual exproprioception" is used to indicate vision of the lower limb in relation to the environment. It has been argued that visual exproprioceptive information is used on-line to fine tune the lower limb trajectory during obstacle avoidance [249]. Later studies specified that such information is more important with respect to final foot-placement than to update toe-clearance during crossing [250].

Similar to unperturbed walking, the weight given to a given form of sensory input during obstacle avoidance depends on availability. When vision is restricted or abolished one can still avoid obstacles provided haptic information is added, for example such as provided by a cane [251]. When tactile information about obstacle position can be gathered beforehand, the crossing of the obstacles can also be performed successfully [252]. Otherwise, adjustments to 
the obstacle avoidance can be found. Subjects with impaired vision use higher toe-clearance and increased distance between foot and obstacle at foot placement [249,250]. It can also be noted that these adjustments are different between blind and blindfolded people, indicating a long-term adaptation to visual deprivation [252].

The physiology and neural control of avoidance of expected obstacles was described by McVea and Pearson (2009) for cats [188] while for humans the avoidance of unexpected obstacles was recently reviewed by Potocanac and Duysens [253]. When obstacles are expected one can prepare and plan the appropriate action. In brief, the vision of an expected nearing obstacle is analyzed in the parietal cortex, which also is informed about the current state of the body and has a memory about obstacle locations. The command to overcome the obstacle are sent to the motor cortex. In contrast, the avoidance of unexpected obstacles may rely more on subcortical pathways (see section 3.2). In either case, expected or unexpected, the role of vision is crucial.

The same applies during running over uneven ground with obstacles. Human runners use their vision to plan their encounter with a heightened or lowered surface at least 1 step ahead [254,255]. On uneven but visible terrain, runners compensate actively almost half of ground level changes (40 to 50\%) one step ahead [256]. This anticipation, in conjunction with a number of passive mechanisms [257] yields a largely unchanged vertical CoM height despite the uneven terrain. Hence, controlling the CoM vertical variations appears to be a general control principle in running. The term "disturbance rejection" is used to describe the effort to limit the effect of perturbations on the body center of mass (CoM) trajectory [258]. This theme is important in the context of robotics. For example, in the robot Meltran II the length of the legs are adjusted such that the $\mathrm{COM}$ is at a constant height above the walking surface (for this and similar examples see the book of Westervelt [259]). An important factor to be considered here is the positioning 
of the "hip" with respect to the CoM: a posterior positioning increases potential extension torques but makes the robot more unstable [260].

\section{Models and robots:}

Model studies on obstacle avoidance are faced with the need to have a tool to perceive the obstacle and based on that information to calculate the required anticipatory actions.

For biped models one of the first attempts was made by the group of Winter [261]. They used the data from previous experimental gait perturbations with one subject as input for their model and for validation [261]. They studied unilateral obstacles of $10 \%$ of the subject's leg length (this was almost $9 \mathrm{~cm}$ for the subject used) and visual input. Pattern-generating algorithms produced an estimation of anticipated contacts with obstacles. This was used to establish weighting functions that created the joint angle trajectories and joint torques needed to overcome the obstacle. Slightly later, Taga proposed a model of the neuro-musculo-skeletal system for anticipatory adjustment of human locomotion during obstacle avoidance [160]. It was proposed to use a "discrete movement generator", which receives both the output of the CPG (named RG, rhythm generator, in their case) and visual information about the obstacle, thereby providing commands to alter the basic gait pattern. A crucial element was that time-tocontact to obstacles was used (based on the rate of divergence of optical flow) and secondly, that the phase of the gait cycle was taken into account. Increasingly in recent times, the models are based on synergies and obstacle avoidance is achieved by adding or modifying synergies [262] or by superimposing voluntary activations on the underlying walking pattern, such as the superposition of a new pulse command to a set of invariant pulses (see figure 6, [168]).

In robots, one particularly successful attempt in obstacle avoidance was the HONDA robot, which can take a step in response to obstacles of $150 \mathrm{~mm}$ height and $150 \mathrm{~mm}$ length [263]. This robot has video cameras for robot or obstacle position estimation. 
It should be noted that solutions are not necessarily bioinspired and the ZMP-based robots cross obstacles with a strategy which is very different from the human with vision. In particular, keeping the hip backwards while crossing the obstacle is the preferred control strategy of bipedal robots using the Zero Moment Point stability criterion [232]. Interestingly, such behavior is also seen in blindfolded subject, when avoiding obstacles [252].

\section{Unexpected perturbations}

In this section the conditions are described where humans or robots encounter perturbations during locomotion that could not be anticipated.

\section{Foot-in-hole}

When subjects or robots step unexpectedly in a hole, the instant of foot contact differs from the expected one. Such conditions have been imitated in the laboratory [264,265]. Van der Linden and colleagues described a fast functionally relevant muscle synergy (a stop walking synergy), caused by the mismatch between the expected and actual sensory feedback at the foot contact instant [264]. The results were thought to be consistent with an internal model, possibly as part of a cerebellar pathway. Another group made a similar study but they used unexpected loss of ground support by removing a wooden board mounted on the walkway [266]. They basically found similar responses as in the study mentioned above but in addition they investigated the recovery afterwards. The gait rhythm was reset, and the subsequent take-off was delayed, allowing the human to continue walking. In this study and in a subsequent one [265] they also found that prior knowledge about a "potential hole" made the subjects perform the task differently, implying the addition of a learning effect. To avoid this problem of anticipation, some experiments [266] had only one perturbation trial for each subject in a study on unexpected stepping down ("foot-in-hole experiment"). Another approach was recently taken by a group that used a treadmill with a belt having an adjustable stiffness [267]. They 
found that subjects could continue to step but that the leg contralateral to the perturbation made a shortened swing phase.

Foot-in-hole experiments were also performed on running humans. When the hole was visible the subjects anticipated by pre-activation of ankle extensor muscles prior to foot contact $[254,255]$. When the hole was camouflaged the subjects landed with increased impact (as was also observed in a study on walking, [268]). In addition, contact time was shortened and there was an increased angle of attack and leg length with drop height (in analogy with running birds, [257,269]). The activation of the muscles tested (Gastrocnemius medialis, vastus medialis, tibialis anterior) increased with the height of the camouflaged drop after landing. Some activation was also seen just prior to foot contact (as in the experiments on walking of Van der Linden, [264]). However, this should be interpreted with some caution since the subjects repeated the camouflaged trials and therefore could anticipate a possible drop.

\section{Foot-in-hole for robots}

The equivalent of humans walking with a foot-in-hole has not been tested systematically in biped robots but some attempts were made to add sensors to the feet to enable walking on uneven terrain [270]. In contrast, several groups have tackled the issue of stair descend, which is particularly challenging as it requires lateral tilting [271-273]. Probably most spectacular is the stair descend of robots such as Asimo [274]. Interestingly, the latter authors made use of a phase-dependent sensory feedback controller.

\section{Suddenly appearing obstacles}

When an obstacle suddenly appears, the risk of losing balance and to fall increases. Not surprisingly this topic has caught the attention of many people involved in research on falls in the elderly, but it is also increasingly important for the field of robotics and gait models as it adds an extra component of time pressure. Sudden obstacles can be of various kinds, from plates 
or ropes suddenly appearing on a walkway to wooden blocks or ropes used to perturb the swing phase while walking on a treadmill, thereby halting the leg during the swing phase of the gait cycle [275]. The studies on humans have been recently reviewed by Potocanac and Duysens [253] and it was proposed that attention should be given to fast subcortical pathways since they allow for very fast corrections with latencies that are well below those needed for voluntary corrections (as used for anticipated obstacles, see above). Such subcortical pathways may have developed for crucial situations, requiring fast detection along with fast responses [276-278]. Hence, visual detection has two phases, the fast detection of stimuli that are crucial for survival (i.e. detection of a snake) and the slow detection of details less critical ("not a harmful snake"). The motor reaction for the fast detection are also of 2 kinds. The first reaction is unspecific (startle, freeze) while the second one is specific (fight, flight, avoidance) [126,233]. As mentioned above, the brainstem plays an important role here (in particular the nucleus reticularis gigantocellularis). In tripping responses for example, the first reactions are not specific with respect to the avoidance strategy, but later responses are highly specific [233].

Sometimes the sudden obstacles can be avoided successfully but in other cases not, in which case tripping occurs. In all cases the reactions to finally overcome the obstacle fall into 2 categories [279-282]. Either humans immediately make a large step over the obstructing obstacle (elevating strategy) or they first put the foot down and then later lift if over the obstacle (lowering strategy). These reactions are phase-dependent in that elevating is typically seen for perturbations in early swing while lowering is a feature most commonly seen in late stance [253]. In addition, in some cases the recovery requires several steps. The stumbles induced by pulls of a rope during swing while walking on a treadmill allowed the analysis of the recovery across several steps [282]. Later, they analyzed the segmental energy changes in the recovery from a stumble [283]. In the elevating strategy, the energy loss induced by the stumble was restored during the perturbed step and reached normal levels during the recovery step. The 
largest energy changes occurred in the lowering strategies because more strides and larger energy changes were needed to recover, suggesting a trade-off between stability and energy efficiency [283]. Elderly people may opt for stability as it was found that older adults (65-75 years) always adopted a lowering strategy when perturbed with a tripping stimulus in late or mid-swing (60-80\%), while younger adults also adopted elevating strategies [284]. Simulations by these authors showed that, when perturbed later in swing, subjects needed a larger recovery step and higher recovery limb force for successful recovery. The insufficient recovery limb strength made it difficult for older adults to produce a sufficiently large recovery step for a successful elevating strategy recovery. Similarly, using a rope to induce tripping, it was possible to find the switching point between the two strategies. It can vary between subjects and is also dependent on the duration of the pull, in the case of tripping with a rope. This duration was varied systematically and it was found that pulls of longer duration produced tripping most closely resembling trips on real objects [285]. As perturbation length increased, the transition from elevating to lowering strategies occurred at earlier perturbation onsets.

Planning future steps is an important part of perturbed gait. For instance, when stepping over obstacles, the distance from toe to obstacle is kept at about $60 \%$ of the total step length. This guarantees a safe landing and adequate conditions to perform the following step [286]. In addition, it was shown that the recovery from a trip is performed in several steps [282]. Each recovery step should be completed stably while reducing the effect of the perturbation and guaranteeing that the subsequent step can also be stable [224]. In this way the recovery can be performed efficiently in terms of the required torque, impulse, power and torque/time [287]. Moreover, it was also proposed that the step length is tuned in such a way that it creates a sufficient moment arm to apply torques on the trunk without need to accelerate the Center of Mass (COM) of the body [224]. If the step is too short, as it occurs when tripping, it is not possible to apply a torque to control the trunk flexion using only the vertical ground reaction 
forces. In such a case, it is needed to apply horizontal forces that accelerate forwardly the COM and require a subsequent faster step [224,288]. During normal gait, the step length guarantees that it is possible to control the trunk without the need for horizontal forces.

\section{Models of bipedal gait}

These experimental notions are now gradually being adopted in various models. For instance, in 2011, Forner-Cordero et al. [289] used a musculo-skeletal biped model to simulate tripping by the application of forces to stop the swinging foot . Recovery reactions were simulated by adding muscle excitations to selected muscles of the legs, and the recovery reaction only improved with the activation of the iliopsoas, a muscle that cannot be measured with surface electromyography and it is not measured in the trip recovery experiments [289]. Similar muscle-driven simulations were performed by Klemetti and colleagues [290], using subject-specific muscle-driven simulations of young healthy subjects walking at one of four speeds. Tripping was simulated by applying a constant backward force to the swing-foot of the model. They concluded that fast walking is not necessarily more unstable than slow walking, with respect to responses to a constant perturbation. However, one should be careful in extrapolating these results, because the conclusions may be valid for the speed range used but in a broader context it should be recalled that high speed is a risk factor and that response time is important. For example, in their tripping study, Pavol et al., (2001) studied kinematic and kinetic variables describing the recovery attempts and they compared subjects who fell with those who recovered [291]. It was concluded that fast walking may be the greatest cause of falling following a trip in healthy older adults. An anterior body mass carriage (stooped posture), accompanied by back and knee extensor weakness, may also lead to falls following a trip. In this particular case, deficient stepping responses did not contribute to the falls [291]. Nevertheless, in many other cases, response latency remains important. This was assessed with 
a simpler model, consisting of an inverted pendulum, which showed that response time was more important than the absolute walking speed to have a successful recovery response [292].

Hence, a crucial element for these studies is the latency of the responses. For unexpected obstacles these responses are faster than voluntary ones, but they also depend on the level of expectation. Since perturbations are usually repeated over time in the laboratory it is unavoidable that some degree of expectation affects the results. For example, in the study by Forner-Cordero and colleagues [234], using constraints of the swing phase by a rope, the responses occurred faster as perturbations were repeated. Similar results were reported by Shinya et al, [293], using sudden acceleration or deceleration of a split-belt treadmill. They found that onset latency of the gastrocnemius response was about $20 \mathrm{~ms}$ shorter ( $72 \mathrm{~ms}$ vs. 58 ms) when subjects knew the timing of the upcoming perturbation. These results indicate that the excitability of supra-spinal pathways can be increased by knowledge of the timing of the forthcoming disturbance.

Such finding are explained by considering the various possible cortical and subcortical pathways involved in descending commands [253]. Some models and robots started to implement such pathways. In the model of Jo [294] the reactions to tripping are seen as long latency responses evoking a descending pulse that excites different sets of muscles depending on the phase of the step cycle [295]. For perturbations in the early swing phase, the pulse activates the flexors in the swing leg (elevating strategy) while perturbations in late swing activate muscles that produce a lowering strategy. The Jo and Massaquoi study further detailed this model by including a loop over the cerebellum [167]. In their SBBW model (Sagittal control of Bipedal Balance and Walking) the phase of the cycle is sensed, and the model goes as far as implementing presynaptic inhibition in the (simulated) spinal cord to assist phasedependent modulation. In the biped model of Tomita and Yano [296], the phase is detected by sending the input from external afferents, as to position, load and force of each muscle to the 
CPG. In addition, there is interaction between afferent input and basal ganglia, allowing flexible control of muscle tone and locomotor output much along the line of the findings from animal research by Mori [297]. The great benefit of such models is that they allow adaptability to environmental changes, thereby creating robustness to unpredictable changes of environment. Unexpected load changes on the body can be detected and the relevant information is sent to the basal ganglia-brainstem system, which in turn can adjust the elasticity of the legs.

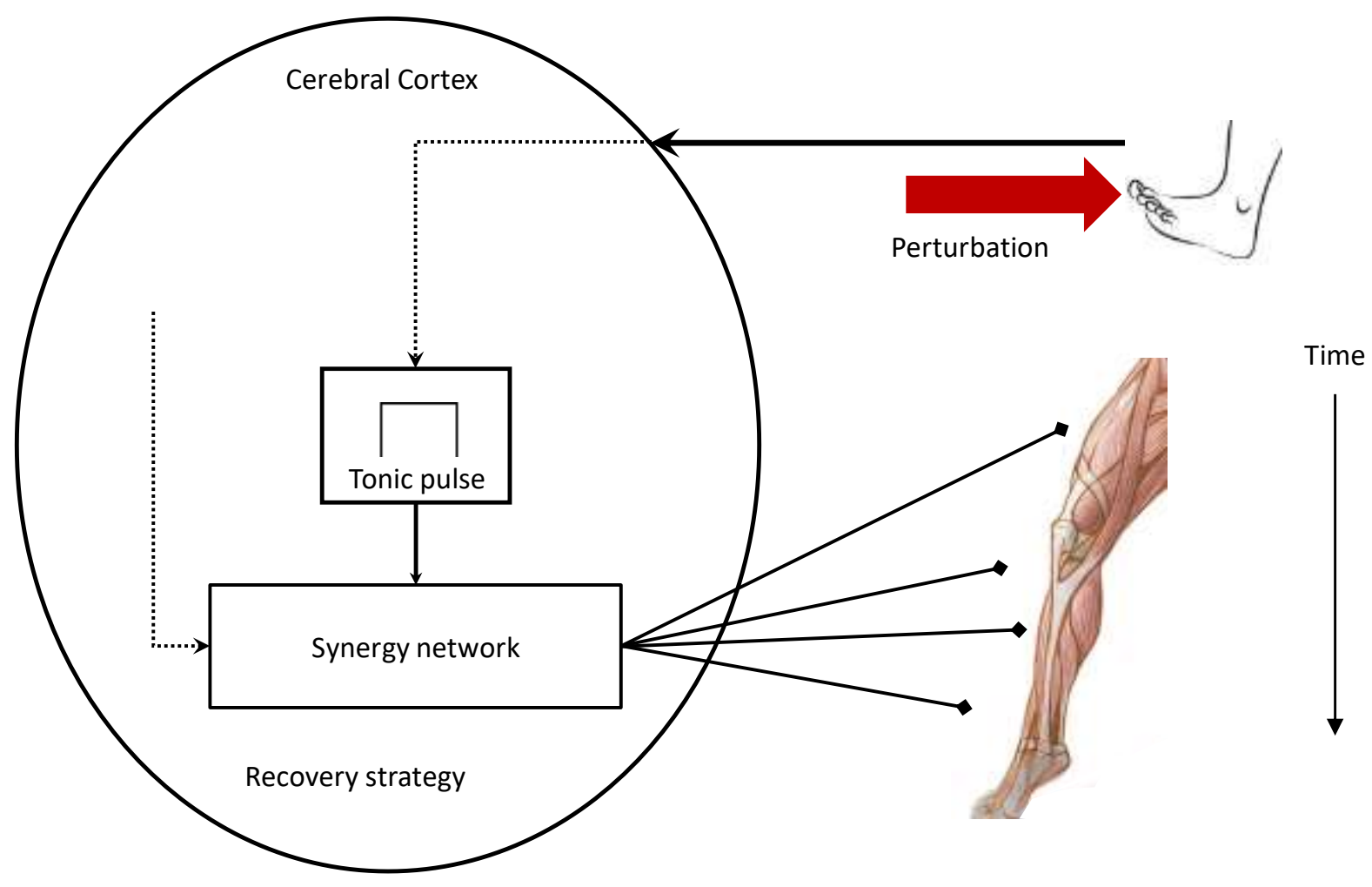

Figure 6. Scheme explaining reactions to perturbations. Adapted from the model of Jo and Massaquoi (2007), which uses a pulse generator, which could be involved in a long-latency reflex running over the cortex and generated by a perturbation at the foot [167]. The results is a tonic pulse, produced at brainstem level and projected to the spinal cord and to synergy-generating circuits. 
In the "neurorobotic" model of Klein and Lewis [140], the biomechanics, CPG and sensory feedback were used to control a robot, which fully models walking in a biologically inspired manner. The CPG and phase modulated reflexes were simulated using a spiking neural network. Klein and Lewis [140] also showed the advantage of using a CPG because this assisted in stabilizing the gait against perturbations and this was measured relative to a purely reflexive system. Advanced cat models also account for phase-dependent modulation $[173,186]$. In this case the CPG is constructed as a neural phase generator (NPG), a system of a series of states (along the line of the Forner-Cordero idea of a sequence of states, see above), controlled by a single control neuron representing descending input. The NPG is informed about the phase of the step cycle and thereby can control the feedback pathways so that only actions are allowed that are appropriate for the particular stage of the step cycle. This is especially important in case of gait perturbations since it simplifies control. A single descending input is sent to the NPG and is translated into different responses, depending on the state of the spinal circuitry, following a suggestion by Drew [295] based on microstimulation of brainstem in walking cats.

\section{Synergies}

The fact that the correcting responses in obstacle avoidance were termed "strategies" may not have been a coincidence when it was common to think in terms of control over a subset of muscles. Indeed, in the field of motor control there has long been the idea that motor control is simplified by using a given set of muscles, now the so-called "synergies" (see previous sections). Gait is not only seen as a sequence of states defined by kinetics and kinematics [184] or a sequence of tasks, [185] but also as a sequence of synergies. In recent years this idea has been implemented extensively by the group of Ivanenko and colleagues [32,262,298-300], for review see [30]. They showed that walking in the newborn relies on just two synergies [300] but, in healthy adults, the EMG activity during locomotion can be represented by five separate periods of $\mathrm{MN}$ activation, related to major events in the step cycle (heel strike, weight 
acceptance, loading/propulsion, trunk-stabilization activity during the double support phase, liftoff). As acknowledged by these authors, each one of these phases has a different relationship with respect to afferent input during the step cycle. As each phase evolves, new afferent input is provided that allows for automatic switching between the phases of the gait cycle.

The synergy approach is currently entering the field of gait simulations as well. Meyer and colleagues [301] used a patient-specific synergy-controlled neuromusculoskeletal simulation framework to predict walking of a post-stroke patient. They used 5 synergies per leg to predict successfully the subject's walking kinematics and kinetics. This idea about synergies was also adopted by some groups to explain reactions to various types of perturbations such as tripping [167]. These authors used an earlier model as basis [302], to consider responses to pulling a swing leg. They reproduced the two main responses to tripping (elevating and lowering) by generating them as synergies resulting from a tonic pulse from the cortex reaching the CPG at different times of the step cycle (see figure 6). A detailed review of synergies in gait models is available [303].

Slips

A very common type of gait perturbation is provided by slips. In the laboratory, slips can be reliably induced using surfaces of low friction [304,305] but most commonly a form of artificial slipping is evoked either by walking onto a moving force plate [306,307] or by speed changes on a treadmill [308-312]. It should be mentioned that some of these simulations may be less "natural" than others. Troy and Grabiner [313] compared slipping on ice with surrogate slipping tasks and found some important differences (in parameters that differed between successful and unsuccessful trials) which made them conclude that "slips induced using artificial ice more accurately reflect the type of slips that occur in the community". Nevertheless, the use of these other techniques has been quite informative. 
For example, in the field of slip responses and synergies the approach of the group of Ting yielded important insights. Chvatal and Ting [306] simulated slips by translation perturbations of the support surface during walking. Locomotor muscle synergies could be shifted in time to account for responses to the slips. Hence, they mostly needed a flexible recruitment from a library of muscle synergies. In a follow-up study, Sawers et al. [307] used muscle synergy analysis to compare differences in muscle coordination between older adults who fell and those who recovered from a slip. They found that subjects who fell recruited fewer muscle synergies than those who recovered. The fallers had delayed knee flexor and extensor onset times in the slipping leg, as well as a different muscle synergy structure. One striking feature was that the fallers had greater bilateral muscle activation and more muscle coactivation, both characteristic of a startle "freeze" contribution.

The synergy approach has clearly a lot of potential [314] (see also the review by Ivanenko, Poppele, and Lacquaniti [30]), for example by offering an explanation for gait on slippery surfaces [315]. Muscle activity during human gait can be accounted for by four to five modules/synergies. When faced with slippery surfaces, the gait of subjects changed, and EMG modules were characterized by a widening of muscle activity patterns, presumably to increase stability.

In line with Aoi and colleagues $[303,316]$ it should be stressed that the neural basis for the locomotor synergies remains weak. One exception is the flexor synergy, as has been recognized since the early days of neurophysiology [43]. Furthermore, a major criticism on the theory of synergies is that muscle synergies might reflect task constraints rather than a neural control strategy [317]. It was concluded that task constraints and performance optimization are sufficient to explain synergistic muscle recruitment from a redundant set of muscles. For a recent discussion on the use of synergies in robotics, see Lacquaniti et al. [318], 
A final comment is in place about the use of the unperturbed leg and the arm movements in reactions to slips. In humans the reactions to slips are whole-body responses involving a bilateral upper and lower limb response [305,319]. In particular, the flexion of the slipped leg is combined with an extensor response in the contralateral leg (to provide stability). The arms are lifted, and this arm elevation helps in shifting the center of mass anteriorly to counteract the shift due to the slip. In addition, Tang and Woollacott [319] emphasized that these reactions are phase-dependent, as is common for all reactions to gait perturbations. These are important features to be taken into account for the construction of models and robots.

\section{Models and robots}

One commonly used approach in robotics in the context of slips is to introduce active disturbance rejection [321]. The latter used a "disturbance rejection controller" in conjunction with a general dynamic model of 3D biped robots. Following the disturbance rejection, the leg joints keep their desired trajectory and the robot foot does not slip.

Another approach is to model the responses themselves. For example, to get insight into the neural control of slip-responses a modelling approach (see Figure 7) was taken by $\mathrm{Yu}$ and Ikemoto [320,322]. They used muscle activity profiles acquired from human gait experiments for their model which was based on CPG-phase-modulation along with a rapid responding pathway. The simulated walker was able to maintain balance when confronted with slipperturbations. In a subsequent study [323] they evaluated the relative importance of the 2 key features namely muscular reflexive patterns and phase modulation. Their results indicated that the muscular reflex is effective for the immediate balance recovery, while CPG phase modulation is important for a fast recovery to a stable walking cycle. 

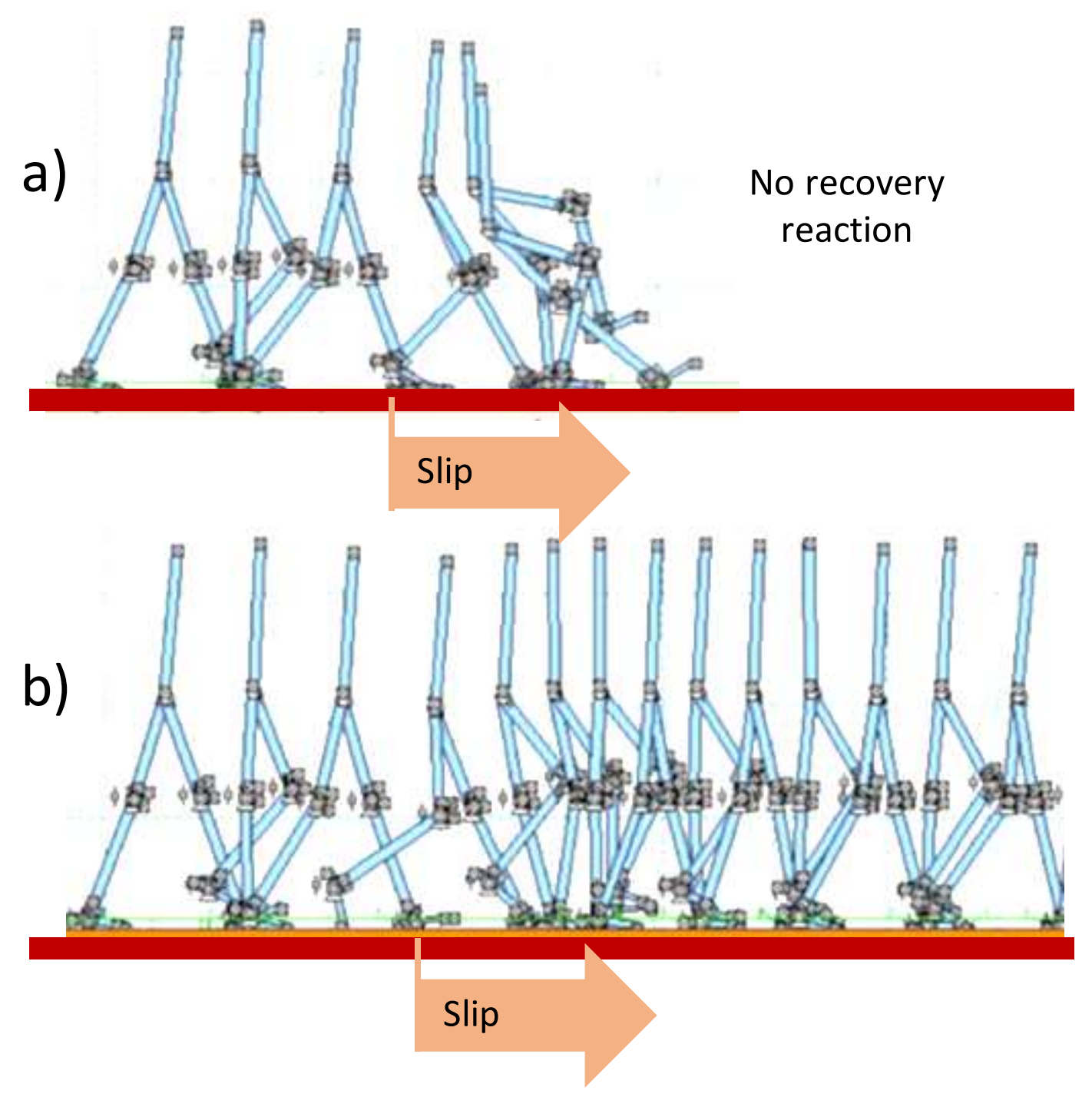

Figure 7. Slip recovery in a robot using the reflex response approach. Stick graphs comparing a model of a robot suffering a slip during walking and resulting in: a) a fall if no recovery reaction is implemented and b) walking without falling with a reflex-based recovery reaction with reflexes. Adapted from Yu and Ikemoto [320]

Some robots have also acquired slipping and tripping "reflexes". For example, Boone and Hodgkins [324] instrumented their biped robot with a set of responses. For slipping there were two types of responses, one-step strategies and two-step strategies, depending on whether the correction was applied in the perturbed step or in the following one. The tripping involved 
a box which the robot could step onto after sensing the contact. In this paper an extensive review was given of the state of the art, until the date of publication, of attempts at modeling robots that can deal with slips and trips.

A different suggestion for a reflex control method for biped robots to quickly recover their posture from a slip soon after a detection of the slip came from Park and Kwon [325]. They used simulations with a 12-DOF biped robot and a 6-DOF environment model and claimed that the proposed method was very effective in preventing a fall-down of biped robots on a slippery surface. Kajita and colleagues, based on a humanoid robot HRP-2, calculated the minimal friction of the slippery surface needed to walk successfully, resulting in a friction coefficient of 0.14 [326]. Most solutions involve open loop control, yet some attempts were made for online trajectory control as well and this was shown to effectively enhance the stability of locomotion [327].

\section{Recovery responses from gait perturbations in robots; a question of stability}

Usually, the problem of eliciting a recovery response during gait in Robotics is split in two. First, detect the perturbation, such as the slip, and then implement an adequate reaction, that could be a recovery or a preparation for the robot toa fall.

Back in 1997, Boone and Hodgins proposed the use of reflexes, seen as an unspecific fast response directly driven by sensory inputs, to react to perturbations such as tripping and slipping [324]. The idea of implementing reflexes in robots is not new [328,329] and it should be noted that the implementation of these robotic reflexes in physical robots was not based on a deep understanding and replication of the biological reflexes. In biological systems, reflexes have different response time scales (short and long loops) and integrate different types of information both at spinal (one or several segments) and supraspinal level. Furthermore, in 
robots there is usually no phase dependent modulation of reflexes, while this is an important feature in biological systems [195].

The issue of maintaining stability of robots when faced with perturbations has been approached very differently in past years. In robots, initially gait was based on ZMP trajectory control which did not provide stability in the light of perturbations. More recently however, there have been attempts to compensate for induced ZMP deviations, such as using a ZMP feedback control to control the angular momentum of the body [330] This was further refined by another group [237] to generate feasible joint trajectories that guarantee ZMP stability. An approach to reject disturbances is based on ZMP compensation that could be implemented by applying a compensating torque at the ankle joint; the calculation of this torque was based on signals from force sensors located at the four corners of each foot (as applied on a humanoid robot, MANUS-I) [331]. A more natural compensation acts at the level of the trunk or arms, as proposed by some robotics authors [332,333]. Modified arm motion is also a feature of the approach taken by Hill and Fahimi [321]. These authors argued that compensation for a disturbance requires 2 steps: the robot must react to (compensate for) the disturbance, and then return to the desired gait. Another example of this can be found in simulations where the trunk and the arms of the robot are used for stabilization against external perturbations [334].

Finally, models are also useful to predict fall conditions and, for example, compare the predicted threshold for backward balance loss following a slip with experimental data collected from a large sample of young adults [335]. They used a 7-link, moment-actuated human model to predict the threshold of the center of mass $(\mathrm{COM})$ velocity relative to the base of support (BOS) required to prevent backward balance loss during single stance recovery from a slip (evaluated at liftoff of the trailing foot in gait). There was good correspondence between the two [335]. 


\section{Cocontraction}

One of the striking differences between biology and gait models is that in the real word one frequently can observe instances when antagonists are coactivated. In gait models, such coactivation is avoided since it is energy inefficient. However, in humans there sometimes is a trade-off between energy and safety concerns. Therefore, it is essential to check the instances when coactivation is often observed. Again, the focus will be on perturbations.

It was argued that coactivation produces functional stability by decreasing oscillation and overshoot through the control of the total stiffness of opposing muscle forces [181]. This should lead to a minor freezing reaction which may have evolved because of a survival strategy [278]. However, whether this is always functional remains to be seen. It depends on how well this freezing is incorporated into the ongoing activity. In extreme forms (in clinical syndromes such as hyperekplexia) it is clearly dysfunctional [336] but in many other instances, in normal subjects, it is usually not disturbing and may carry the benefit of allowing extra processing time. Consistent instances of coactivation were first seen in standing subjects after platform rotations. Using a "toe up" or heel down" rotation, one might expect a stretch of ankle plantar flexors which should lead to a stretch reflex in these muscles [337,338]. The expected contraction was seen indeed but also a concomitant cocontraction of the antagonist muscles (tibialis anterior). These cocontractions remained basically unexplained.

During gait, a brief period of cocontraction/freeze may be functional as it minimally affects the ongoing gait process. This is seen during perturbed gait initiation [339] or when auditory startle responses were elicited during gait [126]. Other different kinds of perturbation can elicit similar brief coactivations of ankle flexors and extensors. For example, it is seen when stepping on an unexpectedly lower surface [264,266], avoiding a "forbidden zone" after tripping [253,340], walking with sideways pushes [341] or with simulated slips [307,312]. Interestingly, the startle-like coactivation was frequently seen in the subjects that lost balance, 
indicating that this might be a dysfunctional response. However, it can also be argued that in these cases there was some protective function related to other startle-like actions, such as the flexion of the head and the bent arm posture. In addition, the delay introduced by the brief freezing may be beneficial in assisting phase resetting, which contributes to generate robust bipedal walking [342]. Finally, it is worth noting that in many cases the slip trials actually consisted of the first, unexpected slip [307]. This is important as one will see in the next section where it is argued that cocontraction is prominent, in particular in unexpected perturbations (“first trial effects") but also in preparing for upcoming perturbations.

\section{First trial and learning effects}

Robots and gait simulation models share with real subjects the fact that they fall sometimes. As this is an undesired outcome it is useful to learn what is essential for prevention of falls. Some expectation of a possible upcoming perturbation is a crucial element. To illustrate this point, it is of interest to look at a study showing that the expectation of an upcoming large postural perturbation influences the recovery stepping response and outcome [343]. Following a perturbation (treadmill belt acceleration in $150 \mathrm{~ms}$ ), $47 \%$ of subjects fell when they were not expecting the perturbation, whereas $12 \%$ fell when they were aware that the perturbation would occur "sometime in the next minute". Hence these data suggest that in future work it may be of interest to include a "learning aspect" in robots and simulations with the idea that "one perturbation predicts another one. This learning may include the possibility of reducing the latency of descending commands as in [294].

Latency is of primary importance for successful recovery. It seems that the subjects, when surprised by the perturbation, had slower reaction times than the subjects that were expecting it [343]. Given the slower tripping reactions of elderly as compared to young adults [281] along with a slower rise in force, it is not surprising to see that elderly are much more likely to lose balance after a trip [288,344]. 
It may be argued that robots or models do not need to learn since the perturbation when they appear are, in essence, unexpected. However, in real life it is not uncommon to encounter multiple obstacles and perturbations during gait (given an uneven surface for example), hence repetitions and learning are not unnatural.

On the other hand, it is also useful to realize the effects of surprise. It is a common feature of many gait perturbation studies that they make a distinction between the first (unexpected) trial and subsequent ones. As pointed out by Troy and Grabiner [345], when perturbations are repeated this provides wide opportunity for the subject to anticipate and plan for the perturbation. They went as far as having only a single trip for each subject (causing a "lowering strategy" after a rope induced stumble). The same protocol was applied in a study on unexpected stepping down in foot-in-hole experiments [266] . Nevertheless, many researchers reported that basic response patterns persist after repetitions, despite some differences with the first response that could also be attributed to an exaggerated response. In one study, such first response (first trial) was compared to startle responses in the same subjects and many similarities were noted [336]. In particular, early masseter activity persisted, which indicates a startle-like component of the response. Moreover, focusing on the first trial response was found to be no better than analysis of pooled responses to a series of perturbations [346]. In fact, as was pointed out in the latter study, valuable data is missed by concentrating only on the first trial since balance reactions can be improved by repeated exposure. For example, it has been reported that the subjects modified the responses to laterally directed disturbances during gait within 12 trials [347]. Even basic response characteristics such as latency may change as perturbations are repeated [234,293]. It is important to consider these differences even though they are usually small. Pijnappels [348] investigated whether changes in lower limb muscle activity occurred during gait in anticipation of a possible trip. Significant but small differences in averaged muscle activity were found, more specifically, slightly increased co-contraction 
mainly in hamstrings, quadriceps and tibialis anterior muscles. Another study, using a walk over surfaces of different friction, found that awareness of a potential slip altered the approach of the slip-limb [349]. Prior slip experience affected the anticipatory muscle activation and the way the foot interacted with the floor (consistent with a more cautious gait). In robots it may also be useful to switch to a more cautious gait when perturbations are encountered repetitively.

Even when taking these precautions, falls will not always be avoided, and this raises the question whether robots should learn to fall safely. In elderly people, it was shown that it is possible to learn to fall more safely using fall techniques borrowed from judo [350]. Such techniques reduce substantially the impact from a fall [351]. Robots with arms should be able to learn such fall techniques as well, because falls can cause serious damage to biped robots. This field is relatively new but there were already some interesting studies [352-355].

In general, inspiration is taken from $u k e m i$, the technique of falling safely. The strategy involves braking the fall by spreading impact and by landing on the ground at one of the shockabsorbing parts of the robot. For example, Ma et al. [355] investigated four strategies based on human protective falling motion (knee flexion, torso flexion forward, torso translation backward and knee stretched strategies). These perform negative work, thereby reducing the energy and the impact velocity of the robot). In the study by Renner and Behnke [133], socalled reflexes were added to assist safe landing. For small instabilities the robot slows down but continues walking. For stronger instabilities the robot stops ("stop walk reflex") and its mass is lowered by flexion in the legs. Walking continues as soon as the instability disappears. The latter stop reflex shows striking similarities with minor freeze and startle responses during human gait, as described above. 


\section{Robot and human learning}

Although robots may be taught to fall safely, it is better if they could learn to avoid falling while walking. Indeed, when faced with walking in an unpredictable environment the best solution is to include learning to adjust the responses. In humans this can take the form of acquiring new synergies [300] during development. In the adult motor control system, the main advance is to include a possibility of comparing expected and real outcome of stepping. It is thought that the cerebellum has a dominant role in this and one way of testing this involved split-belt treadmills. The use of such system was promoted by the group of Dietz [356,357] but its use in adaptation studies came much later [358-364]. With a split-belt it is possible to have asymmetric steps with belts at different velocities. In the original studies on split-belt walking of humans, it was found that humans could easily adjust to walking with each leg at a different velocity [356], but how this was achieved was not known. The group around Bastian discovered that intralimb parameters (such as limb excursion; i.e., the distance the foot travels along the belt during the stance phase) adapted rapidly. In contrast, the interlimb parameters (such as step length and double support time) adapted more slowly and exhibited marked aftereffects when the belts were returned to equal speeds after a period of walking with different speeds [358].

These adjustments were thought to rely on the intact cerebellum, since cerebellar patients showed these changes much less clearly, at least when the patients were affected severely $[360,361,363,365]$. Some of the fast learning was attributed to the spinal cord since spinal cats were shown to be able to adjust to split-belt walking [198].

Given this background one can imagine that the idea arose to incorporate cerebellum features in walking models and bipeds. In one of the most advanced cases, Fujiki [366] used a two-layered control model for walking composed of spinal and cerebellar circuits implemented in computer simulations and in a bipedal robot. In the spinal model a central pattern generator was used with feedback from foot contact sensors. In the cerebellar model some form of 66 
learning was introduced based on differences between predicted and actual foot contact. They found that both early and late adaptations of split-belt walking could be reproduced along with after effects.

\section{Robots and models with arm motion}

While it will take some time before robots will learn to use their arms successfully to brake a fall (see above), a start has been made already in introducing arm swing during robot gait. It is well-known that humans use arm swing to neutralize the angular momentum created by the leg movements [367]. Passive walking biped robots have been instrumented with swinging arms and it was claimed that this improved stability [205,368]. In active robots some groups started to add arm swing to biped runners as well [210]. It remains for future work to construct robots that use their arms successfully to compensate for unexpected perturbations during gait.

\section{Conclusions}

The biological inspiration in the control of gait has been very useful to improve the design of walking robots. However, this is a bidirectional way, as the design of bipedal gait of robots has been useful to understand the control of human gait.

One example of success is the generation of rhythmic behaviors in robots (such as gait) based on CPG. A refinement in the implementation of this technique included the concept of asymmetry and the recovery from perturbations with techniques such as phase resetting or phase advance.

On the other hand, robots can also be used to test possible stability criteria used by humans, as these cannot be directly measured. In this respect, the gaits resulting from the 
application of ZMP criterion are unnatural, while other methods, such as the step capturability, are more biologically plausible.

More interestingly, and less explored up to date, the robotic implementation of techniques to avoid obstacles, to recover from perturbations or even to fall as safely as possible, as found in humans could lead to a leap forward in biped robot gait. In this respect, the implementation of reflex-based recovery responses or learning how to fall safely are examples of biomimetic techniques that improve the robotics fields.

There are several ways/avenues for future development of biped robots, for instance, see Chapter 14 on Bio-Inspired Robot Locomotion from Buschmann and Trimmer in [369]. Some improvements can stem from the mechanical design based on bioinspiration, for instance, to increase compliance in the actuators or soft robots. Compliant actuators include elastic elements in series with the actuator. Moreover, the design of distributed modular actuators could be very useful to improve robotic efficiency and make it more similar to human gait.

The biomimetism in control design is based in the implementation of a hierarchy combining the central and peripheral mechanisms, thus combining lower-levels autonomy with higher-levels control based on global body information such as in the internal models of motor control. This distributed hierarchy is useful when dealing with multiple degrees of freedom with actuators and sensors. While humans have a large number of degrees of freedom, actuators and sensors, current robotic technology do not. However, new robotic skin allows the inclusion of many sensors. It is necessary to have mechanisms to filter and use this information.

Moreover, biomimetism is usually regarded as a desired feature. However, human beings have evolved to maintain a motor system that allows a high degree of flexibility and robustness: It is necessary to determine the real need for this flexibility (from playing violin to 
cut down a tree; from dancing on top of the tiptoes to running while kicking the ball) in a biped robot.

Finally, we must consider for future biped robots as well as understanding of human gait, the more general perspective of artificial intelligence (AI) to create robots that look like or even "are" intelligent. AI can be used to generate gait and even to detect small gait alterations that reflect possible pathological conditions or even the state of a certain person.

Overall, it is believed that the future biomimetic approach will combine an innate CPG with robotic learning abilities, anticipatory control and reflexes to achieve a stable biped gait.

\section{Acknowledgements}

JD was supported by a visiting professorship from the National Research Council of Brazil (CNPq, grant number: 400819/2013-9). AFC acknowledges a CNPq research grant (grant number: 311055/2016-8). We acknowledge Clara Ploretti Cappatto for the help with the figures. 


\section{References}

[1] Duysens J, Van de Crommert H W A A, Smits-Engelsman B C M and Van der Helm F C T 2002 A walking robot called human: Lessons to be learned from neural control of locomotion J. Biomech. 35 447-53

[2] Forner-Cordero A, Pons J L L, Wisse M, Ceres R and Calderón L 2008 Basis for Bioinspiration and Biomimetism in Wearable Robots Wearable Robots: Biomechatronic Exoskeletons ed J L Pons (Chichester, UK: John Wiley \& Sons, Ltd) pp 17-45

[3] Fayemi P E, Wanieck K, Zollfrank C, Maranzana N and Aoussat A 2017 Biomimetics: Process, tools and practice Bioinspiration and Biomimetics 12011002

[4] Duysens J, Clarac F and Cruse H 2000 Load-regulating mechanisms in gait and posture: comparative aspects. Physiol. Rev. 80 83-133

[5] Büschges A and Gruhn M 2007 Mechanosensory Feedback in Walking: From Joint Control to Locomotor Patterns Adv. In Insect Phys. 34 193-230

[6] Buschmann T, Ewald A, Twickel A Von and Büschges A 2015 Controlling legs for locomotion - Insights from robotics and neurobiology Bioinspiration and Biomimetics 10041001

[7] Jayaram K and Full R J 2016 Cockroaches traverse crevices, crawl rapidly in confined spaces, and inspire a soft, legged robot Proc. Natl. Acad. Sci. 113 E950-7

[8] Frigon A and Rossignol S 2006 Experiments and models of sensorimotor interactions during locomotion Biol. Cybern. 95 607-27

[9] Pearson K, Ekeberg Ö and Büschges A 2006 Assessing sensory function in locomotor systems using neuro-mechanical simulations Trends Neurosci. 29 625-31

[10] Grillner S and Wallén P 2002 Cellular bases of a vertebrate locomotor system - Steering, intersegmental and segmental co-ordination and sensory control Brain Res. Rev. 4092 106

[11] Grillner S 2018 Evolution: Vertebrate Limb Control over 420 Million Years Curr. Biol. 28 R142-3

[12] Jung H, Baek M, D'Elia K P, Boisvert C, Currie P D, Tay B H, Venkatesh B, Brown S M, Heguy A, Schoppik D and Dasen J S 2018 The Ancient Origins of Neural Substrates for Land Walking Cell 172 667-682.e15

[13] Don E K, Currie P D and Cole N J 2013 The evolutionary history of the development of the pelvic fin/hindlimb J. Anat. 222 114-33

[14] Yokoyama H, Ogawa T, Shinya M, Kawashima N and Nakazawa K 2017 Speed dependency in $\alpha$-motoneuron activity and locomotor modules in human locomotion: indirect evidence for phylogenetically conserved spinal circuits Proc. R. Soc. B Biol. Sci. 28420170290

[15] Falgairolle M and Cazalets J R 2007 Metachronal coupling between spinal neuronal networks during locomotor activity in newborn rat J. Physiol. 580 87-102

[16] Saltiel P, d'Avella A, Tresch M C, Wyler K and Bizzi E 2017 Critical Points and Traveling Wave in Locomotion: Experimental Evidence and Some Theoretical Considerations Front. Neural Circuits 1198 
[17] Machado T A, Pnevmatikakis E, Paninski L, Jessell T M and Miri A 2015 Primacy of Flexor Locomotor Pattern Revealed by Ancestral Reversion of Motor Neuron Identity Cell 162 338-50

[18] Cazalets J-R, Borde M and Clarac F 1995 Localization and Organization of the Central Pattern Generator for Hindlimb Locomotion in Newborn Rat J. Neurosci. 15 4943-51

[19] Kjaerulff O and Kiehn O 1996 Distribution of networks generating and coordinating locomotor activity in the neonatal rat spinal cord in vitro: a lesion study. J. Neurosci. 16 5777-94

[20] Kiehn O and Kjaerulff O 1998 Distribution of central pattern generators for rhythmic motor outputs in the spinal cord of limbed vertebrates Ann. N. Y. Acad. Sci. 860 110-29

[21] Bonnot A, Whelan P J, Mentis G Z and O'Donovan M J 2002 Spatiotemporal pattern of motoneuron activation in the rostral lumbar and the sacral segments during locomotorlike activity in the neonatal mouse spinal cord. J. Neurosci. 22 RC203

[22] Bonnot A, Whelan P J, Mentis G Z and O'Donovan M J 2002 Locomotor-like activity generated by the neonatal mouse spinal cord Brain Res. Rev. 40 141-51

[23] Hagglund M, Dougherty K J, Borgius L, Itohara S, Iwasato T and Kiehn O 2013 Optogenetic dissection reveals multiple rhythmogenic modules underlying locomotion Proc. Natl. Acad. Sci. 110 11589-94

[24] Cazalets J R and Bertrand S 2000 Coupling between lumbar and sacral motor networks in the neonatal rat spinal cord Eur. J. Neurosci. 12 2993-3002

[25] Deliagina T G, Orlovsky G N and Pavlova G A 1983 The Capacity for generation of rhythmic oscillations is distributed in the lumbosacral spinal cord of the cat Exp. Brain Res. 53 81-90

[26] Mortin L I and Stein P S 1989 Spinal cord segments containing key elements of the central pattern generators for three forms of scratch reflex in the turtle. J. Neurosci. 9 2285-96

[27] Daeschler E B, Shubin N H and Jenkins F A 2006 A Devonian tetrapod-like fish and the evolution of the tetrapod body plan Nature 440 757-63

[28] Büschges A, Akay T, Gabriel J P and Schmidt J 2008 Organizing network action for locomotion: Insights from studying insect walking Brain Res. Rev. 57 162-71

[29] Brownstone R M and Wilson J M 2008 Strategies for delineating spinal locomotor rhythm-generating networks and the possible role of $\mathrm{Hb} 9$ interneurones in rhythmogenesis. Brain Res. Rev. 57 64-76

[30] Ivanenko Y P, Poppele R E and Lacquaniti F 2006 Motor control programs and walking Neuroscientist 12 339-48

[31] Hooper S L, Guschlbauer C, Blumel M, Rosenbaum P, Gruhn M, Akay T and Buschges A 2009 Neural Control of Unloaded Leg Posture and of Leg Swing in Stick Insect, Cockroach, and Mouse Differs from That in Larger Animals J. Neurosci. 29 4109-19

[32] Ivanenko Y P, Poppele R E and Lacquaniti F 2004 Five basic muscle activation patterns account for muscle activity during human locomotion J. Physiol. 556 267-82

[33] Grillner S 2011 Control of Locomotion in Bipeds, Tetrapods, and Fish Comprehensive Physiology (Hoboken, NJ, USA: John Wiley \& Sons, Inc.) pp 1179-236

[34] Bellardita C and Kiehn O 2015 Phenotypic characterization of speed-associated gait 
changes in mice reveals modular organization of locomotor networks Curr. Biol. 25 $1426-36$

[35] Endo T and Kiehn O 2008 Asymmetric Operation of the Locomotor Central Pattern Generator in the Neonatal Mouse Spinal Cord J. Neurophysiol. 100 3043-54

[36] Kobayashi R, Nishimaru H and Nishijo H 2016 Estimation of excitatory and inhibitory synaptic conductance variations in motoneurons during locomotor-like rhythmic activity Neuroscience 335 72-81

[37] Shefchyk S J and Jordan L M 1985 Motoneuron input-resistance changes during fictive locomotion produced by stimulation of the mesencephalic locomotor region. $J$. Neurophysiol. 54 1101-8

[38] Orsal D, Perret C and Cabelguen J M 1986 Evidence of rhythmic inhibitory synaptic influences in hindlimb motoneurons during fictive locomotion in the thalamic cat Exp. Brain Res. 64 217-24

[39] Gödderz W, Illert M and Yamaguchi T 1990 Efferent Pattern of Fictive Locomotion in the Cat Forelimb: with Special Reference to Radial Motor Nuclei Eur. J. Neurosci. 2 $663-71$

[40] Burke R E, Degtyarenko A M and Simon E S 2001 Patterns of locomotor drive to motoneurons and last-order interneurons: clues to the structure of the CPG. $J$. Neurophysiol. 86 447-62

[41] Duysens J and Pearson K G 1976 The role of cutaneous afferents from the distal hindlimb in the regulation of the step cycle of thalamic cats Exp. Brain Res. 24 245-55

[42] Duysens J, McCrea D A and Myriam L-R 2006 How deletions in a model could help explain deletions in the laboratory. J. Neurophysiol. 95 562-3; author reply 563-5

[43] Duysens J, De Groote F and Jonkers I 2013 The flexion synergy, mother of all synergies and father of new models of gait Front. Comput. Neurosci. 714

[44] Perret C and Cabelguen J M 1976 Central and reflex participation in the timing of locomotor activations of a bifunctional muscle, the semi-tendinosus, in the cat Brain Res. $106390-5$

[45] Shevtsova N A, Talpalar A E, Markin S N, Harris-Warrick R M, Kiehn O and Rybak I A 2015 Organization of left-right coordination of neuronal activity in the mammalian spinal cord: Insights from computational modelling J. Physiol. 593 2403-26

[46] Molkov Y I, Bacak B J, Talpalar A E and Rybak I A 2015 Mechanisms of Left-Right Coordination in Mammalian Locomotor Pattern Generation Circuits: A Mathematical Modeling View ed J Ayers PLoS Comput. Biol. 11 e1004270

[47] Rybak I A, Dougherty K J and Shevtsova N A 2015 Organization of the Mammalian Locomotor CPG: Review of Computational Model and Circuit Architectures Based on Genetically Identified Spinal Interneurons eNeuro 2 ENEURO.0069-15.2015

[48] Lafreniere-Roula M and McCrea D A 2005 Deletions of Rhythmic Motoneuron Activity During Fictive Locomotion and Scratch Provide Clues to the Organization of the Mammalian Central Pattern Generator J. Neurophysiol. 94 1120-32

[49] Rybak I A, Stecina K, Shevtsova N A and McCrea D A 2006 Modelling spinal circuitry involved in locomotor pattern generation: Insights from the effects of afferent stimulation J. Physiol. 577 641-58 
[50] McCrea D A and Rybak I A 2008 Organization of mammalian locomotor rhythm and pattern generation Brain Res. Rev. 57 134-46

[51] Wilson J M 2005 Conditional Rhythmicity of Ventral Spinal Interneurons Defined by Expression of the Hb9 Homeodomain Protein J. Neurosci. 25 5710-9

[52] Brocard F, Tazerart S and Vinay L 2010 Do pacemakers drive the central pattern generator for locomotion in mammals? Neuroscientist 16 139-55

[53] Brocard F, Shevtsova N A, Bouhadfane M, Tazerart S, Heinemann U, Rybak I A and Vinay L 2013 Activity-Dependent Changes in Extracellular $\mathrm{Ca} 2+$ and $\mathrm{K}+$ Reveal Pacemakers in the Spinal Locomotor-Related Network Neuron 77 1047-54

[54] Ziskind-Conhaim L and Hochman S 2017 Diversity of molecularly-defined spinal interneurons engaged in mammalian locomotor pattern generation J. Neurophysiol. 118 jn.00322.2017

[55] Duysens J 1977 Reflex control of locomotion as revealed by stimulation of cutaneous afferents in spontaneously walking premammillary cats. J. Neurophysiol. 40 737-51

[56] Zhong G, Shevtsova N A, Rybak I A and Harris-Warrick R M 2012 Neuronal activity in the isolated mouse spinal cord during spontaneous deletions in fictive locomotion: insights into locomotor central pattern generator organization. J. Physiol. 590 4735-59

[57] Pearson K G and Duysens J 1976 Function of Segmental Reflexes in the Control of Stepping in Cockroaches and Cats (Springer, Boston, MA) pp 519-37

[58] Danner S M, Wilshin S D, Shevtsova N A and Rybak I A 2016 Central control of interlimb coordination and speed-dependent gait expression in quadrupeds J. Physiol. 594 6947-67

[59] Danner S M, Shevtsova N A, Frigon A and Rybak I A 2017 Computational modeling of spinal circuits controlling limb coordination and gaits in quadrupeds Elife $\mathbf{6}$

[60] Shevtsova N A and Rybak I A 2016 Organization of flexor-extensor interactions in the mammalian spinal cord: insights from computational modelling J. Physiol. 594 6117-31

[61] Ausborn J, Snyder A C, Shevtsova N A, Rybak I A and Rubin J E 2017 State-Dependent Rhythmogenesis and Frequency Control in a Half-Center Locomotor CPG $J$. Neurophysiol. 119 jn.00550.2017

[62] Pearson K G and Iles J F 1970 Discharge patterns of coxal levator and depressor motoneurones of the cockroach, Periplaneta americana. J. Exp. Biol. 52 139-65

[63] Pearson K G 1972 Central programming and reflex control of walking in the cockroach J Exp.Biol. 56 173-93

[64] Fourtner C R 1976 Central nervous control of cockroach walking Neural Control of Locomotion (Springer, Boston, MA) pp 401-18

[65] Zhang J, Lanuza G M, Britz O, Wang Z, Siembab V C, Zhang Y, Velasquez T, Alvarez F J, Frank E and Goulding M 2014 V1 and V2b interneurons secure the alternating flexor-extensor motor activity mice require for limbed locomotion Neuron 82 138-50

[66] Britz O, Zhang J, Grossmann K S, Dyck J, Kim J C, Dymecki S, Gosgnach S and Goulding M 2015 A genetically defined asymmetry underlies the inhibitory control of flexor-extensor locomotor movements Elife 4

[67] Pearson K G 2004 Generating the walking gait: role of sensory feedback. Prog. Brain Res. 143 123-9 
[68] Büschges A and El Manira A 1998 Sensory pathways and their modulation in the control of locomotion Curr. Opin. Neurobiol. 8 733-9

[69] Edwards D H and Prilutsky B I 2017 Sensory Feedback in the Control of Posture and Locomotion Neurobiology of Motor Control (Hoboken, NJ, USA: John Wiley \& Sons, Inc.) pp 263-304

[70] Dietz V and Duysens J 2000 Significance of load receptor input during locomotion: A review Gait Posture 11 102-10

[71] Nichols T R 2018 Distributed force feedback in the spinal cord and the regulation of limb mechanics J. Neurophysiol. 119 1186-200

[72] Duysens J and Pearson K G 1980 Inhibition of flexor burst generation by loading ankle extensor muscles in walking cats Brain Res. 187 321-32

[73] Conway B A, Hultborn H and Kiehn O 1987 Proprioceptive input resets central locomotor rhythm in the spinal cat Exp. Brain Res. 68 643-56

[74] Stephens M J and Yang J F 1996 Short-latency, non-reciprocal group I inhibition is reduced during the stance phase of walking in humans Brain Res. 743 24-31

[75] Faist M, Hoefer C, Hodapp M, Dietz V, Berger W and Duysens J 2006 In humans Ib facilitation depends on locomotion while suppression of $\mathrm{Ib}$ inhibition requires loading. Brain Res. 1076 87-92

[76] Pearson K G, Ramirez J M and Jiang W 1992 Entrainment of the locomotor rhythm by group Ib afferents from ankle extensor muscles in spinal cats Exp. Brain Res. 90 557-66

[77] Pearson K G 1993 Common Principles of Motor Control in Vertebrates and Invertebrates Annu. Rev. Neurosci. 16 265-97

[78] Pearson K G 1995 Proprioceptive regulation of locomotion Curr. Opin. Neurobiol. 5 786-91

[79] Pearson K G, Misiaszek J E and Fouad K 1998 Enhancement and resetting of locomotor activity by muscle afferents Ann. N. Y. Acad. Sci. $860203-15$

[80] Yang J F, Stephens M J and Vishram R 1998 Infant stepping: a method to study the sensory control of human walking J. Physiol. 507 927-37

[81] Pang M Y C and Yang J F 2000 The initiation of the swing phase in human infant stepping: Importance of hip position and leg loading J. Physiol. 528 389-404

[82] Ghori G M U and Luckwill R G 1985 Responses of the lower limb to load carrying in walking man Eur. J. Appl. Physiol. Occup. Physiol. 54 145-50

[83] Grey M J, Mazzaro N, Nielsen J B and Sinkjaer T 2004 Ankle extensor proprioceptors contribute to the enhancement of the soleus EMG during the stance phase of human walking. Can. J. Physiol. Pharmacol. 82 610-6

[84] Cohen A H 1992 The role of heterarchical control in the evolution of central pattern generators Brain Behav. Evol. 40 112-24

[85] Kuo A D 2002 The Relative Roles of Feedforward and Feedback in the Control of Rhythmic Movements Motor Control 6 129-45

[86] Forssberg H 1980 The locomotion of the low spinal cat. I. Coordination within a hindlimb. Acta Physiol Scand 108(3) 269-81

[87] Duysens J, Trippel M, Horstmann G A and Dietz V 1990 Gating and reversal of reflexes 
in ankle muscles during human walking Exp. Brain Res. 82 351-8

[88] Yang J F and Stein R B 1990 Phase-dependent reflex reversal in human leg muscles during walking. J. Neurophysiol. 63 1109-17

[89] Pearson K G and Ramirez J M 1997 Sensory modulation of pattern-generating circuits Neuron, networks, Mot. Behav. 225-35

[90] Sherrington C S 1913 Further observations on the production of reflex stepping by combination of reflex excitation with reflex inhibition J. Physiol. 47 196-214

[91] Pearson K G 2008 Role of sensory feedback in the control of stance duration in walking cats. Brain Res. Rev. 57 222-7

[92] Gossard J P, Cabelguen J M and Rossignol S 1990 Phase-dependent modulation of primary afferent depolarization in single cutaneous primary afferents evoked by peripheral stimulation during fictive locomotion in the cat Brain Res. 537 14-23

[93] Baken B C M, Dietz V and Duysens J 2005 Phase-dependent modulation of short latency cutaneous reflexes during walking in man Brain Res. 1031 268-75

[94] Zehr E P, Komiyama T and Stein R B 1997 Cutaneous reflexes during human gait: electromyographic and kinematic responses to electrical stimulation. J. Neurophysiol. 77 $3311-25$

[95] Côté M-P, Ménard A and Gossard J-P 2003 Spinal cats on the treadmill: changes in load pathways. J. Neurosci. 23 2789-96

[96] Gossard J P, Brownstone R M, Barajon I and Hultborn H 1994 Transmission in a locomotor-related group $\mathrm{Ib}$ pathway from hindlimb extensor muscles in the cat Exp. Brain Res. 98 213-28

[97] McCrea D A, Shefchyk S J, Stephens M J and Pearson K G 1995 Disynaptic group I excitation of synergist ankle extensor motoneurones during fictive locomotion in the cat. J. Physiol. 487 527-39

[98] Whelan P J 1996 Control of locomotion in the decerebrate cat Prog. Neurobiol. 49 481515

[99] Rossignol S, Dubuc R and Gossard J-P 2006 Dynamic Sensorimotor Interactions in Locomotion Physiol. Rev. 86 89-154

[100] Grillner S and Rossignol S 1978 On the initiation of the swing phase of locomotion in chronic spinal cats Brain Res. 146 269-77

[101] GW H, PJ W, Prochazka a, KG. P, Hiebert G W, Whelan P J, Prochazka a and Pearson K G 1996 Contribution of hind limb flexor muscle afferents to the timing of phase transitions in the cat step cycle. J. Neurophysiol. 75 1126-37

[102] Andersson O and Grillner S 1981 Peripheral control of the cat's step cycle I. Phase dependent effects of ramp-movements of the hip during "fictive locomotion" Acta Physiol. Scand. 113 89-101

[103] Andersson O and Grillner S 1983 Peripheral control of the cat's step cycle: II. Entrainment of the central pattern generators for locomotion by sinusoidal hip movements during "fictive locomotion" Acta Physiol. Scand. 118 229-39

[104] Wu M, Gordon K, Kahn J H and Schmit B D 2011 Prolonged electrical stimulation over hip flexors increases locomotor output in human SCI. Clin. Neurophysiol. 122 1421-8 
[105] Akay T, Tourtellotte W G, Arber S and Jessell T M 2014 Degradation of mouse locomotor pattern in the absence of proprioceptive sensory feedback Proc. Natl. Acad. Sci. 111 16877-82

[106] Kiehn O 2016 Decoding the organization of spinal circuits that control locomotion Nat. Rev. Neurosci. 17 224-38

[107] Duysens J and Van De Crommert H W A A 1998 Neural control of locomotion; Part 1: The central pattern generator from cats to humans Gait Posture 7 131-41

[108] Winter D A 1989 Biomechanics of normal and pathological gait: Implications for understanding human locomotor control J. Mot. Behav. 21 337-55

[109] Côté M-P, Murray L M and Knikou M 2018 Spinal Control of Locomotion: Individual Neurons, Their Circuits and Functions Front. Physiol. 9784

[110] Guertin P A 2009 The mammalian central pattern generator for locomotion Brain Res. Rev. 62 45-56

[111] Grillner S, Wallén P, Saitoh K, Kozlov A and Robertson B 2008 Neural bases of goaldirected locomotion in vertebrates--an overview. Brain Res. Rev. 57 2-12

[112] Duysens J, Tax A A, Murrer L and Dietz V 1996 Backward and forward walking use different patterns of phase-dependent modulation of cutaneous reflexes in humans. $J$. Neurophysiol. 76 301-10

[113] Hoogkamer W, Meyns P and Duysens J 2014 Steps forward in understanding backward gait: from basic circuits to rehabilitation. Exerc. Sport Sci. Rev. 42 23-9

[114] Musienko P E, Zelenin P V., Lyalka V F, Gerasimenko Y P, Orlovsky G N and Deliagina T G 2012 Spinal and supraspinal control of the direction of stepping during locomotion. J. Neurosci. 32 17442-53

[115] Shik M L, Severin F V and Orlovskiǔ G N 1966 [Control of walking and running by means of electric stimulation of the midbrain]. Biofizika 11 659-66

[116] Martinez-Gonzalez C, Wang H-L, Micklem B R, Bolam J P and Mena-Segovia J 2012 Subpopulations of cholinergic, GABAergic and glutamatergic neurons in the pedunculopontine nucleus contain calcium-binding proteins and are heterogeneously distributed. Eur. J. Neurosci. 35 723-34

[117] Caggiano V, Leiras R, Goñi-Erro H, Masini D, Bellardita C, Bouvier J, Caldeira V, Fisone $\mathrm{G}$ and Kiehn $\mathrm{O} 2018$ Midbrain circuits that set locomotor speed and gait selection. Nature 553 455-60

[118] Roseberry T K, Lee A M, Lalive A L, Wilbrecht L, Bonci A and Kreitzer A C 2016 Cell-Type-Specific Control of Brainstem Locomotor Circuits by Basal Ganglia. Cell 164 526-37

[119] Josset N, Roussel M, Lemieux M, Lafrance-Zoubga D, Rastqar A and Bretzner F 2018 Distinct Contributions of Mesencephalic Locomotor Region Nuclei to Locomotor Control in the Freely Behaving Mouse. Curr. Biol. 28 884-901.e3

[120] Esposito M S and Arber S 2016 Motor Control: Illuminating an Enigmatic Midbrain Locomotor Center. Curr. Biol. 26 R291-3

[121] Davis M, Gendelman D S, Tischler M D and Gendelman P M 1982 A primary acoustic startle circuit: lesion and stimulation studies. J. Neurosci. 2 791-805

[122] Davis M 1989 Neural systems involved in fear-potentiated startle. Ann. N. Y. Acad. Sci. 


\section{$563165-83$}

[123] Simons-Weidenmaier N S, Weber M, Plappert C F, Pilz P K D and Schmid S 2006 Synaptic depression and short-term habituation are located in the sensory part of the mammalian startle pathway BMC Neurosci. 738

[124] Sherman D, Fuller P M, Marcus J, Yu J, Zhang P, Chamberlin N L, Saper C B and Lu J 2015 Anatomical location of the mesencephalic locomotor region and its possible role in locomotion, posture, cataplexy, and Parkinsonism Front. Neurol. 6140

[125] Roseberry T K and Kreitzer A 2017 Neural circuitry for behavioural arrest. Philos. Trans. R. Soc. Lond. B. Biol. Sci. 37220160197

[126] Nieuwenhuijzen P H, Schillings A M, Van Galen G P and Duysens J 2000 Modulation of the startle response during human gait. J. Neurophysiol. 84 65-74

[127] Bouvier J, Caggiano V, Leiras R, Caldeira V, Bellardita C, Balueva K, Fuchs A and Kiehn O 2015 Descending Command Neurons in the Brainstem that Halt Locomotion Cell 163 1191-203

[128] Giber K, Diana M A, M Plattner V, Dugué G P, Bokor H, Rousseau C V., Maglóczky Z, Havas L, Hangya B, Wildner H, Zeilhofer H U, Dieudonné S and Acsády L 2015 A subcortical inhibitory signal for behavioral arrest in the thalamus Nat. Neurosci. $18562-$ 8

[129] Juvin L, Grätsch S, Trillaud-Doppia E, Gariépy J F, Büschges A and Dubuc R 2016 A Specific Population of Reticulospinal Neurons Controls the Termination of Locomotion Cell Rep. 15 2377-86

[130] Mullins O J, Hackett J T, Buchanan J T and Friesen W O 2011 Neuronal control of swimming behavior: Comparison of vertebrate and invertebrate model systems Prog. Neurobiol. $93244-69$

[131] Mantyh P W 1983 Connections of midbrain periaqueductal gray in the monkey. II. Descending efferent projections. J. Neurophysiol. 49 582-94

[132] Robinson T E 1978 Electrical stimulation of the brain stem in freely moving rats: I. Effects on behavior. Physiol. Behav. 21 223-31

[133] Renner R and Behnke S 2006 Instability detection and fall avoidance for a humanoid using attitude sensors and reflexes IEEE International Conference on Intelligent Robots and Systems (IEEE) pp 2967-73

[134] Brown T G 1914 On the nature of the fundamental activity of the nervous centres; together with an analysis of the conditioning of rhythmic activity in progression, and a theory of the evolution of function in the nervous system J. Physiol. 48 18-46

[135] Grillner S 2006 Biological Pattern Generation: The Cellular and Computational Logic of Networks in Motion Neuron 52 751-66

[136] Taga G 1995 A model of the neuro-musculo-skeletal system for human locomotion - I. Emergence of basic gait Biol. Cybern. 73 97-111

[137] Verdaasdonk B W, Koopman H F J M and Helm F C T Van Der 2006 Energy efficient and robust rhythmic limb movement by central pattern generators. Neural Netw. 19388 400

[138] Hase Kazunori and Yamazaki N 2002 Computer Simulation Study of Human Locomotion with a Three-Dimensional Entire-Body Neuro-Musculo-Skeletal Model. I. 
Acquisition of Normal Walking. JSME Int. J. Ser. C 45 1040-50

[139] Ijspeert A J 2008 Central pattern generators for locomotion control in animals and robots: A review Neural Networks 21 642-53

[140] Klein T J and Lewis M A 2012 A physical model of sensorimotor interactions during locomotion J. Neural Eng. 9046011

[141] Kim Y, Tagawa Y, Obinata G and Hase K 2011 Robust control of CPG-based 3D neuromusculoskeletal walking model Biol. Cybern. 105 269-82

[142] Vogelstein R J, Tenore F, Etienne-Cummings R, Lewis M A and Cohen A H 2006 Dynamic control of the central pattern generator for locomotion Biol. Cybern. 95 55566

[143] Mazurek K A, Holinski B J, Everaert D G, Stein R B, Etienne-Cummings R and Mushahwar V K 2012 Feed forward and feedback control for over-ground locomotion in anaesthetized cats J. Neural Eng. 9026003

[144] Endo G, Nakanishi J, Morimoto J and Cheng G 2005 Experimental studies of a neural oscillator for biped locomotion with QRIO Proceedings - IEEE International Conference on Robotics and Automation vol 2005 (IEEE) pp 596-602

[145] Righetti L and Auke Jan Ijspeert Programmable central pattern generators: an application to biped locomotion control Proceedings 2006 IEEE International Conference on Robotics and Automation, 2006. ICRA 2006. (IEEE) pp 1585-90

[146] Morimoto J, Endo G, Nakanishi J, Hyon S H, Cheng G, Bentivegna D and Atkeson C G 2006 Modulation of simple sinusoidal patterns by a coupled oscillator model for biped walking Proceedings - IEEE International Conference on Robotics and Automation vol 2006 (IEEE) pp 1579-84

[147] Matsuoka K 1985 Sustained oscillations generated by mutually inhibiting neurons with adaptation Biol. Cybern. 52 367-76

[148] Van Der Noot N, Ijspeert A J and Ronsse R 2015 Biped gait controller for large speed variations, combining reflexes and a central pattern generator in a neuromuscular model Proceedings - IEEE International Conference on Robotics and Automation vol 2015June (IEEE) pp 6267-74

[149] Dzeladini F, van den Kieboom J and Ijspeert A 2014 The contribution of a central pattern generator in a reflex-based neuromuscular model Front. Hum. Neurosci. 8371

[150] Geyer H and Herr H 2010 A Muscle-reflex model that encodes principles of legged mechanics produces human walking dynamics and muscle activities IEEE Trans. Neural Syst. Rehabil. Eng. 18 263-73

[151] Geng T, Porr B and Wörgötter F 2006 Fast biped walking with a sensor-driven neuronal controller and real-time online learning Int. J. Rob. Res. 25 243-59

[152] Manoonpong P, Geng T, Kulvicius T, Porr B and Wörgötter F 2007 Adaptive, fast walking in a biped robot under neuronal control and learning PLoS Comput. Biol. 3 1305-20

[153] Song S and Geyer H 2017 Evaluation of a Neuromechanical Walking Control Model Using Disturbance Experiments. Front. Comput. Neurosci. 1115

[154] Taga G 1995 A model of the neuro-musculo-skeletal system for human locomotion - II. Real-time adaptability under various constraints Biol. Cybern. 73 113-21 
[155] Abbas J J and Full R J 2000 Neuromechanical Interaction of Cyclic Movements Biomechanics and Neural Control of Posture and Movement ed J M Winters and P E Crago (New York: Springer-Verlag New York, Inc.) pp 177-91

[156] Klavins E, Komsuoglu H, Full R J and Koditschek D E 2002 The Role of Reflexes Versus Central Pattern Generators in Dynamical Legged Locomotion Neurotechnology Biomim. Robot. 351-82

[157] Wu Q Di, Liu C J, Zhang J Q and Chen Q J 2009 Survey of locomotion control of legged robots inspired by biological concept Sci. China, Ser. F Inf. Sci. 52 1715-29

[158] Crespi A, Lachat D, Pasquier A and Ijspeert A J 2008 Controlling swimming and crawling in a fish robot using a central pattern generator Auton. Robots 25 3-13

[159] Rinderknecht M D, Delaloye F A, Crespi A, Ronsse R and Ijspeert A J 2011 Assistance using adaptive oscillators: Robustness to errors in the identification of the limb parameters IEEE International Conference on Rehabilitation Robotics (IEEE) pp 1-6

[160] Taga G 1998 A model of the neuro-musculo-skeletal system for anticipatory adjustment of human locomotion during obstacle avoidance. Biol. Cybern. 78 9-17

[161] Fukuoka Y, Kimura H and Cohen A H 2003 Adaptive Dynamic Walking of a Quadruped Robot on Irregular Terrain Based on Biological Concepts Int. J. Rob. Res. 22 187-202

[162] Matsuoka K 1987 Mechanisms of frequency and pattern control in the neural rhythm generators Biol. Cybern. 56 345-53

[163] Aoi S and Tsuchiya K 2005 Locomotion control of a biped robot using nonlinear oscillators Auton. Robots 19 219-32

[164] Morimoto J, Endo G, Nakanishi J and Cheng G 2008 A biologically inspired biped locomotion strategy for humanoid robots: Modulation of sinusoidal patterns by a coupled oscillator model IEEE Trans. Robot. 24 185-91

[165] Wright J and Jordanov I 2015 Intelligent Approaches in Locomotion - A Review J. Intell. Robot. Syst. Theory Appl. 80 255-77

[166] Nichols R and Ross K T 2009 The Implications of Force Feedback for the $\lambda$ Model Adv. Exp. Med. Biol. $629663-79$

[167] Jo S and Massaquoi S G 2007 A model of cerebrocerebello-spinomuscular interaction in the sagittal control of human walking Biol. Cybern. 96 279-307

[168] Jo S 2008 Hypothetical neural control of human bipedal walking with voluntary modulation Med. Biol. Eng. Comput. 46 179-93

[169] Yang J F, Stein R B and James K B 1991 Contribution of peripheral afferents to the activation of the soleus muscle during walking in humans Exp. Brain Res. 87 679-87

[170] af Klint R, Mazzaro N, Nielsen J B, Sinkjaer T and Grey M J 2010 Load rather than length sensitive feedback contributes to soleus muscle activity during human treadmill walking. J. Neurophysiol. $1032747-56$

[171] Sinkjaer T, Andersen J B, Ladouceur M, Christensen L O and Nielsen J B 2000 Major role for sensory feedback in soleus EMG activity in the stance phase of walking in man. J. Physiol. 523 Pt 3 817-27

[172] Mazzaro N, Grey M J and Sinkjaer T 2005 Contribution of afferent feedback to the soleus muscle activity during human locomotion J. Neurophysiol. 93 167-77 
[173] Ekeberg Ö and Pearson K 2005 Computer Simulation of Stepping in the Hind Legs of the Cat: An Examination of Mechanisms Regulating the Stance-to-Swing Transition $J$. Neurophysiol. 94 4256-68

[174] Prochazka A, Gillard D and Bennett D J 1997 Positive Force Feedback Control of Muscles J. Neurophysiol. 77 3226-36

[175] Fukuoka Y, Fukino K, Habu Y and Mori Y 2015 Energy evaluation of a bio-inspired gait modulation method for quadrupedal locomotion Bioinspiration and Biomimetics 10 046017

[176] Verdaasdonk B W, Koopman H F J M and Van Der Helm F C T 2009 Energy efficient walking with central pattern generators: From passive dynamic walking to biologically inspired control Biol. Cybern. 101 49-61

[177] Ogihara N and Yamazaki N 2001 Generation of human bipedal locomotion by a biomimetic neuro-musculo-skeletal model Biol. Cybern. 84 1-11

[178] Paul C, Bellotti M, Jezernik S and Curt A 2005 Development of a human neuromusculo-skeletal model for investigation of spinal cord injury Biol. Cybern. 93 153-70

[179] Song S and Geyer H 2015 A neural circuitry that emphasizes spinal feedback generates diverse behaviours of human locomotion J. Physiol. 593 3493-511

[180] Maufroy C, Kimura H and Takase K 2008 Towards a general neural controller for 3D quadrupedal locomotion Proc. SICE Annu. Conf. 21 2495-500

[181] Feldman A G 1966 On the functional tuning of the nervous system in movement control or preservation of stationary pose. II. Adjustable parameters in muscles Biofizika 11 498508

[182] Kimura H, Fukuoka Y and Nakamura H 1999 Biologically Inspired Adaptive Dynamic Walking of the Quadruped on Irregular Terrain Proc. of 9th Intl Symposium of Robotics Research (ISRR'99), held in Snowbird vol 25 (London: Springer London) pp 271-8

[183] Aoi S, Ogihara N, Funato T and Tsuchiya K 2012 Sensory regulation of stance-to-swing transition in generation of adaptive human walking: A simulation study Rob. Auton. Syst. 60 685-91

[184] Forner-Cordero A, Koopman H J F M and Van Der Helm F C T 2006 Describing gait as a sequence of states J. Biomech. 39 948-57

[185] Sharbafi M A and Seyfarth A 2017 How locomotion sub-functions can control walking at different speeds? J. Biomech. 53 163-70

[186] Ekeberg Ö and Wadden T 1998 A neuro-mechanical model of legged locomotion: single leg control Biol. Cybern. 79 161-73

[187] Molkov Y I, Rubin J E, Rybak I A and Smith J C 2017 Computational models of the neural control of breathing Wiley Interdiscip. Rev. Syst. Biol. Med. 9

[188] McVea D A and Pearson K G 2009 Object avoidance during locomotion Adv. Exp. Med. Biol. 629 293-315

[189] Loeb G E and Duysens J 1979 Activity patterns in individual hindlimb primary and secondary muscle spindle afferents during normal movements in unrestrained cats. $J$. Neurophysiol. 42 420-40

[190] Prochazka a, Westerman R a and Ziccone S P 1976 Discharges of single hindlimb afferents in the freely moving cat. J. Neurophysiol. 39 1090-104 
[191] Faist M, Blahak C, Duysens J and Berger W 1999 Modulation of the biceps femoris tendon jerk reflex during human locomotion Exp. Brain Res. 125 265-70

[192] Duysens J, van Wezel B M, van de Crommert H W, Faist M and Kooloos J G 1998 The role of afferent feedback in the control of hamstrings activity during human gait. Eur. J. Morphol. 36 293-9

[193] Sharbafi M A, Rode C, Kurowski S, Scholz D, Möckel R, Radkhah K, Zhao G, Rashty A M, Stryk O von and Seyfarth A 2016 A new biarticular actuator design facilitates control of leg function in BioBiped3 Bioinspir. Biomim. 11046003

[194] Cruse H, Kindermann T, Schumm M, Dean J and Schmitz J 1998 Walknet - A biologically inspired network to control six-legged walking Neural Networks 11 143547

[195] Duysens J, Bastiaanse C M, Smits-Engelsman B C . and Dietz V 2004 Gait acts as a gate for reflexes from the foot Can. J. Physiol. Pharmacol. 82 715-22

[196] Zehr E P and Duysens J 2004 Regulation of arm and leg movement during human locomotion Neuroscientist 10 347-61

[197] Capaday C and Stein R B 1986 Amplitude modulation of the soleus H-reflex in the human during walking and standing. J. Neurosci. 6 1308-13

[198] Forssberg H, Grillner S and Rossignol S 1975 Phase dependent reflex reversal during walking in chronic spinal cats Brain Res. 85 103-7

[199] Duysens J and Loeb G E 1980 Modulation of ipsi- and contralateral reflex responses in unrestrained walking cats. J. Neurophysiol. 44 1024-37

[200] Van Wezel B M, Ottenhoff F a and Duysens J 1997 Dynamic control of location-specific information in tactile cutaneous reflexes from the foot during human walking. $J$. Neurosci. 17 3804-14

[201] Szczecinski N S, Hunt A J and Quinn R D 2017 Design process and tools for dynamic neuromechanical models and robot controllers Biol. Cybern. 111 105-27

[202] Wright J and Jordanov I 2015 Intelligent approaches in locomotion-a review J. Intell. Robot. Syst. $80255-77$

[203] Vukobratovic M and Juricic D 1969 Contribution to the Synthesis of Biped Gait IEEE Trans. Biomed. Eng. BME-16 1-6

[204] Vukobratovic M and Borovac B 2004 Zero-Moment Point - Thirty Five Years of its Life. I. J. Humanoid Robot. 1 157-73

[205] Collins S, Ruina A, Tedrake R and Wisse M 2005 Efficient bipedal robots based on passive-dynamic walkers Science (80-. ). 307 1082-5

[206] Pratt J, Carff J, Drakunov S and Goswami A 2006 Capture Point: A Step toward Humanoid Push Recovery Humanoid Robots, 2006 6th IEEE-RAS International Conference on pp 200-7

[207] Pratt J E, Koolen T, de Boer T, Rebula J R, Cotton S, Carff J, Johnson M and Neuhaus P D 2012 Capturability-based analysis and control of legged locomotion, Part 2: Application to M2V2, a lower-body humanoid. I. J. Robot. Res. 31 1117-33

[208] Vukobratovic M and Stepanenko J 1972 On the stability of anthropomorphic systems Math. Biosci. 15 1-37 
[209] Takanishi A, Ishida M, Yamazaki Y and Kato I 1985 The Realization of Dynamic Walking by the Biped Walking Robot WL10RD Adv. Robot.

[210] Kajita S, Nagasaki T, Yokoi K, Kaneko K and Tanie K 2002 Running pattern generation for a humanoid robot Proceedings-IEEE International Conference on Robotics and Automation vol 3 (IEEE) pp 2755-61

[211] Kajita S, Kanehiro F, Kaneko K, Fujiwara K, Harada K, Yokoi K H K and Hirukawa H 2003 Biped walking pattern generation by using preview control of zero-moment point in Proceedings of the IEEE International Conference on Robotics and Automation vol 2 pp 1620-6

[212] Feng S, Whitman E, Xinjilefu X and Atkeson C G 2014 Optimization based full body control for the atlas robot 2014 IEEE-RAS International Conference on Humanoid Robots pp 120-7

[213] Tedrake R, Kuindersma S, Deits R and Miura K 2015 A closed-form solution for realtime ZMP gait generation and feedback stabilization Humanoid Robots (Humanoids), 2015 IEEE-RAS 15th International Conference on pp 936-40

[214] Vukobratovic M, Borovac B and Potkonjak V 2006 Zmp: a Review of Some Basic Misunderstandings I. J. Humanoid Robot. 3 153-75

[215] Dingwell J B, Cusumano J P, Cavanagh P R and Sternad D 2001 Local Dynamic Stability Versus Kinematic Variability of Continuous Overground and Treadmill Walking J. Biomech. Eng. 12327

[216] Hausdorff J M 2005 Gait variability: Methods, modeling and meaning J. Neuroeng. Rehabil. 219

[217] McGeer T 1990 Passive walking with knees Robotics and Automation, 1990. Proceedings., 1990 IEEE International Conference on pp 1640-5 vol.3

[218] Collins S H S H and Ruina A 2005 A bipedal walking robot with efficient and humanlike gait Proceedings of the 2005 IEEE international conference on robotics and automation (IEEE) pp 1983-8

[219] Hof A L 2008 The "extrapolated center of mass" concept suggests a simple control of balance in walking Hum. Mov. Sci. 27 112-25

[220] Pratt J E and Tedrake R 2006 Velocity-Based Stability Margins for Fast Bipedal Walking Fast Motions in Biomechanics and Robotics: Optimization and Feedback Control (Berlin, Heidelberg: Springer Berlin Heidelberg) pp 299-324

[221] Koolen T, De Boer T, Rebula J, Goswami A and Pratt J 2012 Capturability-based analysis and control of legged locomotion, Part 1: Theory and application to three simple gait models Int. J. Rob. Res. 31 1094-113

[222] Krause M, Englsberger J, Wieber P B and Ott C 2012 Stabilization of the Capture Point dynamics for bipedal walking based on model predictive control IFAC Proceedings Volumes (IFAC-PapersOnline) vol 45 (Elsevier) pp 165-71

[223] Townsend M A 1985 Biped gait stabilization via foot placement. J. Biomech. 18 21-38

[224] Forner Cordero A, Koopman H and van der Helm F C T 2004 Mechanical model of the recovery from stumbling Biol. Cybern. $91212-20$

[225] Hof A L, Gazendam M G J and Sinke W E 2005 The condition for dynamic stability J. Biomech. 38 1-8 
[226] Takenaka T, Matsumoto T and Yoshiike T 2009 Real time motion generation and control for biped robot $-1^{\text {st }}$ report: Walking gait pattern generation- 2009 IEEE/RSJ International Conference on Intelligent Robots and Systems (IEEE) pp 1084-91

[227] Englsberger J, Ott C and Albu-Schaffer A 2015 Three-Dimensional Bipedal Walking Control Based on Divergent Component of Motion IEEE Trans. Robot. 31 355-68

[228] Zhang T, Tran M and Huang H 2018 Design and experimental verification of hip exoskeleton with balance capacities for walking assistance IEEE/ASME Trans. Mechatronics 23 274-85

[229] Griffin R J, Wiedebach G, Bertrand S, Leonessa A and Pratt J 2017 Walking stabilization using step timing and location adjustment on the humanoid robot, Atlas 2017 IEEE/RSJ International Conference on Intelligent Robots and Systems (IROS) (IEEE) pp 667-73

[230] Jeong H, Sim O, Bae H, Lee K, Oh J and Oh J-H 2017 Biped walking stabilization based on foot placement control using capture point feedback 2017 IEEE/RSJ International Conference on Intelligent Robots and Systems (IROS) (IEEE) pp 5263-9

[231] Matthis J S and Fajen B R 2013 Visual control of foot placement when walking over complex terrain.

[232] Rossi L F, Rodrigues S T and Forner-Cordero A 2014 Do humans walk humans like robots when crossing an obstacle without visual information? Biomedical Robotics and Biomechatronics, 2014. (BIOROB 2014). Proceedings. 5th IEEE RAS \& EMBS International Conference on (IEEE) pp 216-20

[233] Schillings A M, Van Wezel B M H, Mulder T H and Duysens J 1999 Widespread shortlatency stretch reflexes and their modulation during stumbling over obstacles Brain Res. $816480-6$

[234] Forner-Cordero A, Van Der Helm F C T, Koopman H F J M and Duysens J 2015 Recovery response latencies to tripping perturbations during gait decrease with practice Proceedings of the Annual International Conference of the IEEE Engineering in Medicine and Biology Society, EMBS vol 2015-Novem (IEEE) pp 6748-51

[235] Englsberger J, Ott C, Roa M A, Albu-Schäffer A and Hirzinger G 2011 Bipedal walking control based on capture point dynamics IEEE International Conference on Intelligent Robots and Systems (IEEE) pp 4420-7

[236] Stephens B J 2011 Push recovery control for force-controlled humanoid robots (Carnegie Mellon University Pittsburgh, Pennsylvania USA)

[237] von Holst E and Mittelstaedt H 1950 Das Reafferenzprinzip - Wechselwirkungen zwischen Zentralnervensystem und Peripherie Naturwissenschaften 37 464-76

[238] Holst E and Mittelstaedt H 1971 The principle of reafference: Interactions between the central nervous system and the peripheral organs PC Dodwell (Ed. and Trans.), Perceptual processing: Stimulus equivalence and pattern recognition ed P.C.Dodwell (New York: Appleton-Century-Crofts) pp 41-72

[239] Manoonpong P and Wörgötter F 2009 Efference copies in neural control of dynamic biped walking Rob. Auton. Syst. 57 1140-53

[240] Schröder-Schetelig J, Manoonpong P and Wörgötter F 2010 Using efference copy and a forward internal model for adaptive biped walking Auton. Robots 29 357-66 
[241] Patla A E, Prentice S D, Robinson C and Neufeld J 1991 Visual control of locomotion: Strategies for changing direction and for going over obstacles. J. Exp. Psychol. Hum. Percept. Perform. 17 603-34

[242] Patla A E and Vickers J N 2003 How far ahead do we look when required to step on specific locations in the travel path during locomotion? Exp. Brain Res. 148 133-8

[243] Patla A E and Greig M 2006 Any way you look at it, successful obstacle negotiation needs visually guided on-line foot placement regulation during the approach phase Neurosci. Lett. 397 110-4

[244] Marigold D S and Patla A E 2007 Gaze fixation patterns for negotiating complex ground terrain Neuroscience 144 302-13

[245] Mohagheghi A A, Moraes R and Patla A E 2004 The effects of distant and on-line visual information on the control of approach phase and step over an obstacle during locomotion Exp. Brain Res. 155 459-68

[246] Marigold D S and Patla A E 2008 Visual information from the lower visual field is important for walking across multi-surface terrain Exp. Brain Res. 188 23-31

[247] Marigold D S, Weerdesteyn V, Patla A E and Duysens J 2007 Keep looking ahead? Redirection of visual fixation does not always occur during an unpredictable obstacle avoidance task Exp. Brain Res. 176 32-42

[248] Weerdesteyn V, Nienhuis B, Hampsink B and Duysens J 2004 Gait adjustments in response to an obstacle are faster than voluntary reactions Hum. Mov. Sci. 23 351-63

[249] Rhea C K and Rietdyk S 2007 Visual exteroceptive information provided during obstacle crossing did not modify the lower limb trajectory Neurosci. Lett. 418 60-5

[250] Timmis M A and Buckley J G 2012 Obstacle crossing during locomotion: Visual exproprioceptive information is used in an online mode to update foot placement before the obstacle but not swing trajectory over it Gait Posture 36 160-2

[251] Patla A E, Davies T C and Niechwiej E 2004 Obstacle avoidance during locomotion using haptic information in normally sighted humans Exp. brain Res. 155 173-85

[252] Forner-Cordero A, Garcia V D V.D., Rodrigues S T S T and Duysens J 2016 Obstacle Crossing Differences Between Blind and Blindfolded Subjects After Haptic Exploration J. Mot. Behav. 48 1-11

[253] Potocanac Z and Duysens J 2017 Online adjustments of leg movements in healthy young and old Exp. Brain Res. 235 2329-48

[254] Muller R, Ernst M and Blickhan R 2012 Leg adjustments during running across visible and camouflaged incidental changes in ground level J. Exp. Biol. 215 3072-9

[255] Muller R, Haufle D F B and Blickhan R 2015 Preparing the leg for ground contact in running: the contribution of feed-forward and visual feedback J. Exp. Biol. 218 451-7

[256] Ernst M, Götze M, Müller R and Blickhan R 2014 Vertical adaptation of the center of mass in human running on uneven ground Hum. Mov. Sci. 38 293-304

[257] Daley M A 2008 Biomechanics: Running Over Uneven Terrain Is a No-Brainer Curr. Biol. 18 R1064-6

[258] Hobbelen D G E and Wisse M 2007 A disturbance rejection measure for limit cycle walkers: The gait sensitivity norm IEEE Trans. Robot. 23 1213-24 
[259] Westervelt E R, Grizzle J W, Chevallereau C, Choi J H and Morris B 2007 Feedback Control of Dynamic Bipedal Robot Locomotion vol 26 (CRC Press)

[260] Blickhan R, Andrada E, Müller R, Rode C and Ogihara N 2015 Positioning the hip with respect to the COM: Consequences for leg operation J. Theor. Biol. 382 187-97

[261] McFadyen B J, Winter D A and Allard F 1994 Simulated control of unilateral, anticipatory locomotor adjustments during obstructed gait Biol. Cybern. 72 151-60

[262] Ivanenko Y P, Cappellini G, Dominici N, Poppele R E and Lacquaniti F 2007 Modular Control of Limb Movements during Human Locomotion J. Neurosci. 27 11149-61

[263] Hirai K, Hirose M, Haikawa Y and Takenaka T 1998 The development of Honda humanoid robot Proceedings. 1998 IEEE International Conference on Robotics and Automation (Cat. No.98CH36146) vol 2 (IEEE) pp 1321-6

[264] van der Linden M H, Marigold D S, Gabreels F J M and Duysens J 2007 Muscle Reflexes and Synergies Triggered by an Unexpected Support Surface Height During Walking J. Neurophysiol. 97 3639-50

[265] Shinya M and Oda S 2010 Fast muscle responses to an unexpected foot-in-hole scenario, evoked in the context of prior knowledge of the potential perturbation Exp. Brain Res. $203437-46$

[266] Shinya M, Fujii S and Oda S 2009 Corrective postural responses evoked by completely unexpected loss of ground support during human walking Gait Posture 29 483-7

[267] Frost R, Skidmore J, Santello M and Artemiadis P 2015 Sensorimotor control of gait: a novel approach for the study of the interplay of visual and proprioceptive feedback Front. Hum. Neurosci. 914

[268] van der Linden M H, Hendricks H T, Bloem B R and Duysens J 2009 Hitting a support surface at unexpected height during walking induces loading transients Gait Posture 29 $255-60$

[269] Daley M A and Biewener A A 2006 Running over rough terrain reveals limb control for intrinsic stability Proc. Natl. Acad. Sci. 103 15681-6

[270] Park J H and Kim E S 2009 Foot and body control of biped robots to walk on irregularly protruded uneven surfaces IEEE Trans. Syst. Man, Cybern. Part B Cybern. 39 289-97

[271] Shih C L 1999 Ascending and descending stairs for a biped robot IEEE Trans. Syst. Man, Cybern. Part ASystems Humans. 29 255-68

[272] Figliolini G, Ceccarelli M and Di Gioia M 2003 Descending stairs with EP-WAR3 biped robot IEEE/ASME International Conference on Advanced Intelligent Mechatronics, AIM vol 2 (IEEE) pp 747-52

[273] Kim E S, Kim M S, Choi J Y, Kim S W and Kim J W 2010 Trajectory generation schemes for bipedal ascending and descending stairs using univariate dynamic encoding algorithm for searches (uDEAS) Int. J. Control. Autom. Syst. 8 1061-71

[274] Fu C and Chen K 2008 Gait synthesis and sensory control of stair climbing for a humanoid robot IEEE Trans. Ind. Electron. 55 2111-20

[275] Dietz V, Quintern J, Boos G and Berger W 1986 Obstruction of the swing phase during gait: phase-dependent bilateral leg muscle coordination Brain Res. 384 166-9

[276] Soares S C 2012 The lurking snake in the grass: Interference of snake stimuli in visually taxing conditions Evol. Psychol. 10 187-97 
[277] Soares S C, Lindström B, Esteves F and Öhman A 2014 The hidden snake in the grass: Superior detection of snakes in challenging attentional conditions ed H Nishijo PLoS One 9 e 114724

[278] Soares S C, Maior R S, Isbell L A, Tomaz C and Nishijo H 2017 Fast detector/first responder: Interactions between the superior colliculus-pulvinar pathway and stimuli relevant to primates Front. Neurosci. 1167

[279] Eng J J, Winter D A and Patla A E 1994 Strategies for recovery from a trip in early and late swing during human walking Exp. Brain Res. 102 339-49

[280] Schillings A M, van Wezel B M, Mulder T and Duysens J 2000 Muscular responses and movement strategies during stumbling over obstacles. J. Neurophysiol. 83 2093-102

[281] Schillings A M 2005 Stumbling Over Obstacles in Older Adults Compared to Young Adults J. Neurophysiol. 94 1158-68

[282] Forner Cordero A, Koopman H F J M F and Van der Helm F C T C T 2003 Multiplestep strategies to recover from stumbling perturbations Gait Posture 18 47-59

[283] Forner Cordero A, Koopman H and van der Helm F C T 2005 Energy analysis of human stumbling: the limitations of recovery Gait Posture 21 243-54

[284] Roos P E, McGuigan M P and Trewartha G 2010 The role of strategy selection, limb force capacity and limb positioning in successful trip recovery Clin. Biomech. 25 873-8

[285] Shirota C, Simon A M, Rouse E J and Kuiken T A 2011 The effect of perturbation onset timing and length on tripping recovery strategies Proc. Annu. Int. Conf. IEEE Eng. Med. Biol. Soc. EMBS $20117833-6$

[286] Patla A E and Rietdyk S 1993 Visual control of limb trajectory over obstacles during locomotion: effect of obstacle height and width Gait Posture 1 45-60

[287] de Boer T, Wisse M and der Helm F C T 2010 Mechanical analysis of the preferred strategy selection in human stumble recovery J. Biomech. Eng. 13271012

[288] Pijnappels M, Bobbert M F and Van Dieën J H 2005 Push-off reactions in recovery after tripping discriminate young subjects, older non-fallers and older fallers Gait Posture 21 388-94

[289] Forner-Cordero A, Ackermann M and de Lima Freitas M 2011 A method to simulate motor control strategies to recover from perturbations: Application to a stumble recovery during gait Proceedings of the Annual International Conference of the IEEE Engineering in Medicine and Biology Society, EMBS vol 2011 (IEEE) pp 7829-32

[290] Klemetti R, Moilanen P, Avela J and Timonen J 2014 Effects of gait speed on stability of walking revealed by simulated response to tripping perturbation Gait Posture 39534 9

[291] Pavol M J, Owings T M, Foley K T and Grabiner M D 2001 Mechanisms leading to a fall from an induced trip in healthy older adults. J. Gerontol. A. Biol. Sci. Med. Sci. 56 M428-37

[292] Van den Bogert A J, Pavol M J and Grabiner M D 2002 Response time is more important than walking speed for the ability of older adults to avoid a fall after a trip J. Biomech. 35 199-205

[293] Shinya M, Kawashima N and Nakazawa K 2016 Temporal, but not Directional, Prior Knowledge Shortens Muscle Reflex Latency in Response to Sudden Transition of 
Support Surface During Walking Front. Hum. Neurosci. 1029

[294] Jo S 2007 A neurobiological model of the recovery strategies from perturbed walking BioSystems 90 750-68

[295] Drew T 1991 Functional organization within the medullary reticular formation of the intact unanesthetized cat. III. Microstimulation during locomotion. J. Neurophysiol. 66 919-38

[296] Tomita N and Yano M 2006 Real-time Control of Bipedal Movement based on Basal ganglia and Brainstem systems 2006 SICE-ICASE International Joint Conference (IEEE) pp 4499-502

[297] Mori S 1987 Integration of posture and locomotion in acute decerebrate cats and in awake, freely moving cats Prog. Neurobiol. 28 161-95

[298] Ivanenko Y P 2005 Coordination of Locomotion with Voluntary Movements in Humans J. Neurosci. 25 7238-53

[299] Ivanenko Y P, Cappellini G, Poppele R E and Lacquaniti F 2008 Spatiotemporal organization of ??-motoneuron activity in the human spinal cord during different gaits and gait transitions Eur. J. Neurosci. 27 3351-68

[300] Dominici N, Ivanenko Y P, Cappellini G, D’Avella A, Mondi V, Cicchese M, Fabiano A, Silei T, Di Paolo A, Giannini C, Poppele R E and Lacquaniti F 2011 Locomotor Primitives in Newborn Babies and Their Development Science (80-. ). 334 997-9

[301] Meyer A J, Eskinazi I, Jackson J N, Rao A V., Patten C and Fregly B J 2016 Muscle Synergies Facilitate Computational Prediction of Subject-Specific Walking Motions Front. Bioeng. Biotechnol. 4

[302] Jo S and Massaquoi S G 2004 A model of cerebellum stabilized and scheduled hybrid long-loop control of upright balance Biol. Cybern. 91 188-202

[303] Aoi S and Funato T 2016 Neuromusculoskeletal models based on the muscle synergy hypothesis for the investigation of adaptive motor control in locomotion via sensorymotor coordination. Neurosci. Res. 104 88-95

[304] Marigold D S and Patla A E 2002 Strategies for Dynamic Stability During Locomotion on a Slippery Surface: Effects of Prior Experience and Knowledge J. Neurophysiol. 88 339-53

[305] Marigold D S, Bethune A J and Patla A E 2003 Role of the unperturbed limb and arms in the reactive recovery response to an unexpected slip during locomotion. $J$. Neurophysiol. 89 1727-37

[306] Chvatal S A and Ting L H 2012 Voluntary and Reactive Recruitment of Locomotor Muscle Synergies during Perturbed Walking J. Neurosci. 32 12237-50

[307] Sawers A, Pai Y-C C, Bhatt T and Ting L H 2017 Neuromuscular responses differ between slip-induced falls and recoveries in older adults. J. Neurophysiol. 117 509-22

[308] Berger W, Dietz V and Quintern J 1984 Corrective reactions to stumbling in man: neuronal co-ordination of bilateral leg muscle activity during gait. J. Physiol. 357 10925

[309] Dietz V, Quintern J and Berger W 1984 Corrective reactions to stumbling in man: Functional significance of spinal and transcortical reflexes Neurosci. Lett. 44 131-5

[310] Dietz V, Quintern J and Sillem M 1987 Stumbling reactions in man: significance of 
proprioceptive and pre-programmed mechanisms. J. Physiol. 386 149-63

[311] Dietz V, Horstmann G a, Berger W and W. B 1989 Interlimb coordination of leg-muscle activation during perturbation of stance in humans. J. Neurophysiol. 62(3) 680-93

[312] Stevenson A J T, Geertsen S S, Sinkjær T, Nielsen J B and Mrachacz-Kersting N 2015 Interlimb communication following unexpected changes in treadmill velocity during human walking J. Neurophysiol. 113 3151-8

[313] Troy K L and Grabiner M D 2006 Recovery responses to surrogate slipping tasks differ from responses to actual slips Gait Posture 24 441-7

[314] Janshen L, Santuz A, Ekizos A and Arampatzis A 2017 Modular control during incline and level walking in humans J. Exp. Biol. 220 jeb.148957

[315] Martino G, Ivanenko Y P, d'Avella A, Serrao M, Ranavolo A, Draicchio F, Cappellini G, Casali C and Lacquaniti F 2015 Neuromuscular adjustments of gait associated with unstable conditions J. Neurophysiol. 114 jn.00029.2015

[316] Aoi S, Tanaka T, Fujiki S, Funato T, Senda K and Tsuchiya K 2016 Advantage of straight walk instability in turning maneuver of multilegged locomotion: A robotics approach Sci. Rep. 630199

[317] De Groote F, Jonkers I and Duysens J 2014 Task constraints and minimization of muscle effort result in a small number of muscle synergies during gait Front. Comput. Neurosci. 8115

[318] Lacquaniti F, Ivanenko Y P and Zago M 2016 Are we ready to move beyond the reductionist approach of classical synergy control? Phys. Life Rev. 17 38-9

[319] Tang P F and Woollacott M H 1999 Phase-dependent modulation of proximal and distal postural responses to slips in young and older adults. J. Gerontol. A. Biol. Sci. Med. Sci. 54 M89-102

[320] $\mathrm{Yu} \mathrm{W}$ and Ikemoto Y 2007 An artificial reflex improves the perturbation-resistance of a human walking simulator Med. Biol. Eng. Comput. 45 1095-104

[321] Hill J and Fahimi F 2015 Active disturbance rejection for walking bipedal robots using the acceleration of the upper limbs Robotica 33 264-81

[322] Ikemoto Y and Yu W 2007 The roles of CPG phase modulation and reflexive muscular patterns in balance recovery reflexive responses to perturbation during walking - A simulation study Annual International Conference of the IEEE Engineering in Medicine and Biology - Proceedings (IEEE) pp 2389-92

[323] Ikemoto Y, Yu W and Inoue J 2008 A study on balance maintenance strategies during walking - A simulation study 2008 30th Annual International Conference of the IEEE Engineering in Medicine and Biology Society (IEEE) pp 5073-6

[324] Boone G N and Hodgins J K 1997 Slipping and tripping reflexes for bipedal robots Auton. Robots 4259

[325] Park J H and Kwon O 2001 Reflex control of biped robot locomotion on a slippery surface IEEE International Conference on Robotics and Automation vol 4 (IEEE) pp 4134-9

[326] Kajita S, Kaneko K, Harada K, Kaniehiro F, Fujiwara K, Hirukawa H, Kanehiro F, Fujiwara K, Hirukawa H, Kaniehiro F, Fujiwara K and Hirukawa H 2004 Biped walking on a low friction floor vol 4 (IEEE) pp 3546-52 
[327] Park J H and Cho H C 2000 An online trajectory modifier for the base link of biped robots to enhance locomotion stability 2000 ICRA Millennium Conference. IEEE International Conference on Robotics and Automation vol 4 (IEEE) pp 3353-8

[328] Hirose S 1984 A Study of Design and Control of a Quadruped Walking Vehicle Int. J. Rob. Res. 3 113-33

[329] Bekey G and Tomovic R 1986 Robot control by reflex actions Proc. 1986 IEEE Int. Conf. Robot. Autom. 3 240-7

[330] Mitobe K, Capi G and Nasu Y 2004 A new control method for walking robots based on angular momentum Mechatronics 14 163-74

[331] Prahlad V, Dip G and Meng-Hwee C 2008 Disturbance rejection by online ZMP compensation Robotica 26 9-17

[332] Yamaguchi J, Soga E, Set S A, Aoyagi D, Nagamatsu A and Takanishi A 1998 Development of a bipedal humanoid robot presupposing various whole body motions Adv. Robot. 13 297-9

[333] Hun-ok Lim, Kaneshima Y and Takanishi A 2002 Online walking pattern generation for biped humanoid robot with trunk Proceedings 2002 IEEE International Conference on Robotics and Automation (Cat. No.02CH37292) vol 3 (IEEE) pp 3111-6

[334] Zaoui C, Bruneau O, Ouezdou F B and Maalej A 2009 Simulations of the dynamic behavior of a bipedal robot with trunk and arms subjected to 3D external disturbances in a vertical posture, during walking and during object handling Multibody Syst. Dyn. 21 261-80

[335] Yang F, Anderson F C and Pai Y C 2008 Predicted threshold against backward balance loss following a slip in gait J. Biomech. 41 1823-31

[336] Oude Nijhuis L B, Allum J H J, Valls-Sole J, Overeem S and Bloem B R 2010 First Trial Postural Reactions to Unexpected Balance Disturbances: A Comparison With the Acoustic Startle Reaction J. Neurophysiol. 104 2704-12

[337] Keshner E A, Allum J H J and Pfaltz C R 1987 Postural coactivation and adaptation in the sway stabilizing responses of normals and patients with bilateral vestibular deficit Exp. Brain Res. 69 77-92

[338] Commissaris D A C M, Nieuwenhuijzen P H J A, Overeem S, De Vos A, Duysens J E J and Bloem B R 2002 Dynamic posturography using a new movable multidirectional platform driven by gravity J. Neurosci. Methods 113 73-84

[339] Bancroft M J and Day B L 2016 The Throw-and-Catch Model of Human Gait: Evidence from Coupling of Pre-Step Postural Activity and Step Location Front. Hum. Neurosci. 10635

[340] Potocanac Z, Pijnappels M, Verschueren S, van Dieën J and Duysens J 2016 Two-stage muscle activity responses in decisions about leg movement adjustments during trip recovery J. Neurophysiol. $115143-56$

[341] Hof A L and Duysens J 2018 Responses of human ankle muscles to mediolateral balance perturbations during walking Hum. Mov. Sci. 57 69-82

[342] Aoi S, Ogihara N, Funato T, Sugimoto Y and Tsuchiya K 2010 Evaluating functional roles of phase resetting in generation of adaptive human bipedal walking with a physiologically based model of the spinal pattern generator Biol. Cybern. 102 373-87 
[343] Pater M L, Rosenblatt N J and Grabiner M D 2015 Expectation of an upcoming large postural perturbation influences the recovery stepping response and outcome Gait Posture 41 335-7

[344] Pijnappels M, Bobbert M F and Van Dieën J H 2005 Control of support limb muscles in recovery after tripping in young and older subjects Exp. Brain Res. $160326-33$

[345] Troy K L and Grabiner M D 2005 The presence of an obstacle influences the stepping response during induced trips and surrogate tasks Exp. Brain Res. $161343-50$

[346] Visser J E, Oude Nijhuis L B, Janssen L, Bastiaanse C M, Borm G F, Duysens J and Bloem B R 2010 Dynamic posturography in Parkinson's disease: diagnostic utility of the "first trial effect" Neuroscience $168387-94$

[347] Hurt C P, Rosenblatt N J and Grabiner M D 2011 Form of the compensatory stepping response to repeated laterally directed postural disturbances Exp. Brain Res. 214 557-66

[348] Pijnappels M, Bobbert M F and Van Dieën J H 2006 EMG modulation in anticipation of a possible trip during walking in young and older adults J. Electromyogr. Kinesiol. 16 $137-43$

[349] Heiden T L, Sanderson D J, Inglis J T and Siegmund G P 2006 Adaptations to normal human gait on potentially slippery surfaces: The effects of awareness and prior slip experience Gait Posture 24 237-46

[350] Weerdesteyn V, Rijken H, Geurts A C H, Smits-Engelsman B C M, Mulder T and Duysens J 2006 A five-week exercise program can reduce falls and improve obstacle avoidance in the elderly Gerontology 52 131-41

[351] Groen B E, Smulders E, De Kam D, Duysens J and Weerdesteyn V 2010 Martial arts fall training to prevent hip fractures in the elderly Osteoporos. Int. 21 215-21

[352] Fujiwara K, Kajita S, Harada K, Kaneko K, Morisawa M, Kanehiro F, Nakaoka S and Hirukawa H 2006 Towards an optimal falling motion for a humanoid robot Proceedings of the 2006 6th IEEE-RAS International Conference on Humanoid Robots, HUMANOIDS (IEEE) pp 524-9

[353] Ogata K, Terada K T K and Kuniyoshi Y K Y 2007 Falling motion control for humanoid robots while walking 2007 7th IEEE-RAS International Conference on Humanoid Robots (IEEE) pp 306-11

[354] Wang J, Whitman E and Stilman M 2012 Whole-body trajectory optimization for humanoid falling ... Control Conference (ACC), ... (IEEE) pp 4837-42

[355] Ma G, Huang Q, Yu Z, Chen X, Hashimoto K, Takanishi A and Liu Y H 2015 Bioinspired falling motion control for a biped humanoid robot IEEE-RAS International Conference on Humanoid Robots vol 2015-Febru (IEEE) pp 850-5

[356] Dietz V, Zijlstra W and Duysens J 1994 Human neuronal interlimb coordination during split-belt locomotion Exp. Brain Res. 101 513-20

[357] Prokop T, Berger W, Zijlstra W and Dietz V 1995 Adaptational and learning processes during human split-belt locomotion: interaction between central mechanisms and afferent input. Exp. Brain Res. 106 449-56

[358] Reisman D S 2005 Interlimb Coordination During Locomotion: What Can be Adapted and Stored? J. Neurophysiol. 94 2403-15

[359] Choi J T and Bastian A J 2007 Adaptation reveals independent control networks for 
human walking Nat. Neurosci. 10 1055-62

[360] Hoogkamer W, Bruijn S M, Potocanac Z, Van Calenbergh F, Swinnen S P and Duysens J 2015 Gait asymmetry during early split-belt walking is related to perception of belt speed difference J. Neurophysiol. 114 1705-12

[361] Hoogkamer W, Bruijn S M, Sunaert S, Swinnen S P, Van Calenbergh F and Duysens J 2015 Adaptation and aftereffects of split-belt walking in cerebellar lesion patients. $J$. Neurophysiol. 114 1693-704

[362] Hoogkamer W and O'Brien M K 2016 Sensorimotor recalibration during split-belt walking: task-specific and multisensory? J. Neurophysiol. 116 1539-41

[363] Statton M A, Vazquez A, Morton S M, Vasudevan E V L and Bastian A J 2018 Making Sense of Cerebellar Contributions to Perceptual and Motor Adaptation Cerebellum 17 $111-21$

[364] Bastian A J 2008 Understanding sensorimotor adaptation and learning for rehabilitation Curr. Opin. Neurol. 21 628-33

[365] Morton S M and Bastian A J 2006 Cerebellar Contributions to Locomotor Adaptations during Splitbelt Treadmill Walking J. Neurosci. 26 9107-16

[366] Fujiki S, Aoi S, Funato T, Tomita N, Senda K and Tsuchiya K 2015 Adaptation mechanism of interlimb coordination in human split-belt treadmill walking through learning of foot contact timing: a robotics study. J. R. Soc. Interface 120542

[367] Meyns P, Bruijn S M and Duysens J 2013 The how and why of arm swing during human walking Gait Posture 38 555-62

[368] Chyou T, Liddell G F and Paulin M G 2011 An upper-body can improve the stability and efficiency of passive dynamic walking J. Theor. Biol. 285 126-35

[369] Hooper S L and Büschges A Neurobiology of motor control : fundamental concepts and new directions 